\title{
LA-8641-MS
}

\section{Transportation Energy Flows for 1976}

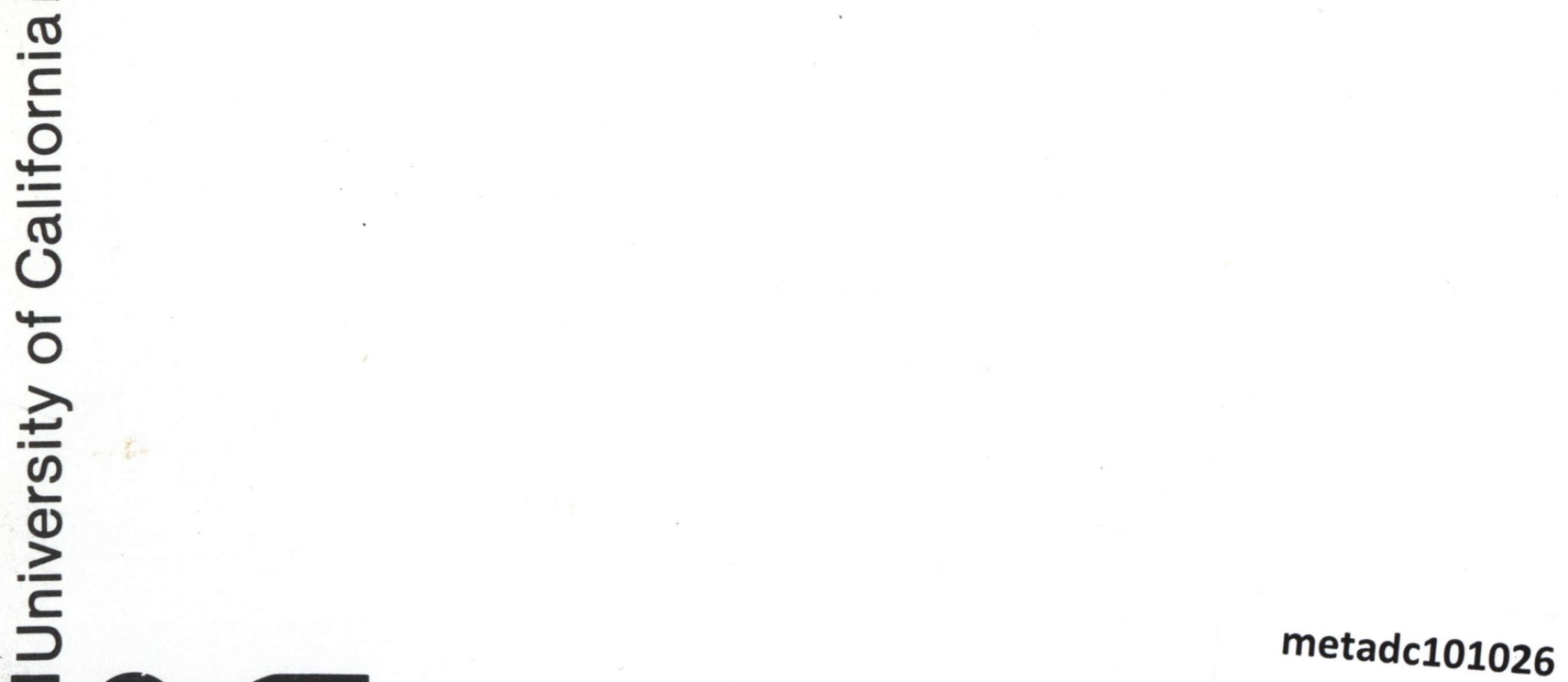


An Affirmative Action/Equal Opportunity Employer

This report was not edited by the Technical Information staff.

This work was supported by the US Department of Energy, Office of Conservation and Solar Programs and Office of Transportation Programs. ment. Neither the United States Government nor any agency thereof, nor any of their employees, makes any warranty, express or implied, or assumes any legal liability or responsibility for the accuracy, completeness, or usefulness of any information, apparatus, product, or process disclosed, or represents that its use would not infringe privately owned rights. Reference herein to any specific commercial product, process, or service by trade name, trademark, manufacturer, or otherwise, does not necessarily constitute or imply its endorsement, recommendation, or favoring by the United States Government or any agency thereof. The views and opinions of authors expressed herein do not necessarily state or reflect those of the United States Government or any agency thereof. 
UC-95c

Issued: December 1980

\title{
Transportation Energy Flows for 1976
}

\author{
R. B. Kidman
}

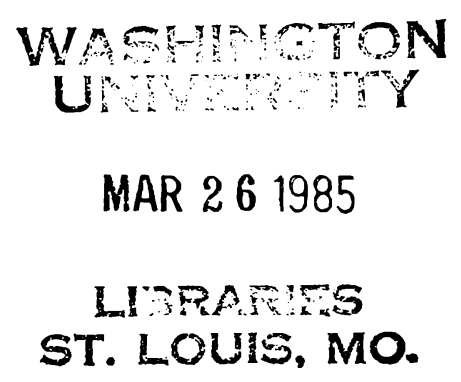



TRANSPORTATION ENERGY FLOWS FOR 1976

by

R. B. Kidman

ABSTRACT

Highly graphic, condensed, and informative illustrations of the 1976 transportation energy flows are presented for each of the 50 states, the 10 US Federal Standard Regions, the Mi1itary, and the United States. Each diagram shows the energy flow from primary energies, to fuel types, to the modes of transportation, plus the pollution emissions produced by each mode. The diagrams are an initial attempt to provide a convenient overview and standardized framework of the transportation system.

\section{INTRODUCTION}

Graphical displays can be a popular aid in understanding complex systems. This was the case when we produced ${ }^{l}$ simplified diagrams of the total energy flow in each state. Judging from the numerous requests, the convenient overview and standardized framework of the diagrams found wide acceptance.

Such graphics for each state would be a prohibitive manual drafting effort. However, as computer graphics improve not only are such tasks possible, we can begin to think about much more complex 11lustrations that tell better and more complete stories.

For these reasons, the Department of Energy's (DOE) Office of Transportation Programs (CS/OTP) has asked us to explore the possibility of generating similar diagrams that focus on the transportation system. From these inftial

Assistance for this project was provided by P. Patterson and D. Maxfield of DOE/ CS/OTP, 1000 Ind ependence Avenue, S.W., Washington, D.C. 20585 and F. Westbrook of TERA, 2849 Meadow View Road, Falls Church, VA 22042. 
diagrams, which we present in this document, we hope to evolve more advanced transportation system graphics. If the reader has questions or ideas for improvements, please call the author at 505/667-7671 (commercial) or 843-7671 (FTS).

\section{DIAGRAM DESCRIPTION}

Each transportation energy flow diagram displays the flow of energy from the primary energy sources, through the various fuels, to the modes of transportation. The pollution produced by each mode is shown by change-of-scale flow lines leading to the residuals [carbon monoxide ( $\mathrm{CO}$ ), nitrogen oxides (NOX), and hydrocarbons (HC)].

The widths of the energy flow lines are proportional to the amount of flow through each path. The energy flow lines are labeled with their percentage of the total flows from the box where they originate. Each energy box is labeled with a name and the amount of energy in units of trillions of British Thermal Units (TBTU). The energy depicted in the diagrams refers only to the energy ultimately consumed by the various transportation modes. That is, no indirect energies or energy losses are carried along.

The widths of the pollution flow lines vary to accomodate the change of scale required to represent this different quantity. As these flow lines leave a box in the transportation modes column, their widths are proportional to the residuals produced in that box. As these flow lines enter the residual column, their widths are proportional to the amount of pollutant flowing through each path. The pollution flow lines and boxes are labeled with the amount of residuals in units of thousands of tons.

The scale of every diagram is automatically adjusted so that each state's flows occupy the same size physical space on a page. This allows a more accurate visual comparison of the flows within a state.

Flow lines or boxes whose magnitudes are less than 0.005 are not shown. The number of decimal places accompanying each number is not a statement of accuracy but merely an arbitrary presentation choice. When an energy flow line has no percentage number, it simply means that $100 \%$ of the left-hand box is flowing through the path. 


\section{ENERGY DATA}

As much as possible, data from the End Use Energy Consumption Data Base ${ }^{2}$ (ECDB) has been used to construct the diagrams. The ECDB is a definitive accounting of U.S. transportation energy use compiled by Oak Ridge National Laboratory* for the Energy Information Administration of DOE. It contains the methods and results of estimating transportation fuel and energy use for a multitude of categories within all sectors of the economy for each of the ten years $1967-1976$.

The procedures used to compute the diagrams are described below. As will be seen, several modes (gasoline, LPG, and electricity to transit buses; diesel to intercity buses; diesel to recreational boats; and electricity to liquids pipelines) had only a national number and had to be given a state-by-state breakdown. Several other modes (gasoline and diesel to federal trucks; LPG to trucks; and diesel, natural gas, and electricity to natural gas pipelines) had to be derived by disaggregating lumped fuel numbers into various fuels.

In the fuel-mode descriptions that follow, you will find many instances of numbers appearing in parentheses. This is the author's method of specifically identifying the ECDB table that contains the mentioned data. For example, ... motorcycles (8.1) ... means that the motorcycle data of interest can be found in table 8.1 of the ECDB.

Gasoline to Highway Passenger - This flow is the gasoline consumed by motorcycles ( 8.1 ), school buses (6.2), federal buses (6.3), commercial autos (5.2), federal civilian autos (5.4), household autos (5.5), taxicabs (5.3), and transit buses (6.6), plus the LPG consumed by transit buses (6.5). The transit bus gasoline and LPG consumption was allocated among the states according to the state breakdown of transit bus diesel fuel consumption (6.6).

Gasoline to Trucks - This flow is the gasoline consumed by intercity nongovernment trucks (7.9), local non-government trucks (7.6), and federal trucks, plus the LPG consumed by trucks. The gasoline consumed by federal trucks was assumed to be $80.3 \%$ (as suggested by Method 336 in ECDB) of the fuel consumed by federal trucks (7.12). The LPG consumed by trucks was assumed to be the total local truck energy use minus the total local truck gasoline and diesel fuel use $(7.4+7.5-7.6-7.7)$.

\footnotetext{
${ }^{\star}$ End Use Energy Consumption Data Base: Transportation Sector
} 
Gasoline to Air - This flow is aviation gasoline consumed by aircraft (3.11).

Gasoline to Marine - This flow is the gasoline consumed by recreational boats $(9.2)$.

Diesel to Highway Passenger - This flow is the diesel fuel consumed by transit buses (6.6) and intercity buses (6.4). The intercity bus contribution was given the same state-wise break down as intercity truck fuel, (7.8 and 7.9).

Diesel to Trucks - This flow is the diesel fuel consumed by intercity nongovernment trucks (7.8), local non-government trucks (7.7), and federal trucks. The federal truck contribution was assumed to be $19.7 \%$ (as suggested by Method 336 in ECDB) of the fuel consumed by federal trucks (7.12).

Diesel to Marine - This flow is the diesel fuel consumed by commercial vessels (9.3) and recreational boats (9.1). The recreational boat contribution was given the same state-wise breakdown as recreational boat gasoline consumption $(9.2)$.

Diesel to Pipeline - This flow is the energy consumed by steam-powered pipeline pumps. The energy was assumed to be provided by diesel fuel and the state-wise values were obtained by multiplying the total energy consumed by natural gas pipelines $(10.2)$ by the factor $0.008 / 1.023$ (as suggested by Method 510 in $\mathrm{E}(\mathrm{DB})$.

Diesel to Rail- This flow is the diesel fuel consumed by commuter rail (4.3), intercity passenger rail (4.4), and freight rail (4.5).

Jet to Air - This flow is the jet fuel consumed by aircraft (3.11).

Residual to Marine - This flow is the residual fuel consumed by commercial vessels (9.3).

Residual to Rail - This flow is the residual fuel consumed by freight rail (4.5).

Natural Gas to Pipeline - This flow was obtained by multiplying the total energy consumed by natural gas pipelines (10.2) by the factor $1 / 1.023$ (as suggested by Method 510 in ECDB).

Electricity to Highway Passenger - This flow is the electricity used by transit buses (6.5), which was broken down state-wise in the same fashion as transit bus diesel fuel use $(6.6)$.

Electricity to Pipeline - This flow is the electricity used by natural gas and liquids pipelines. The electricity used by natural gas pipelines was obtained by multiplying the total energy consumed by natural gas pipelines (10.2) 
by the factor $0.015 / 1.023$ (as suggested by Method 510 in ECDB). The electricity used by liquids pipelines (crude o11, oil products, and water) (10.3) was a110cated to the states in the same way the gas pipeline energy was allocated (10.2). Arizona's coal slurry pipeline electricity use of $1.076 \mathrm{TBTU}^{3}$ was also added to this flow.

Electricity to Rail - This flow is the electricity used by transit rail (4.2), commuter rail (4.3), and intercity passenger rail (4.4).

0i1, Nat. Gas, Hydro, Nuclear, and Coal to Electricity - The amounts of oil, natural gas, hydro, nuclear, and coal energies converted to electricity for transportation consumption were obtained for each state by assuming their percentages were the same as the percentages that fed the total 1975 electricity generation in each state. ${ }^{1}$

Imported - The percentage of each state's transportation petroleum that was imported was assumed to be the same as each state's 1975 Imported percentage for all petroleum. ${ }^{1}$

Military - The military consumption of jet (11.3), gasoline (11.2) and diesel (11.2), and residual (11.2) fuels were assumed to be used by the air, land, and sea modes, respectively. There is no data avallable for the nuclear energy used by the U.S. Navy.

Population - Our source for population data is the Bureau of the Census. ${ }^{4}$ The MBTU/CAPITA in the diagram legends refers to the average energy consumption per person in units of millions of BTU.

IV. POLLUTION DATA

Table I contains estimates of the residuals produced when a unit of energy is consumed by the various transportation modes. The total pollution emitted by any mode is simply its total energy multiplied by the appropriate emission factor. These emission factors were applied to the fuel-mode combinations shown in the first column. The last column identifies the 1977 data units of Ref. 5 from which the various emissions data were obtained. Sometimes the emission data from several units were averaged with their respective unit energies to provide weighted emission averages.

V. FEDERAL REGIONS

The U.S. diagram and the 10 regional diagrams were produced by adding the constituent state contributions for each component of the diagram. Please note 
that the Military is not included in the US diagram and that the District of Columbia is included with Maryland. The states comprising each region are as shown in Table II.

TABLE I

POLLUTION EMISSIONS

\begin{tabular}{|c|c|c|c|c|c|}
\hline Application & \multicolumn{3}{|c|}{ Emissions (Tons/TBTU) } & \multirow{2}{*}{$\begin{array}{l}\text { Data } \\
\text { Unit }\end{array}$} & \multirow{2}{*}{$\begin{array}{l}\text { Source } \\
\text { No. ID's }\end{array}$} \\
\hline Fue1s;Modes & NOX & $\mathrm{HC}$ & $\mathrm{CO}$ & & \\
\hline $\begin{array}{l}\text { Gasoline;Autos,Taxi : LPG;Buses } \\
\text { Gasoline,LPG;Trucks } \\
\text { Gasoline;Air } \\
\text { Gasoline;Marine } \\
\text { Diesel;Trucks,Pipelines }\end{array}$ & $\begin{array}{r}119.66 \\
463.75 \\
36.36 \\
26.61 \\
1413.22\end{array}$ & $\begin{array}{r}49.07 \\
762.26 \\
72.73 \\
4434.78 \\
141.32\end{array}$ & $\begin{array}{r}1076.93 \\
5191.90 \\
2181.82 \\
2695.65 \\
859.50\end{array}$ & & $\begin{array}{l}76,77 \\
72 \\
89 \\
85 \\
71\end{array}$ \\
\hline $\begin{array}{l}\text { Diese1;Rail } \\
\text { Diese1;Marine } \\
\text { Jet Fuel;Air } \\
\text { Residual;Marine, Ra11 } \\
\text { Nat. Gas; Pipelines }\end{array}$ & $\begin{array}{r}282.61 \\
1264.55 \\
10.42 \\
258.79 \\
29.19\end{array}$ & $\begin{array}{r}188.41 \\
83.33 \\
33.15 \\
42.21 \\
3.89\end{array}$ & $\begin{array}{r}263.77 \\
505.29 \\
31.06 \\
492.46 \\
9.73\end{array}$ & & $\begin{array}{l}73 \\
69 \\
74,75,82,83,90 \\
70 \\
61\end{array}$ \\
\hline $\begin{array}{l}\text { Diesel; Intercity Buses } \\
\text { Gasoline;Buses } \\
\text { Diesel; Transit Buses } \\
\text { Gasoline;Motorcycles } \\
\text { Diesel; Passenger Rail }\end{array}$ & $\begin{array}{r}1299.07 \\
225.00 \\
1295.65 \\
41.45 \\
229.31\end{array}$ & $\begin{array}{r}127.10 \\
385.42 \\
127.39 \\
6282.89 \\
152.76\end{array}$ & $\begin{array}{r}789.72 \\
3229.17 \\
768.96 \\
25986.84 \\
213.79\end{array}$ & & $\begin{array}{l}79 \\
80 \\
81 \\
84 \\
88\end{array}$ \\
\hline $\begin{array}{l}\text { Jet Fue1;Military Air } \\
\text { Gasoline;Military Land } \\
\text { Diesel;Military Land } \\
\text { Residual;Military Sea }\end{array}$ & $\begin{array}{r}8.57 \\
636.00 \\
1258.99 \\
223.33\end{array}$ & $\begin{array}{r}44.36 \\
1040.00 \\
83.45 \\
42.00\end{array}$ & $\begin{array}{r}41.35 \\
6880.00 \\
503.60 \\
493.33\end{array}$ & & $\begin{array}{l}93 \\
94 \\
95 \\
96\end{array}$ \\
\hline
\end{tabular}


TABLE II

FEDERAL REGIONS

\begin{tabular}{|c|c|c|c|c|}
\hline Region I & Region II & Region III & Region IV & Region V \\
\hline $\begin{array}{l}\text { Connecticut } \\
\text { Maine } \\
\text { Massachusetts } \\
\text { New Hampshire } \\
\text { Rhode Island } \\
\text { Vermont }\end{array}$ & $\begin{array}{l}\text { New Jersey } \\
\text { New York }\end{array}$ & $\begin{array}{l}\text { Delaware } \\
\text { Maryland + DC } \\
\text { Pennsylvania } \\
\text { Virginia } \\
\text { West Virginia }\end{array}$ & $\begin{array}{l}\text { Alabama } \\
\text { Florida } \\
\text { Georgia } \\
\text { Kentucky } \\
\text { Mississippi } \\
\text { North Carolina } \\
\text { South Carolina } \\
\text { Tennessee }\end{array}$ & $\begin{array}{l}\text { Illinois } \\
\text { Indiana } \\
\text { Michigan } \\
\text { Minnesota } \\
\text { Ohio } \\
\text { Wisconsin }\end{array}$ \\
\hline Region VI & Region VII & Region VIII & Region IX & Region $\mathrm{X}$ \\
\hline $\begin{array}{l}\text { Arkansas } \\
\text { Louisiana } \\
\text { New Mexico } \\
\text { Oklahoma } \\
\text { Texas }\end{array}$ & $\begin{array}{l}\text { Iowa } \\
\text { Kansas } \\
\text { Missour } 1 \\
\text { Nebraska }\end{array}$ & $\begin{array}{l}\text { Colorado } \\
\text { Montana } \\
\text { North Dakota } \\
\text { South Dakota } \\
\text { Utah } \\
\text { Wyoming }\end{array}$ & $\begin{array}{l}\text { Arizona } \\
\text { California } \\
\text { Hawail } \\
\text { Nevada }\end{array}$ & $\begin{array}{l}\text { Alaska } \\
\text { Idaho } \\
\text { Oregon } \\
\text { Washington }\end{array}$ \\
\hline
\end{tabular}

\section{ACKNOWLEDGMENTS}

We gratefully acknowledge Dolly M. McClellan for her assistance in reducing and manipulating the data.

\section{REFERENCE $S$}

1. R. B. Kidman, R. J. Barrett, and D. R. Koenig, "Energy Flow Patterns for 1975," Los Alamos Scientific Laboratory report LA-6770 (1977).

2. J. N. Hooker, A. B. Rose, and D. L. Greene, ORNL, "End Use Energy Consumption Data Base: Transportation Sector," U. S. Department of Energy report DOE-EIA-CR-7405-01 (1980).

3. W. F. Banks, "Energy Consumption in the Pipeline Industry," U. S. Department of Energy report SAN-1171-2 (1977).

4. U. S. Department of Commerce, "Population Estimates and Projections," Bureau of the Census, Series P-25, No. 876 (Feb. 1980).

5. Mitre Corporation for DOE ASEV Office of Environmental Assessments, "RESGEN Coefficients for the Strategic Environmental Assessment System" (June 1978). 


\section{TRANSPORTATION ENERGY FLOWS ALABAMA 1976}

UNITS

ALABAMA 1976

ENERGY = TRILLIONS OF BTU POLLUTION = THOUSANDS OF TONS

SOURCES

$\longrightarrow$

FUELS

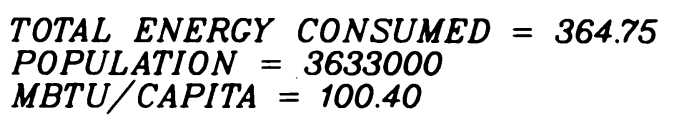

SOURCES

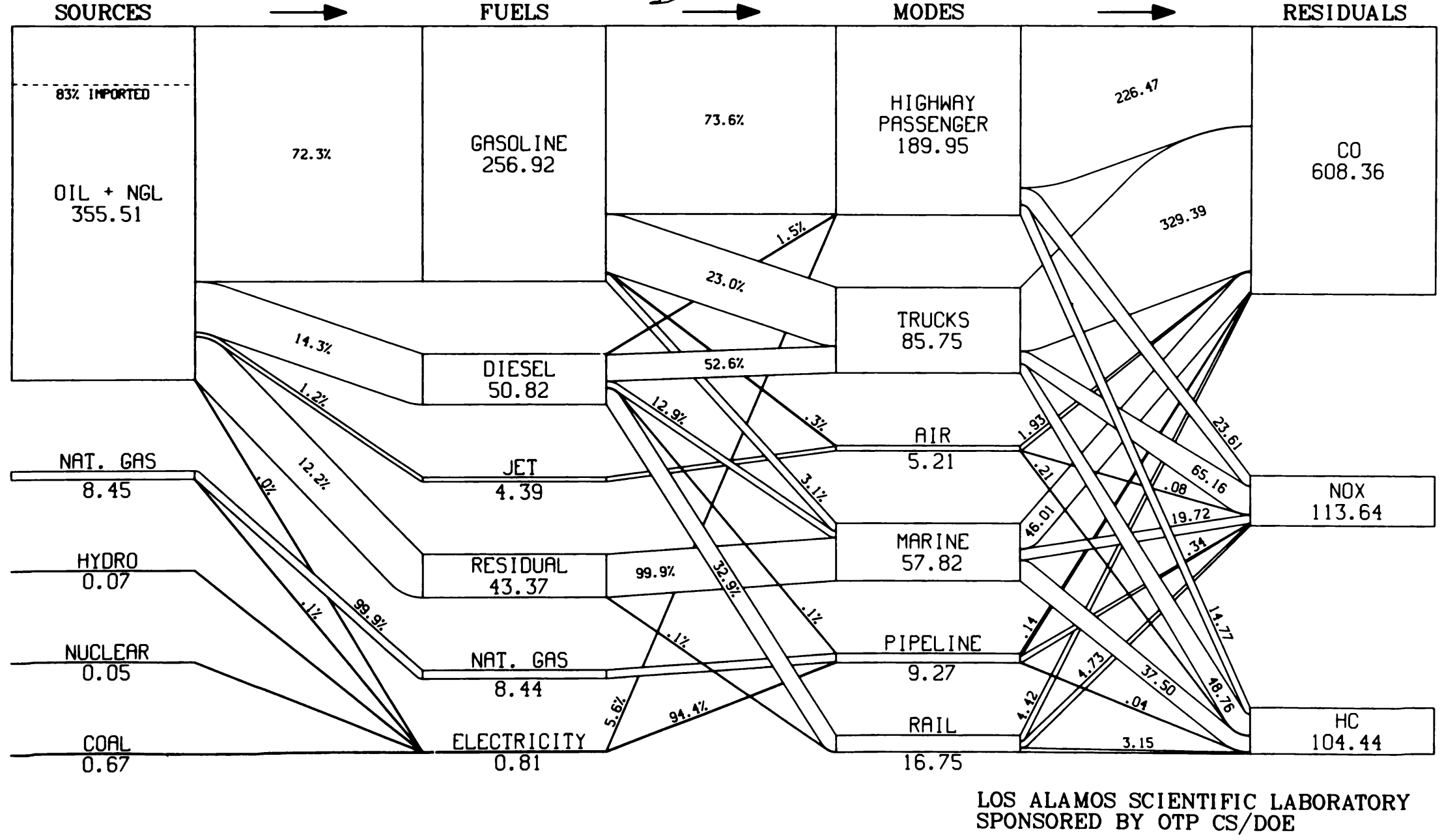

FUELS

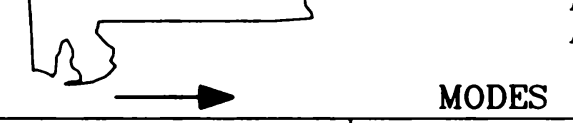

TOTAL ENERGY CONSUMED $=364.75$
POPULATION $=3633000$ $M B T U / C A P I T A=100.40$ 


\section{TRANSPORTATION ENERGY FLOWS ALASKA 1976}

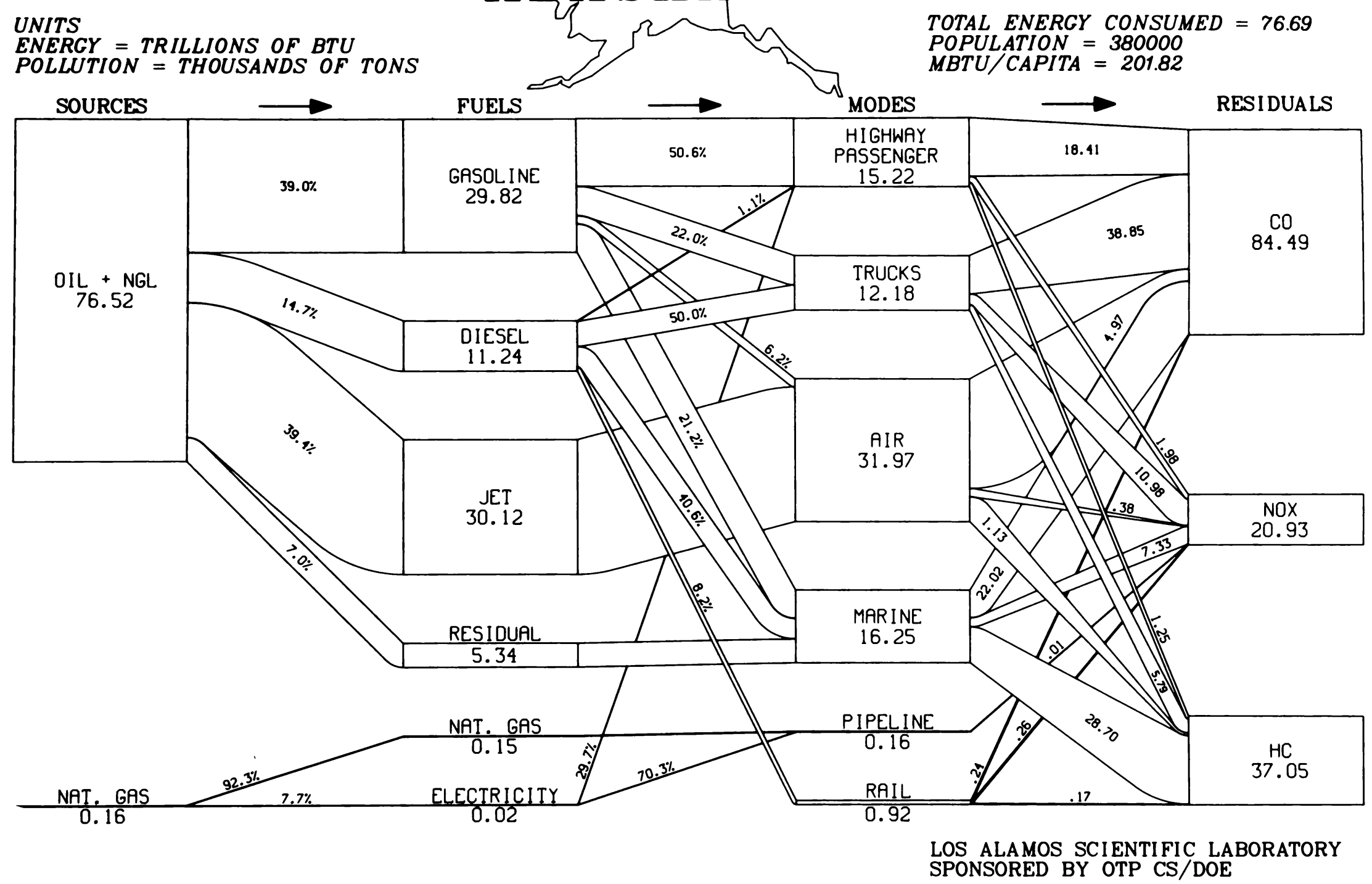




\section{TRANSPORTATION ENERGY FLOWS ARIZONA 1976}

\section{UNITS}

ENERCY = TRILLIONS OF BTU

POLLUTION = THOUSANDS OF TONS

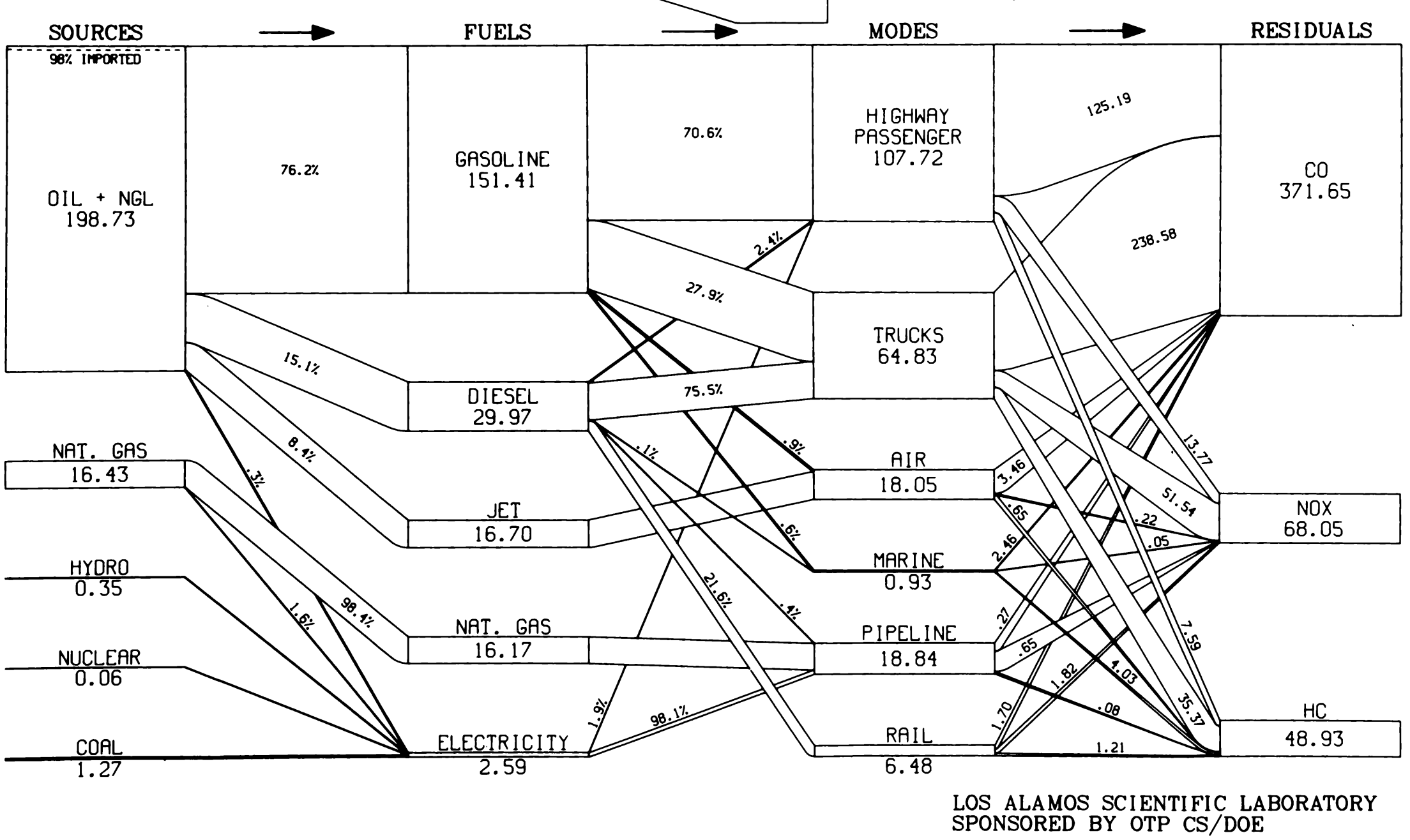

TOTAL ENERGY CONSUMED $=216.84$ POPULATION $=2217000$ MBTU $C A P I T A=97.81$ 


\section{TRANSPORTATION ENERGY FLOWS ARKANSAS 1976}

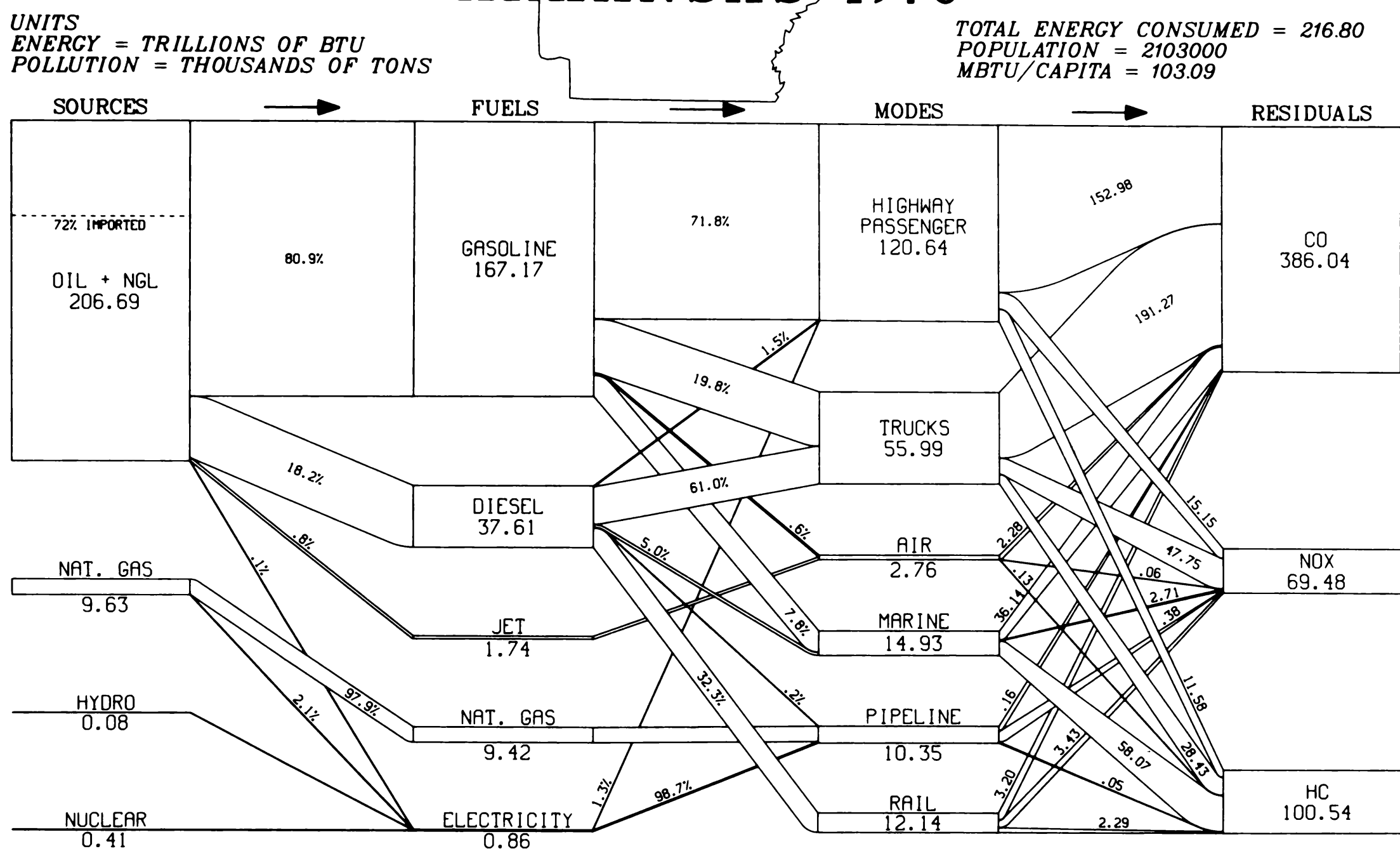

LOS ALAMOS SCIENTIFIC LABORATORY 


\section{TRANSPORTATION ENERGY FLOWS CALIFORNIA 1976}

UNITS

ENERGY = TRILLIONS OF BTU

POLLUTION $=$ THOUSANDS OF TONS

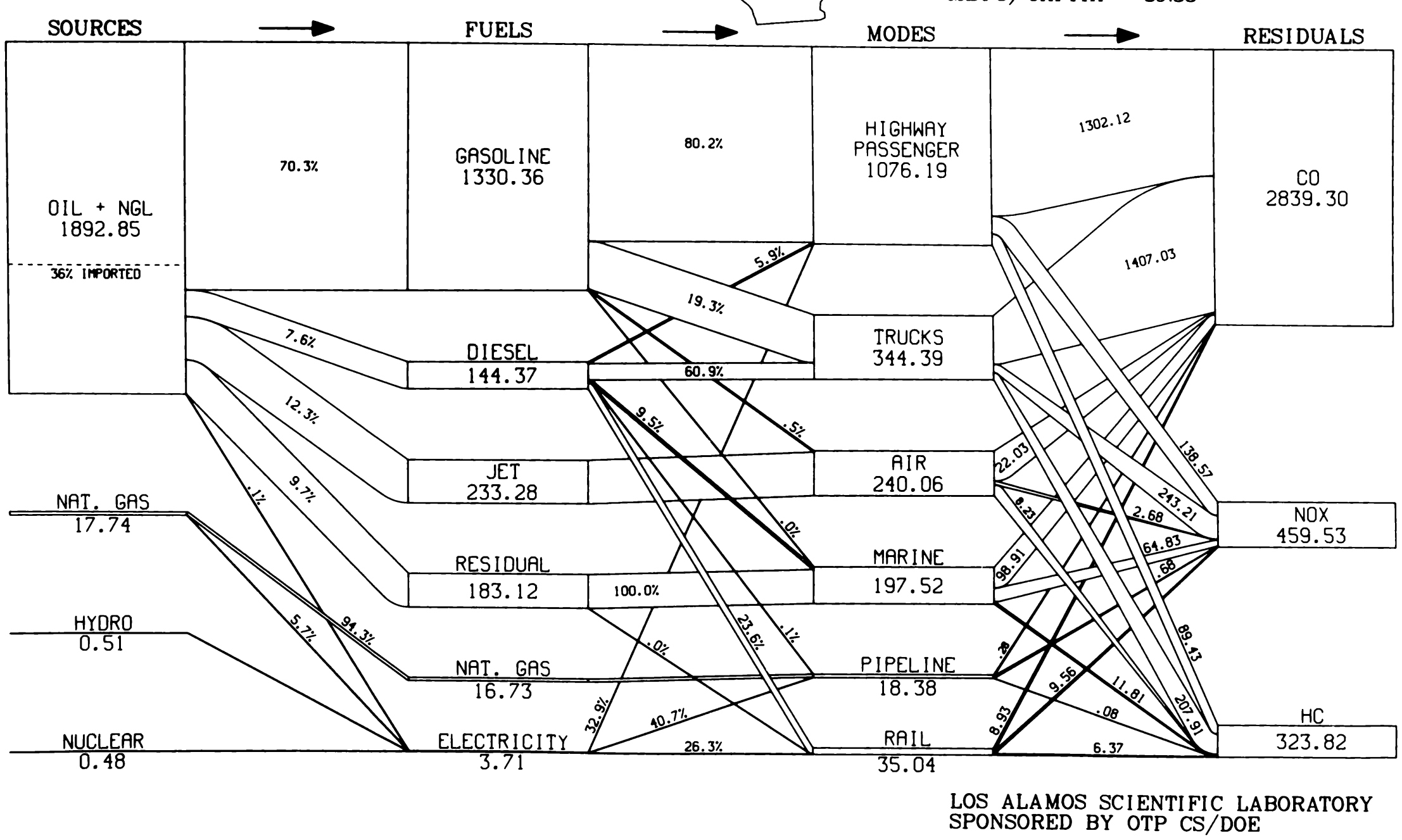

TOTAL ENERGY CONSUMED = 1911.61 POPULATION $=21267000$ MBTU $/$ CAPITA $=89.88$ SPONSORED BY OTP CS/DOE 


\section{TRANSPORTATION ENERGY FLOWS COLORADO 1976}

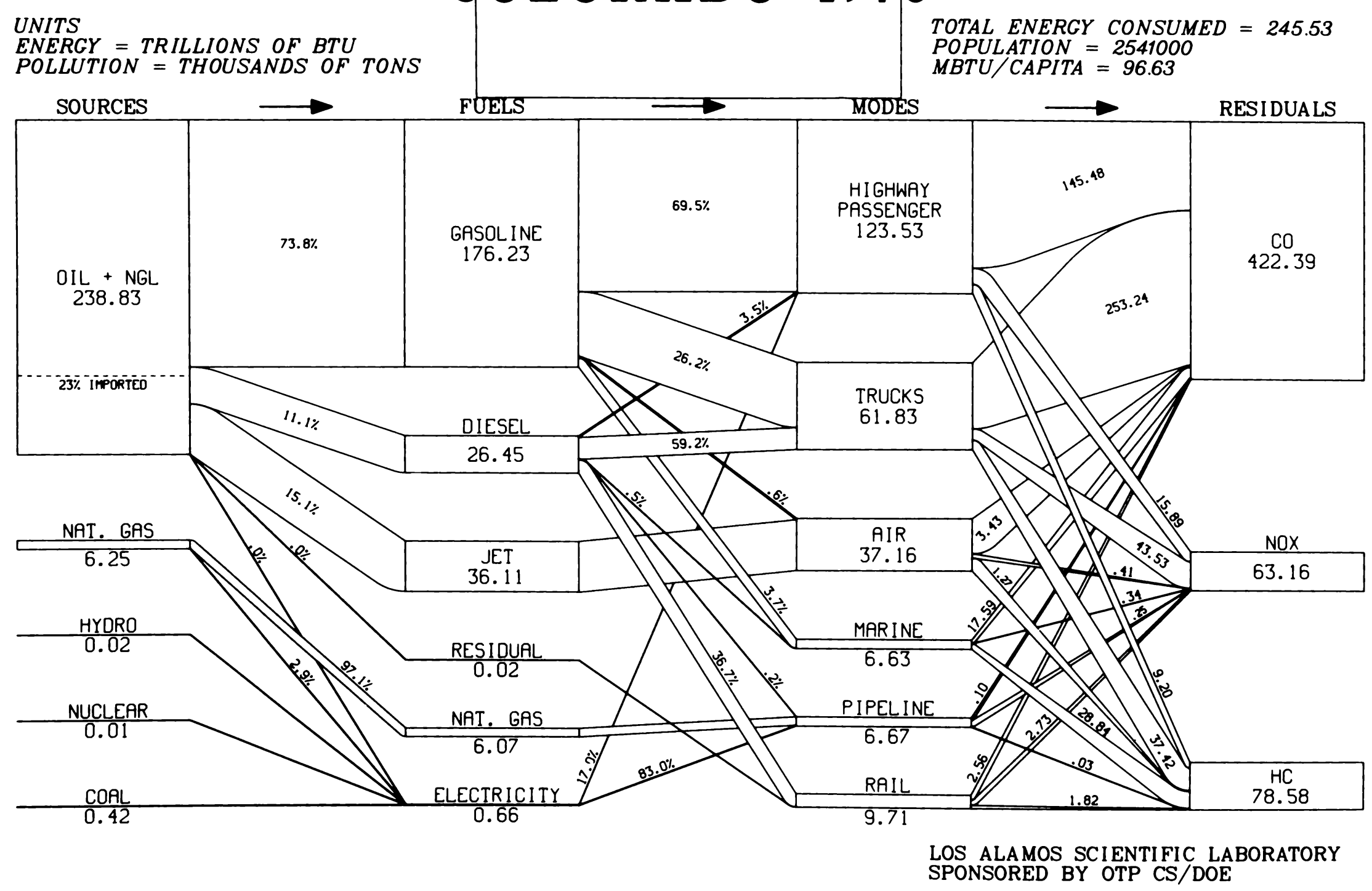




\section{TRANSPORTATION ENERGY FLOWS CONNECTICUT 1976}

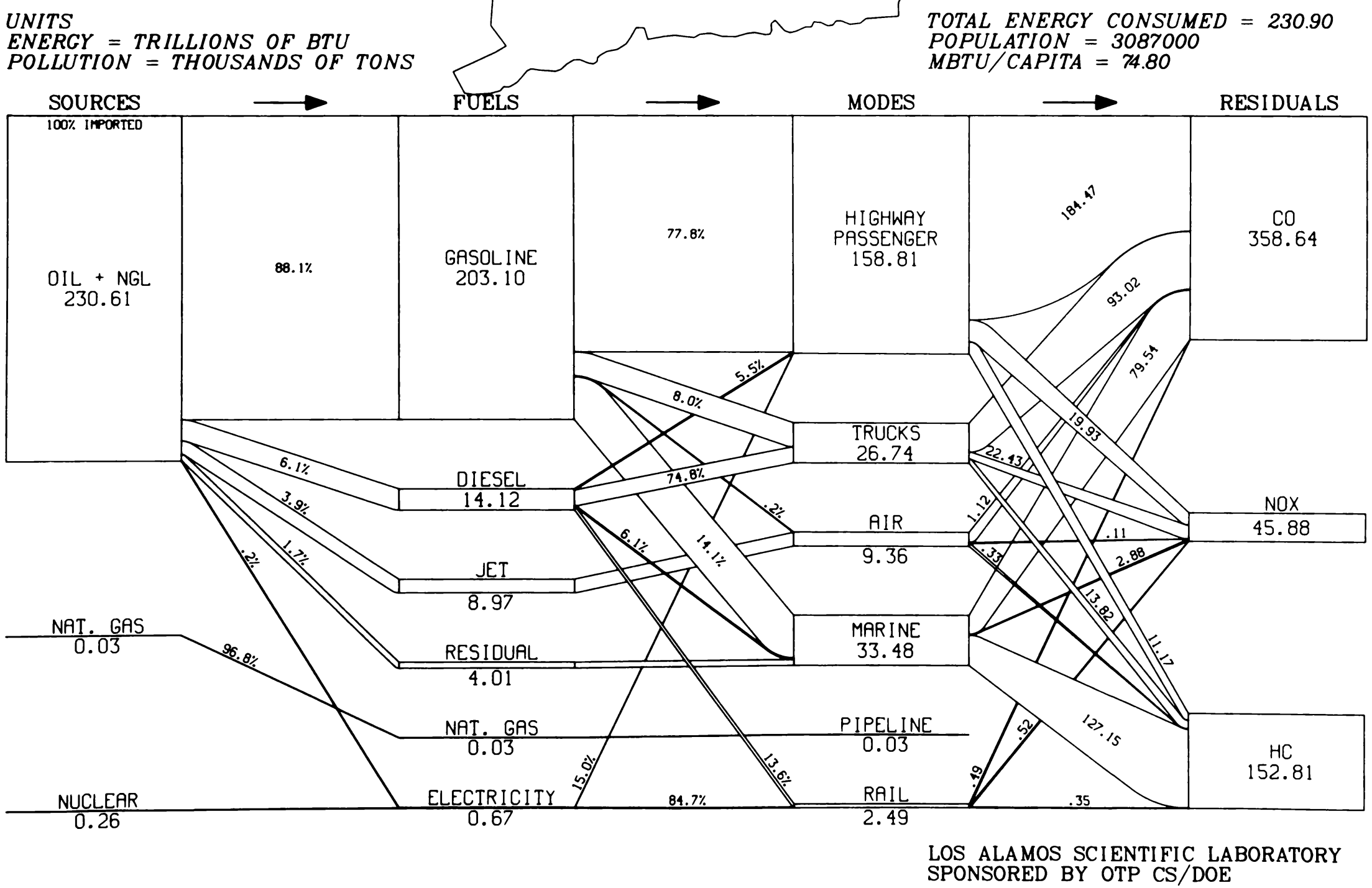




\section{TRANSPORTATION ENERGY FLOWS DELAWARE 1976}

\section{UNITS}

ENERCY = TRILLIONS OF BTU

POLLUTION = THOUSANDS OF TONS

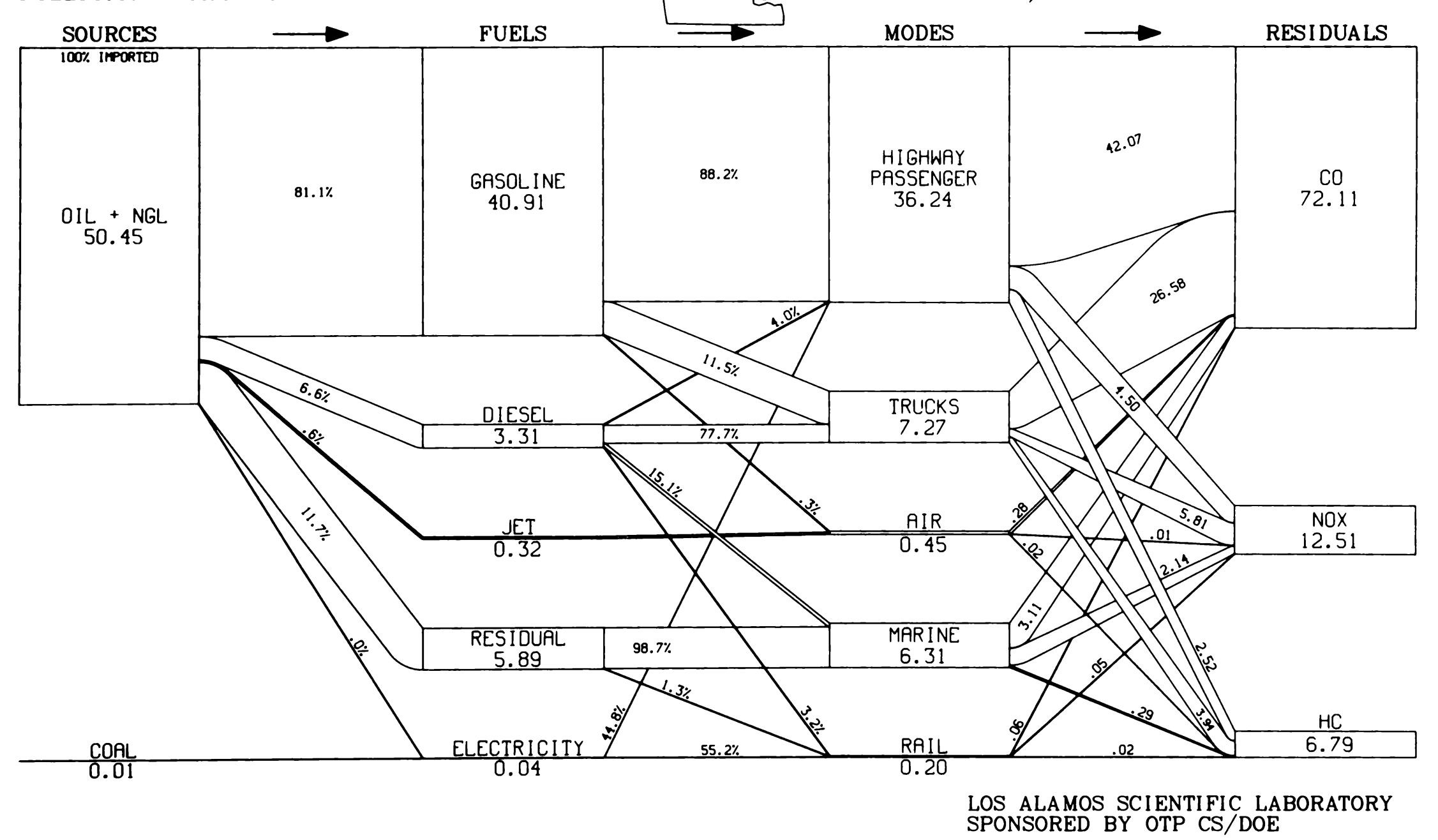

TOTAL ENERGY CONSUMED $=50.47$ POPULATION $=576000$ SPONSORED BY OTP CS/DOE 


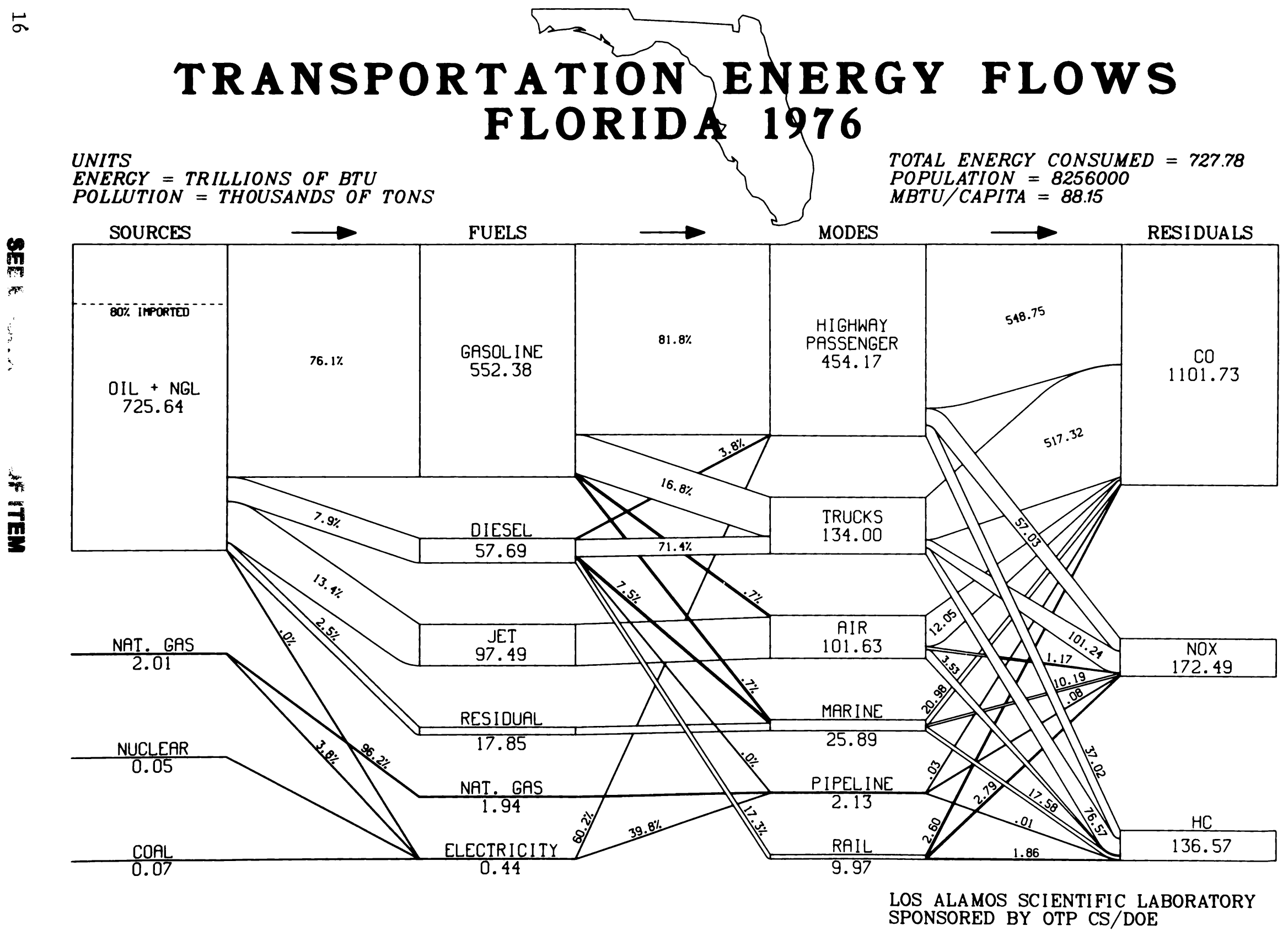




\section{TRANSPORTATION ENERGY FLOWS IDAHO 1976}

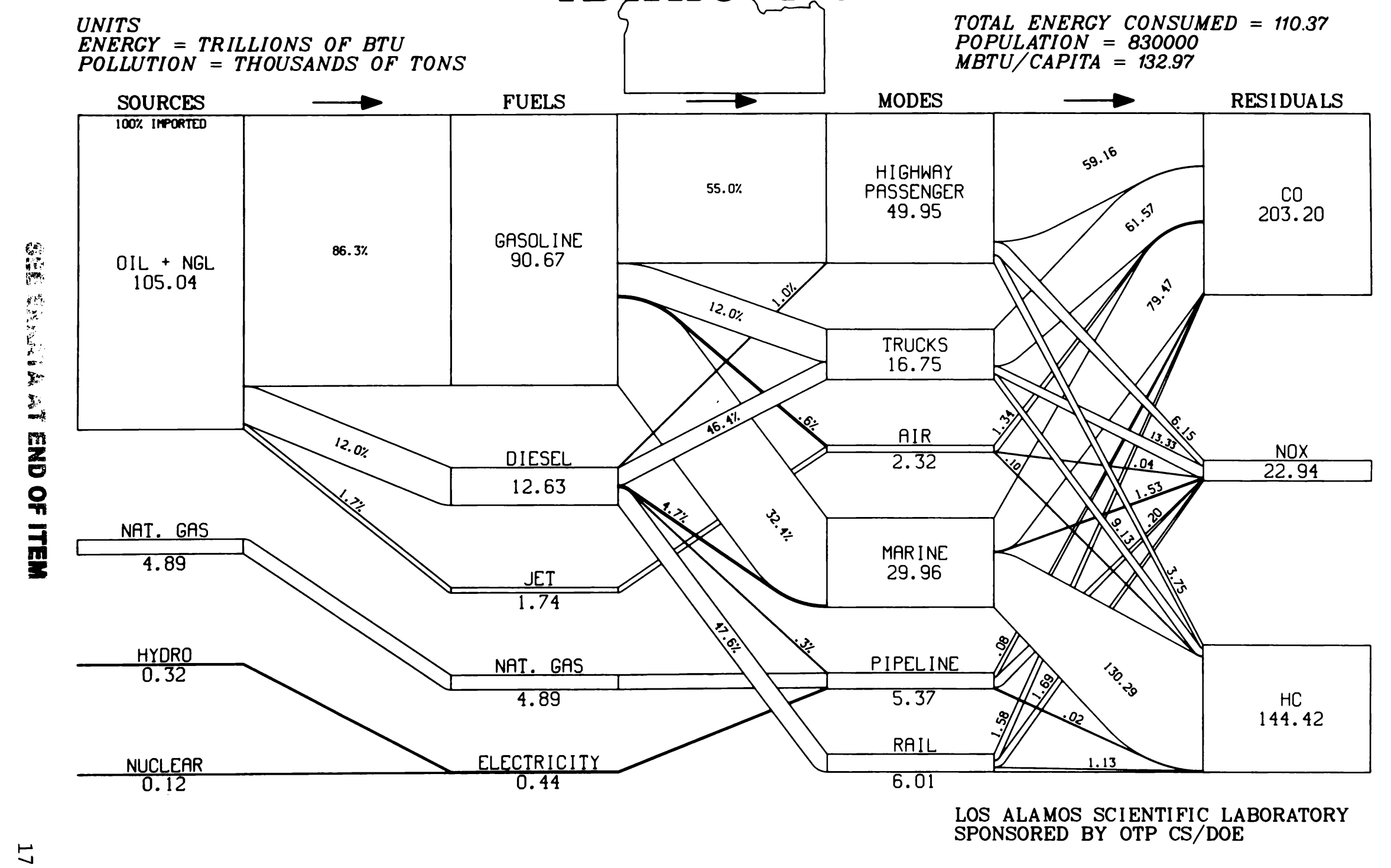




\section{TRANSPORTATION ENERGY FLOWS ILLINOIS 1976}

\section{UNITS}

ENERGY = TRILLIONS OF BTU

POLLUTION = THOUSANDS OF TONS

SOURCES

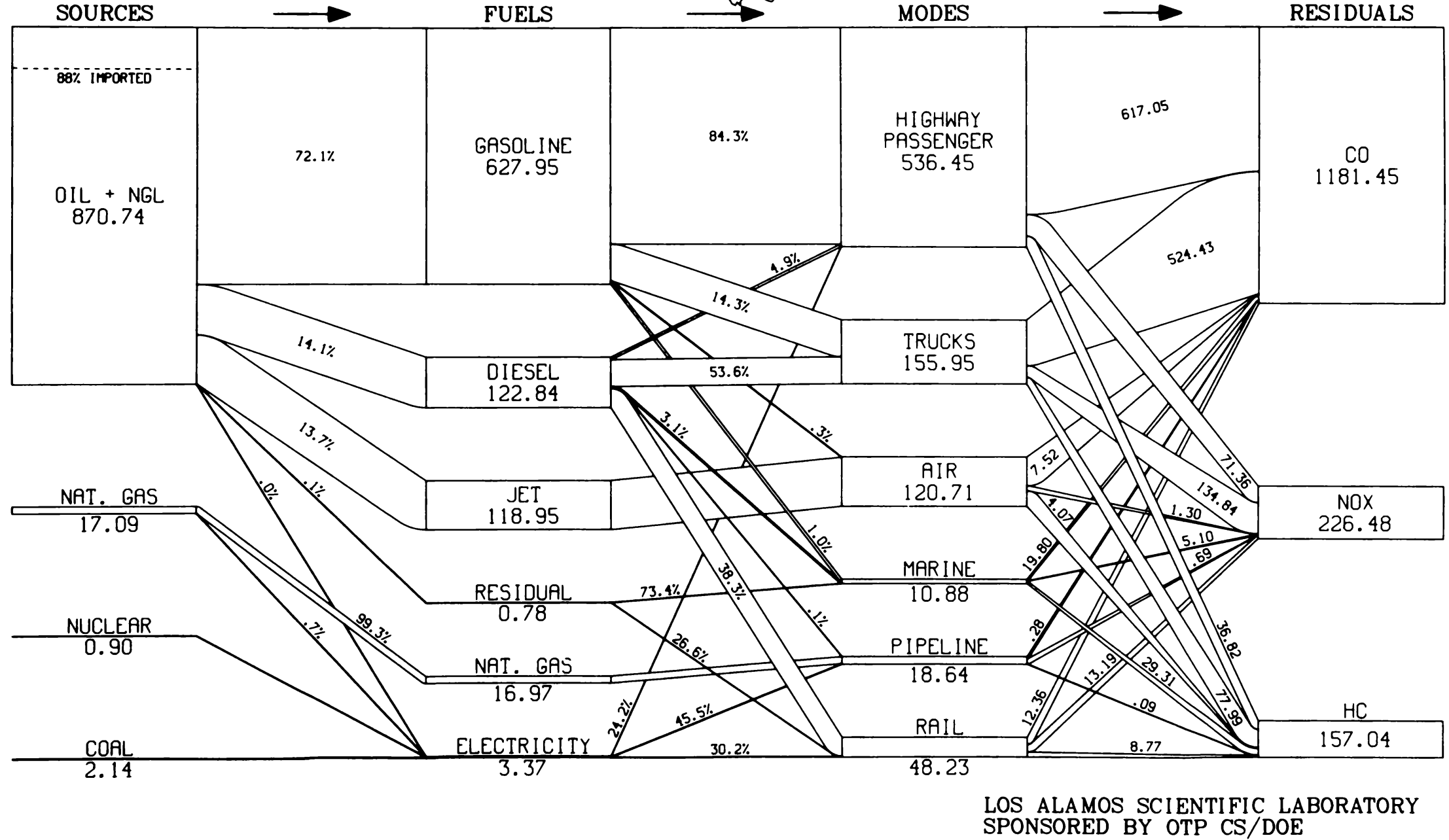

TOTAL ENERGY CONSUMED $=890.86$ POPULATION $=11171000$ $M B T U / C A P I T A=79.75$

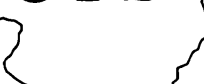

\section{6}

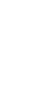




\section{TRANSPORTATION ENERGY FLOWS INDIANA 1976}

UNITS

ENERGY = TRILLIONS OF BTU

POLLUTION = THOUSANDS OF TONS

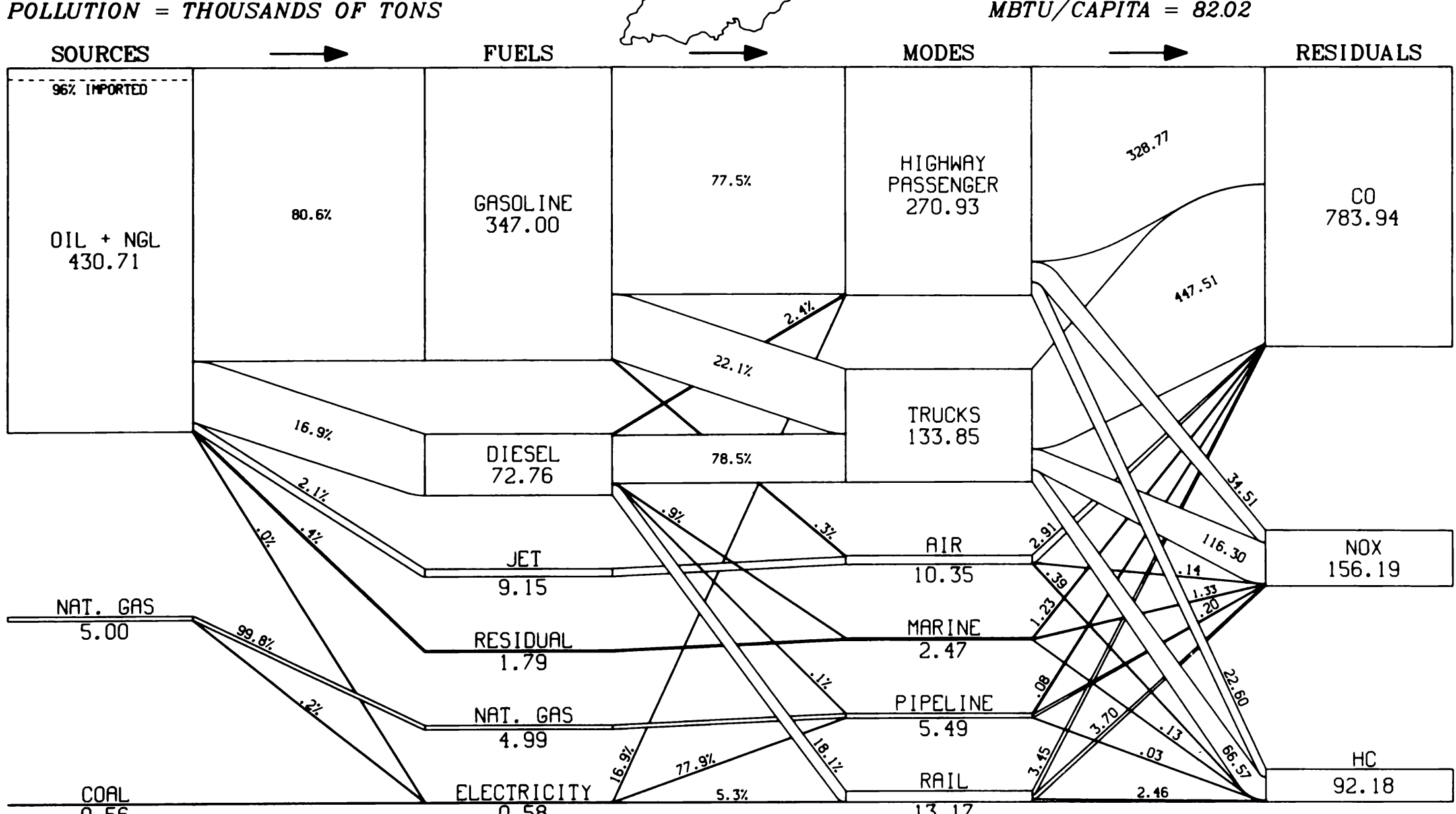

0.58
TOTAL ENERGY CONSUMED $=436.27$

POPULATION $=5319000$

MBTU $/$ CAPITA $=82.02$

\section{(}

13.17

LOS ALAMOS SCIENTIFIC LABORATORY SPONSORED BY OTP CS/DOE 


\section{TRANSPORTATION ENERGY FLOWS IOW A 1976}

\section{UNITS}

ENERGY = TRILLIONS OF BTU

POLLUTION = THOUSANDS OF TONS

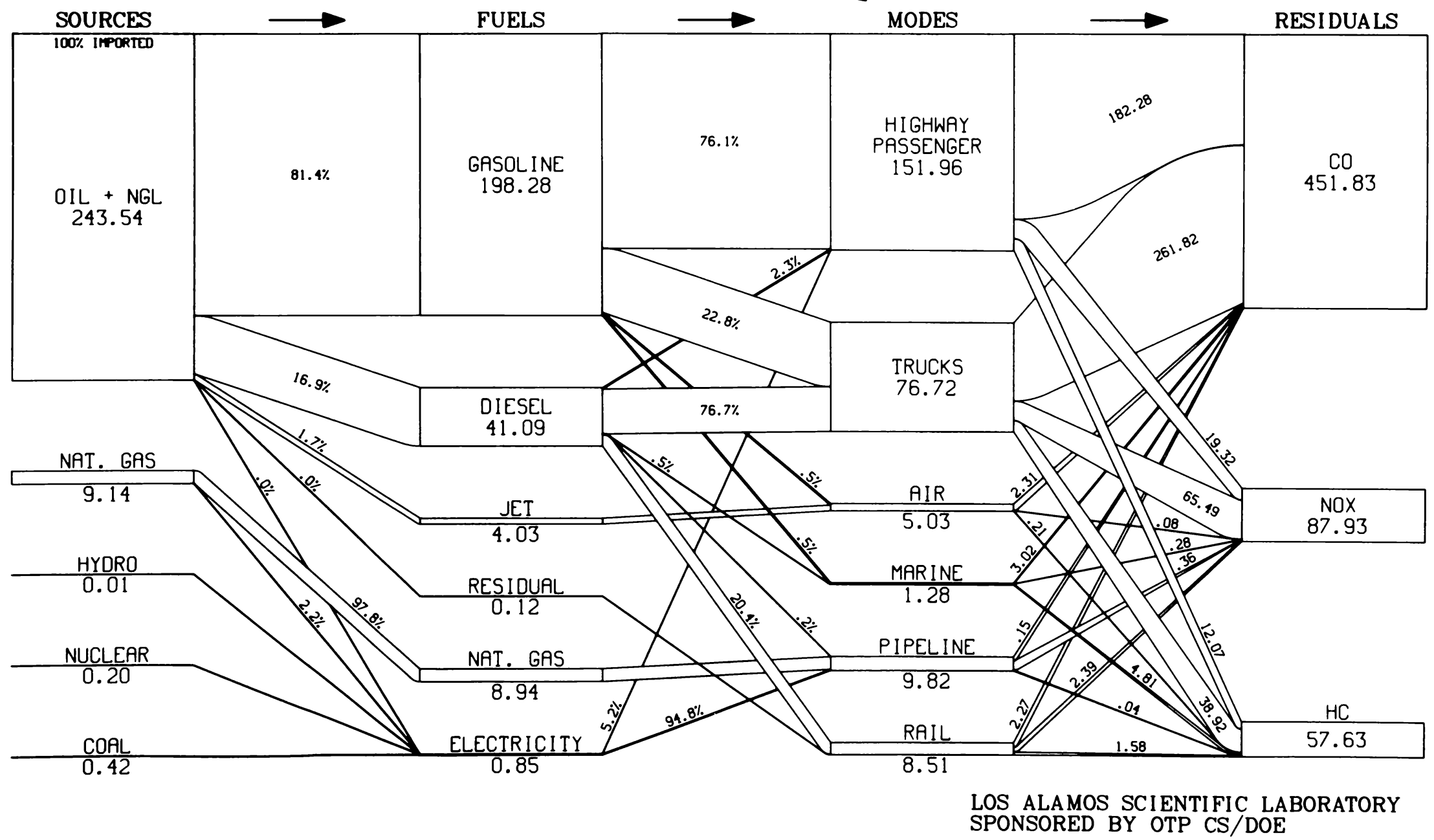

TOTAL ENERGY CONSUMED $=253.31$ POPULATION $=2893000$

MBTU $/$ CAPITA $=87.56$ 


\section{TRANSPORTATION ENERGY FLOWS KANSAS 1976}

\section{UNITS}

ENERCY $=$ TRILLIONS OF BTU

POLLUTION $=$ THOUSANDS OF TONS

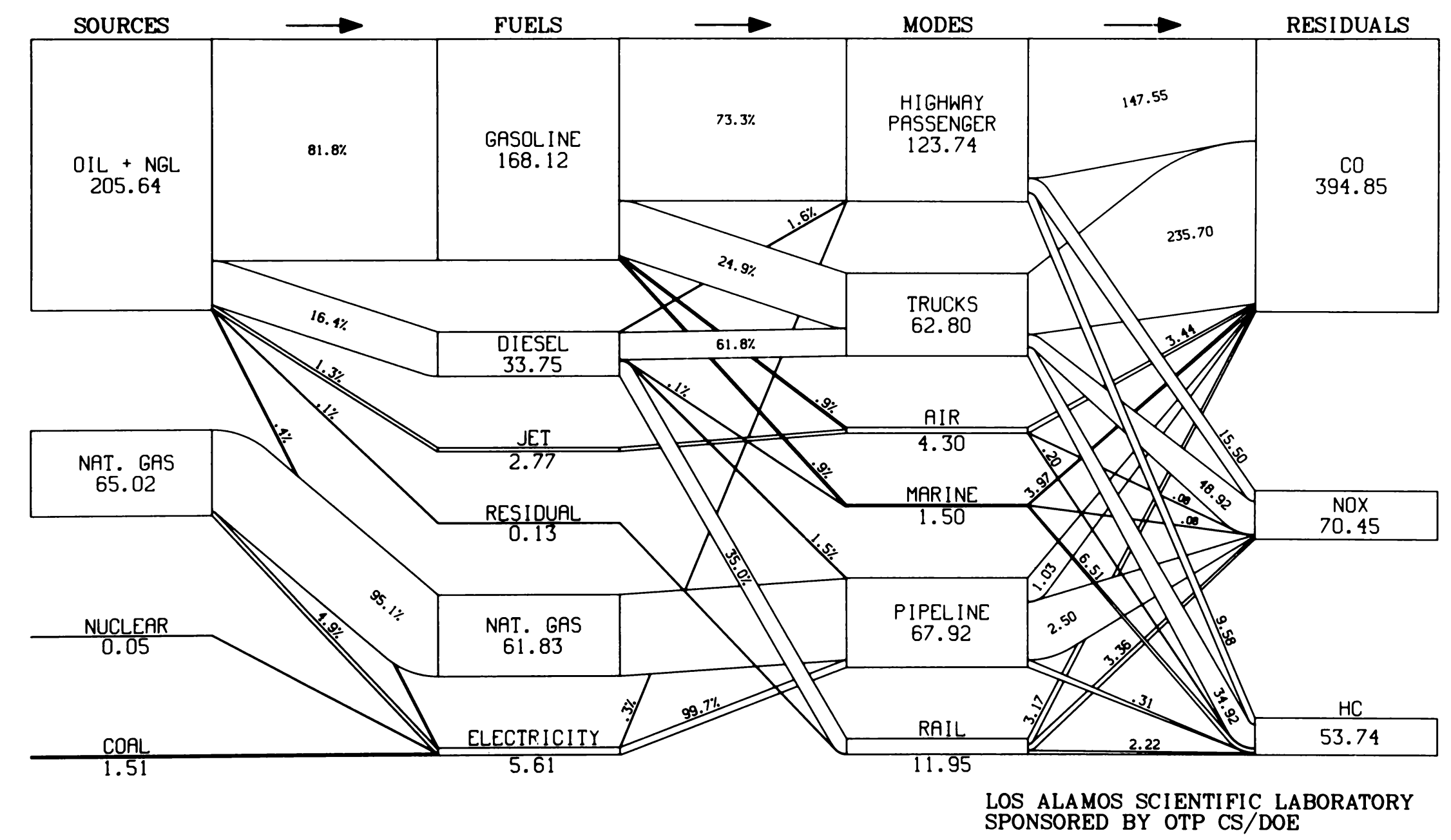

1.51

TOTAL ENERGY CONSUMED $=272.21$ POPULATION $=2283000$ MBTU $/$ CAPITA $=119.23$

LOS ALAMOS SCIENTIFIC LABORATORY 


\section{TRANSPORTATION ENERGY FLOWS KENTUCKY 1976}

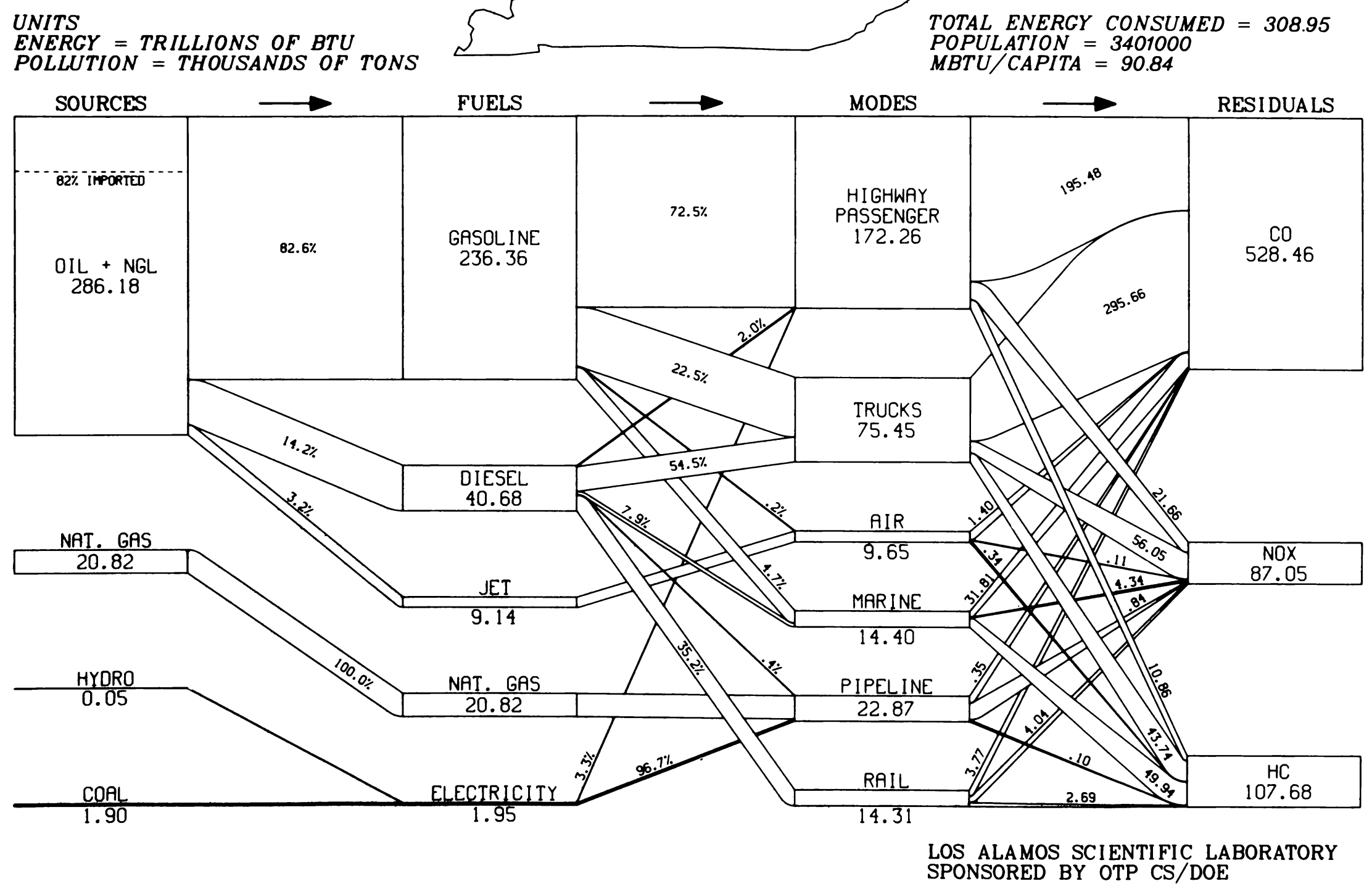




\section{TRANSPORTATION ENERGY FLOWS LOU/ISI ANA 1976}

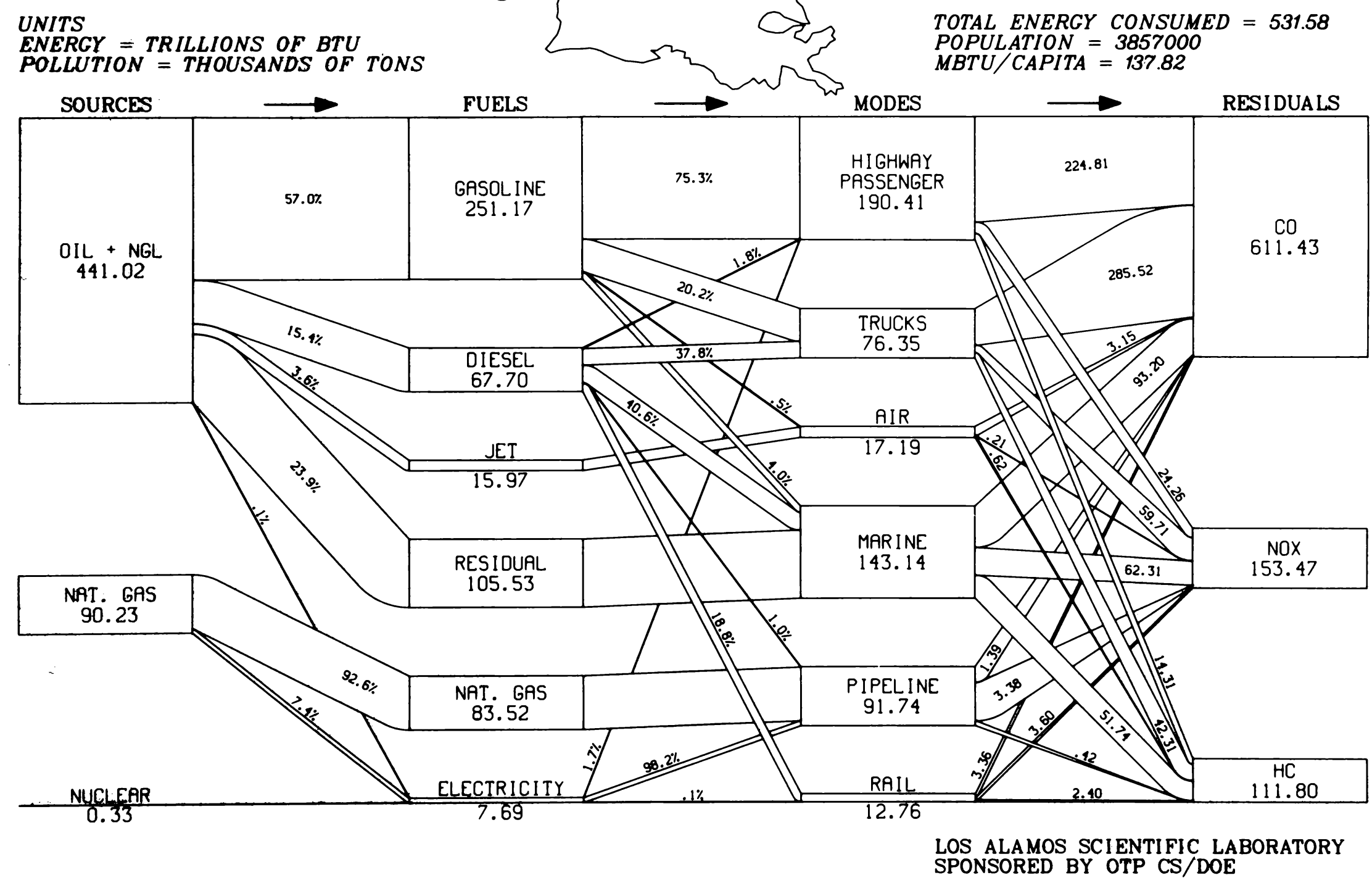




\section{TRANSPORTATION ENERGY FLOWS MAINE 1976}

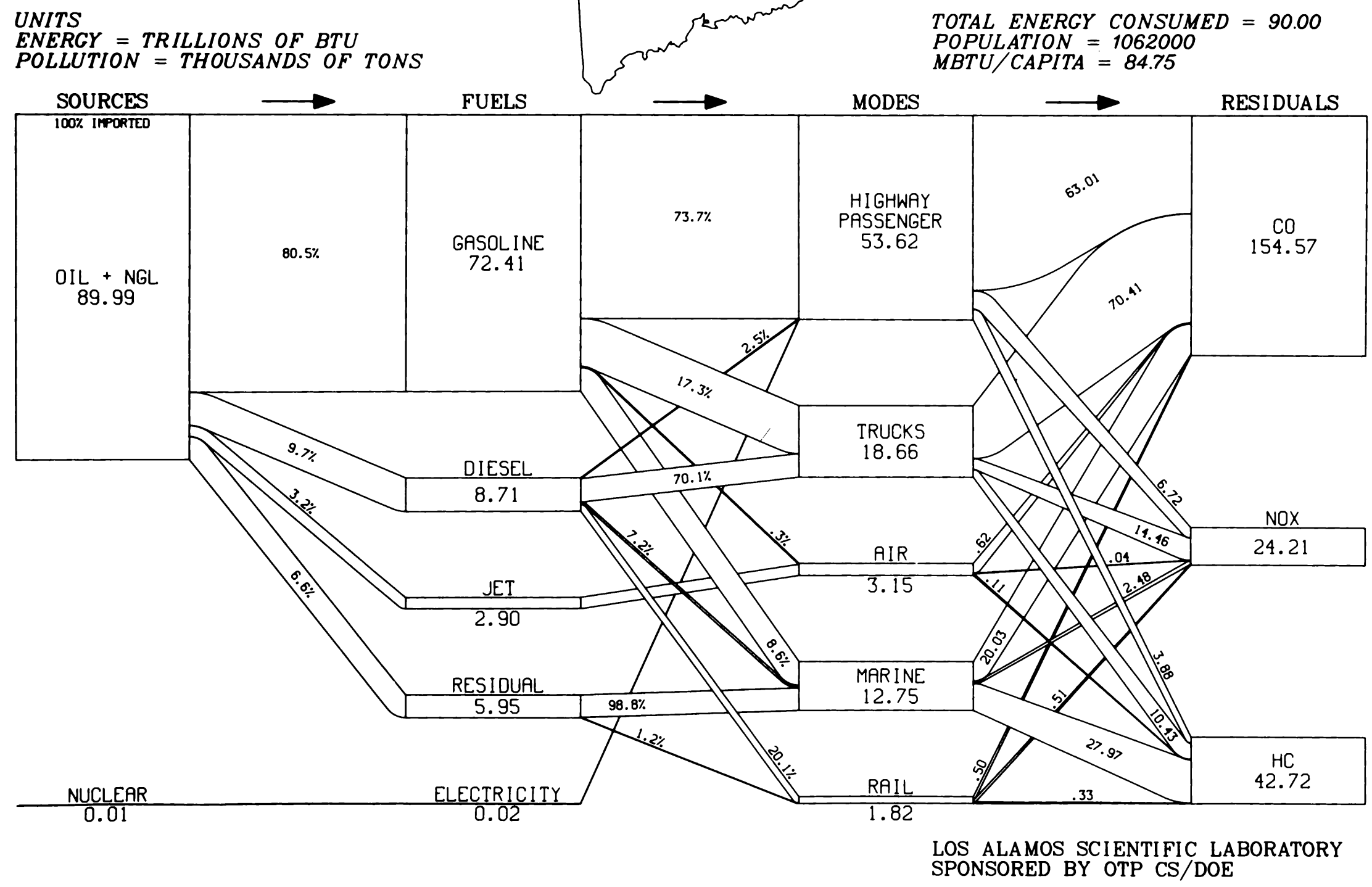




\section{TRANSPORTATION ENERGY FLOWS MARYLAND 1976}

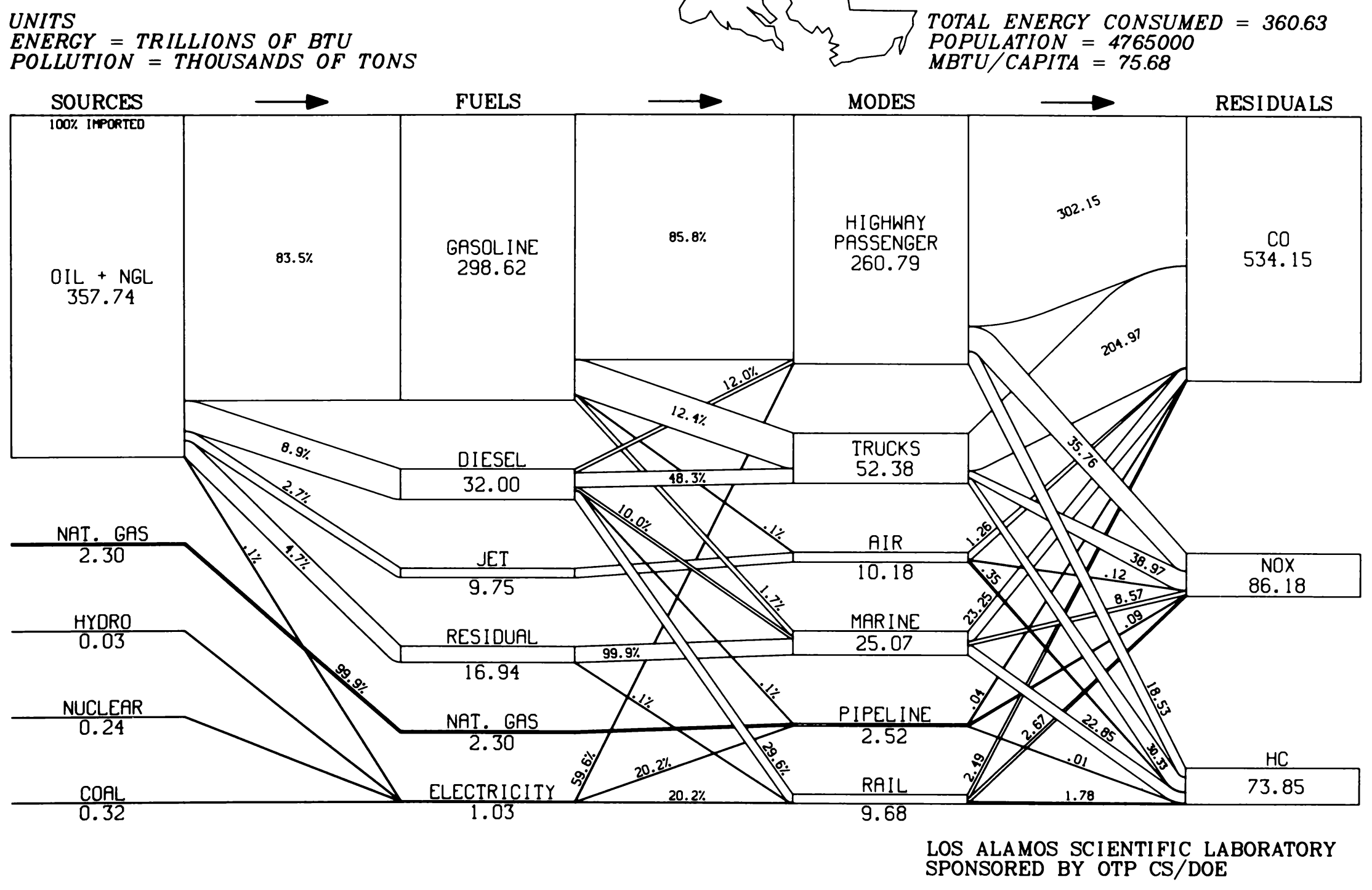




\section{TRANSPORTATION ENERGY FLOWS MASSACHUSETTS 1976}

UNITS

ENERCY = TRILLIONS OF BTU

POLLUTION = THOUSANDS OF TONS

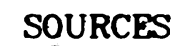

SOURCES

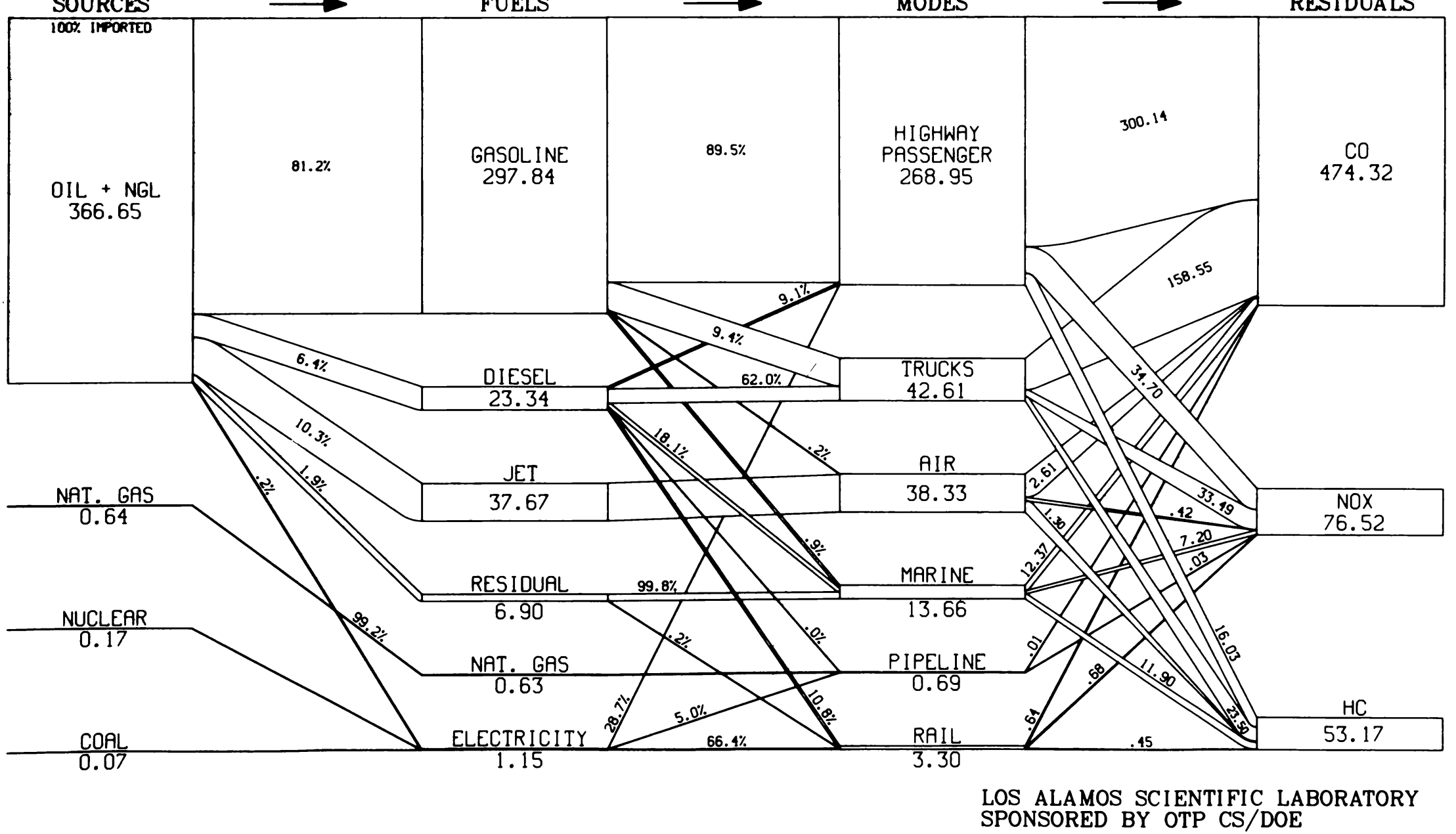

TOTAL ENERGY CONSUMED $=367.53$ POPULATION $=5757000$

MBTU $/ C A P I T A=63.84$

$\Omega$
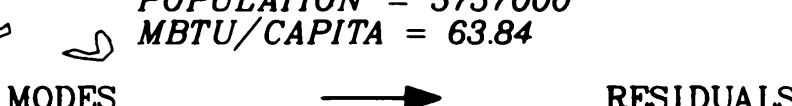
SPONSORED BY OTP CS/DOE 


\section{TRANSPORTATION ENERGY FLOWS MICHIGANN 1976}

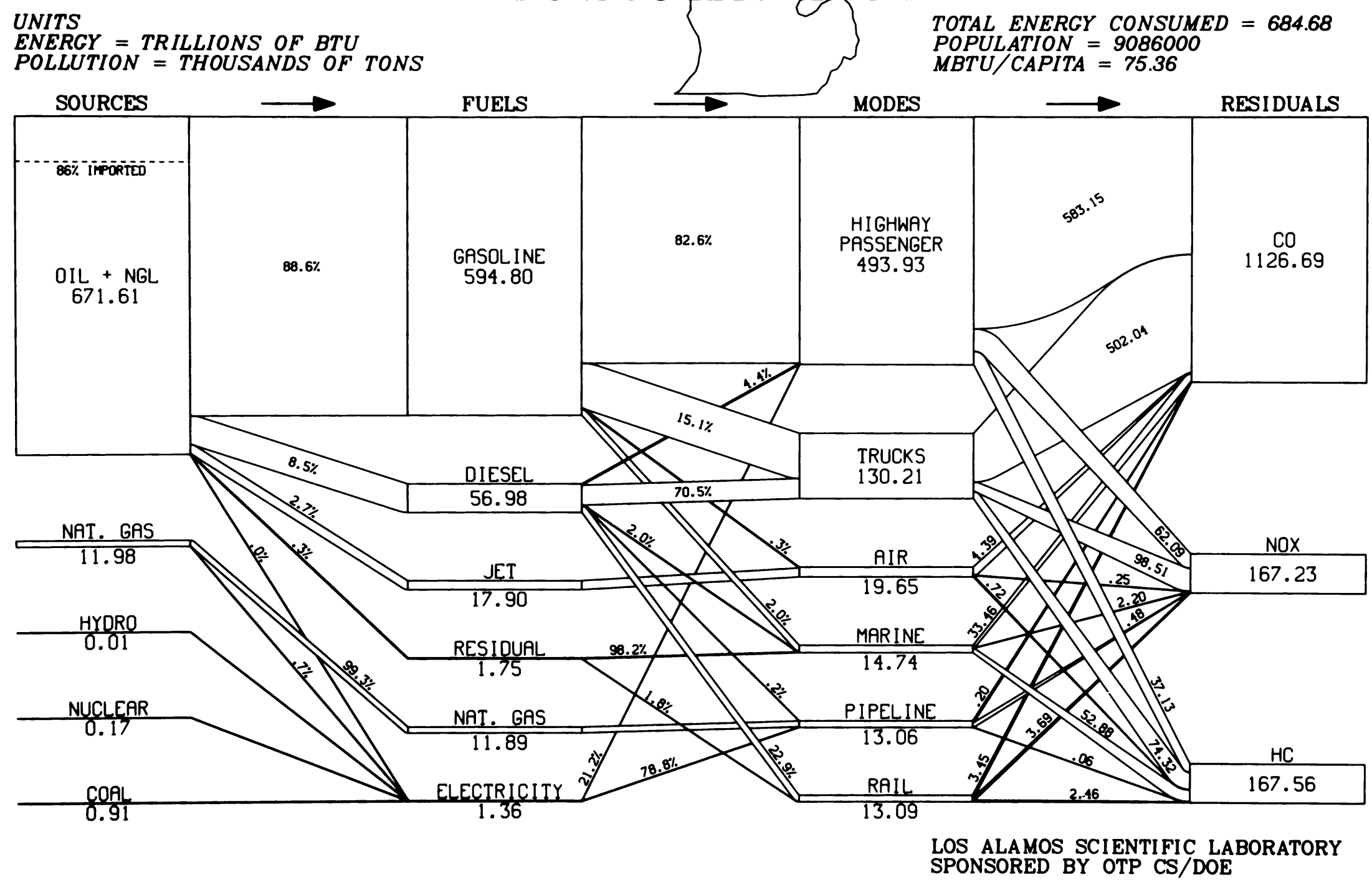




\section{TRANSPORTATION ENERGY FLOWS MINNESOTA 1976}

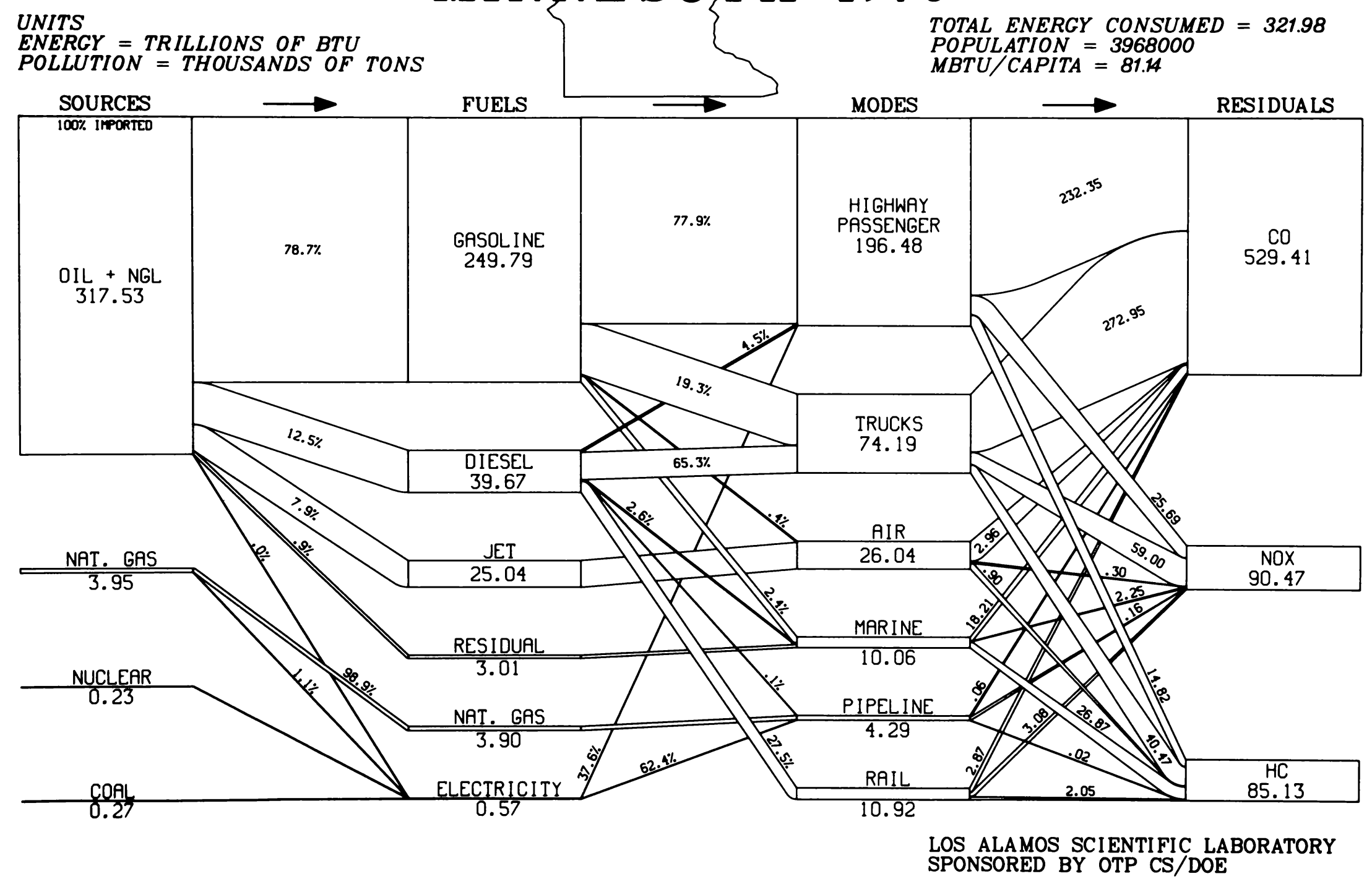




\section{TRANSPORTATION ENERGY FLOWS MISSISSIPPI 1976}

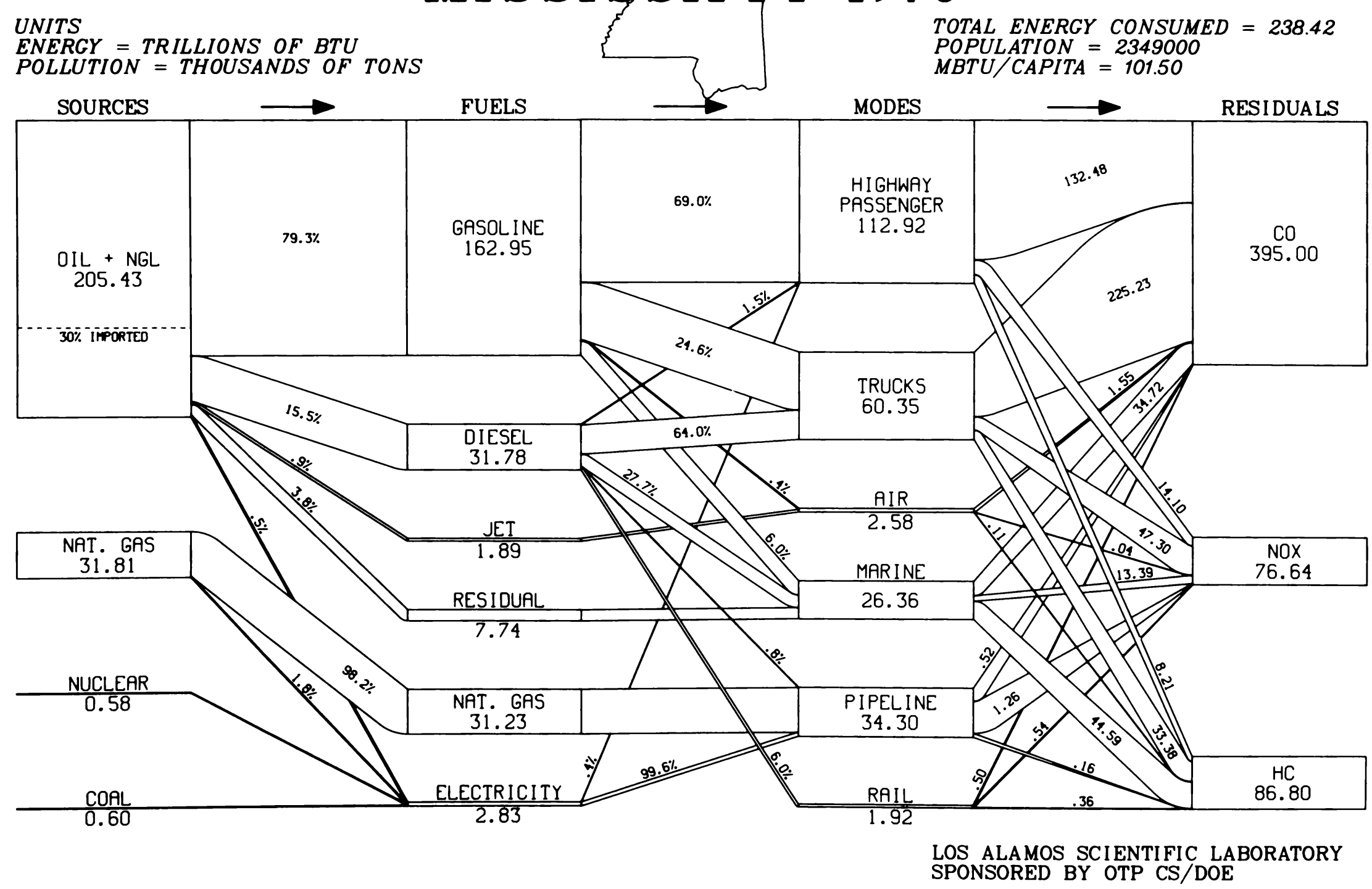




\section{TRANSPORTATION ENERGY FLOWS MISSOURI 1976}

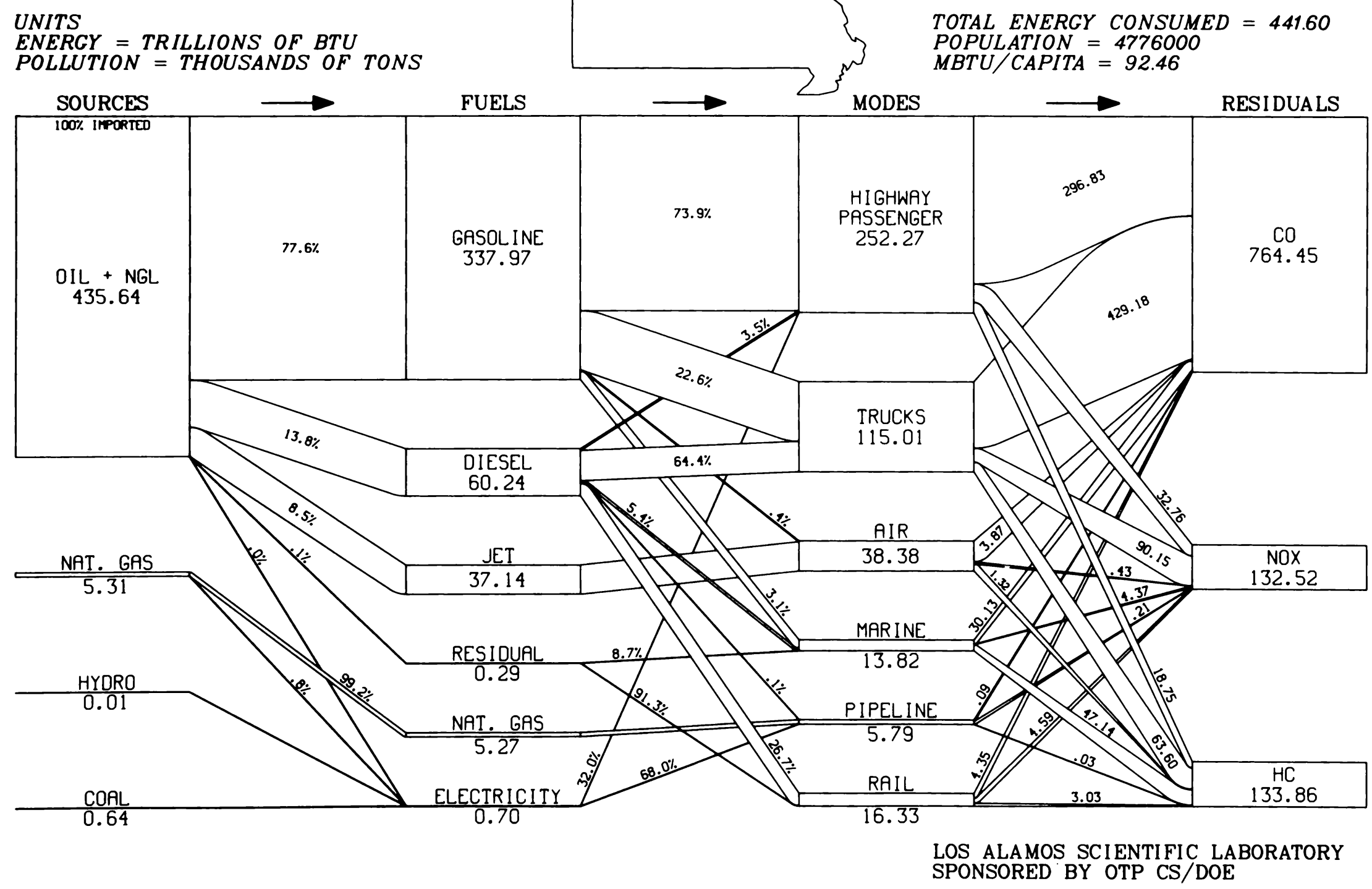




\section{TRANSPORTATION ENERGY FLOWS MONTANA 1976}
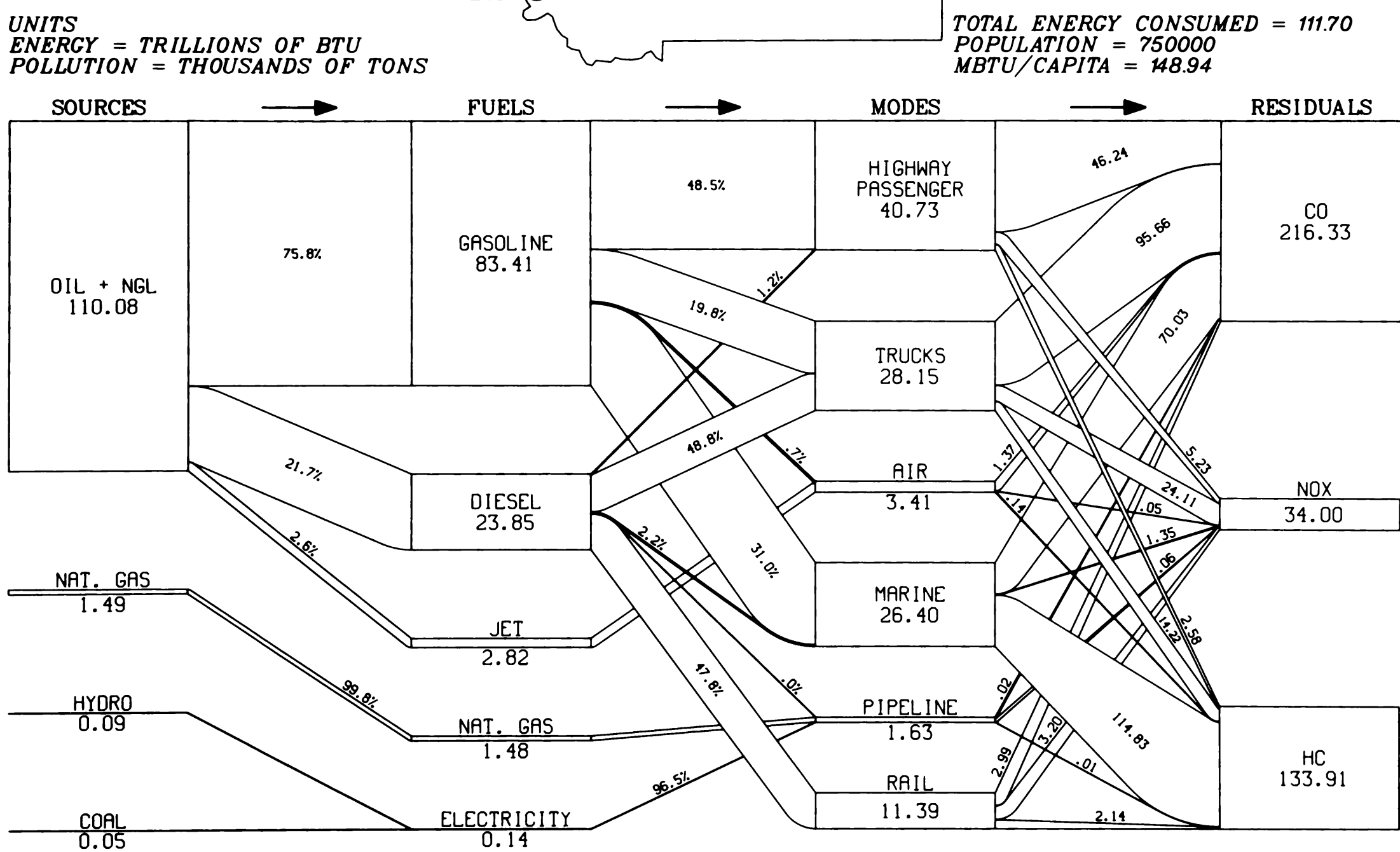

LOS ALAMOS SCIENTIFIC LABORATORY 


\section{TRANSPORTATION ENERGY FLOWS NEBRASKA 1976}

\section{UNITS}

ENERCY = TRILLIONS OF BTU

POLLUTION = THOUSANDS OF TONS

SOURCES

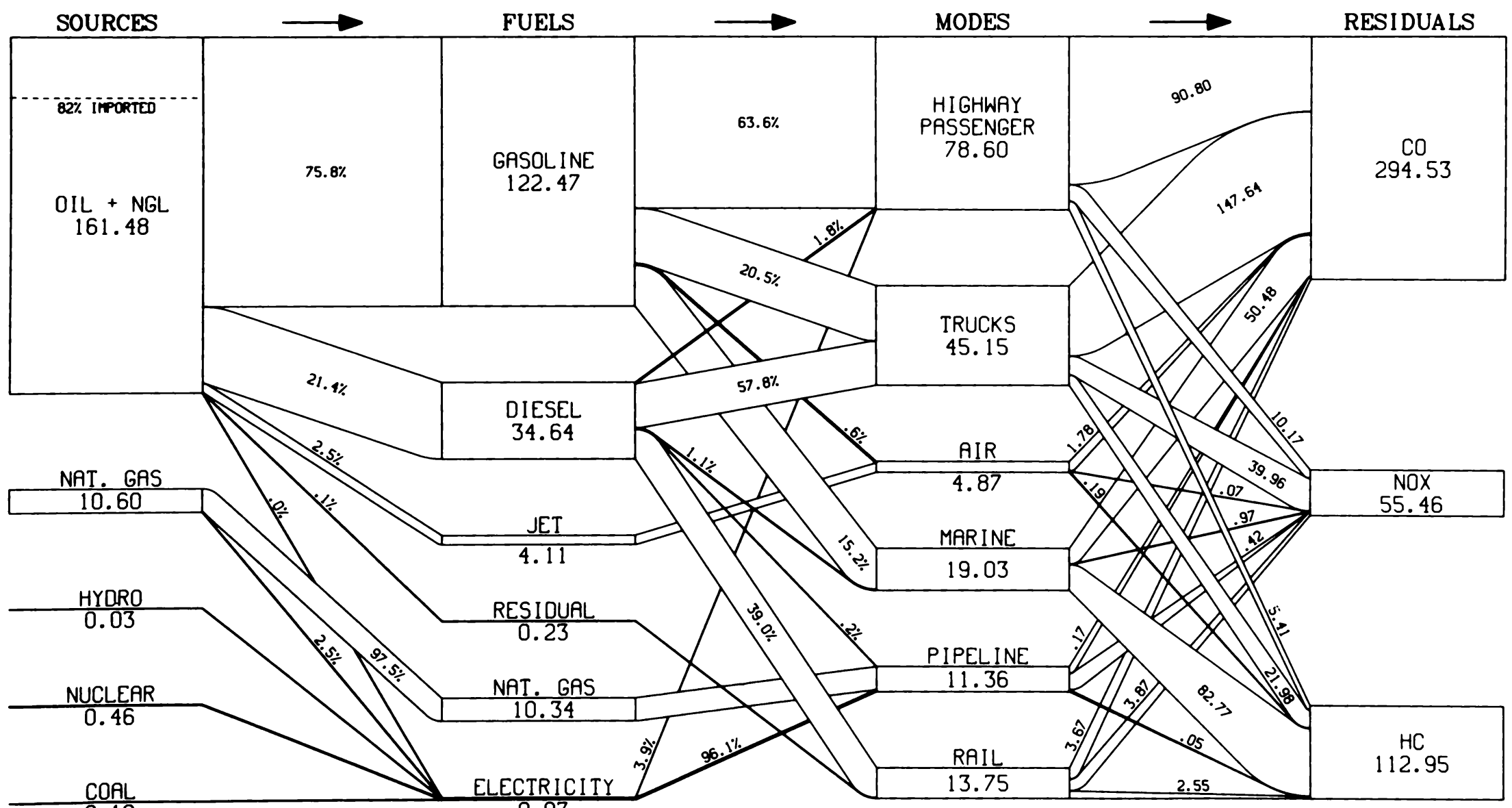

0.19
0.97
TOTAL ENERGY CONSUMED = 172.76 POPULATION $=1543000$ $M B T U / C A P I T A=111.96$

FUELS RESIDUALS LOS ALAMOS SCIENTIFIC LABORATORY SPONSORED BY OTP CS/DOE 


\section{TRANSPORTATION ENERGY FLOWS NEVADA 1976}

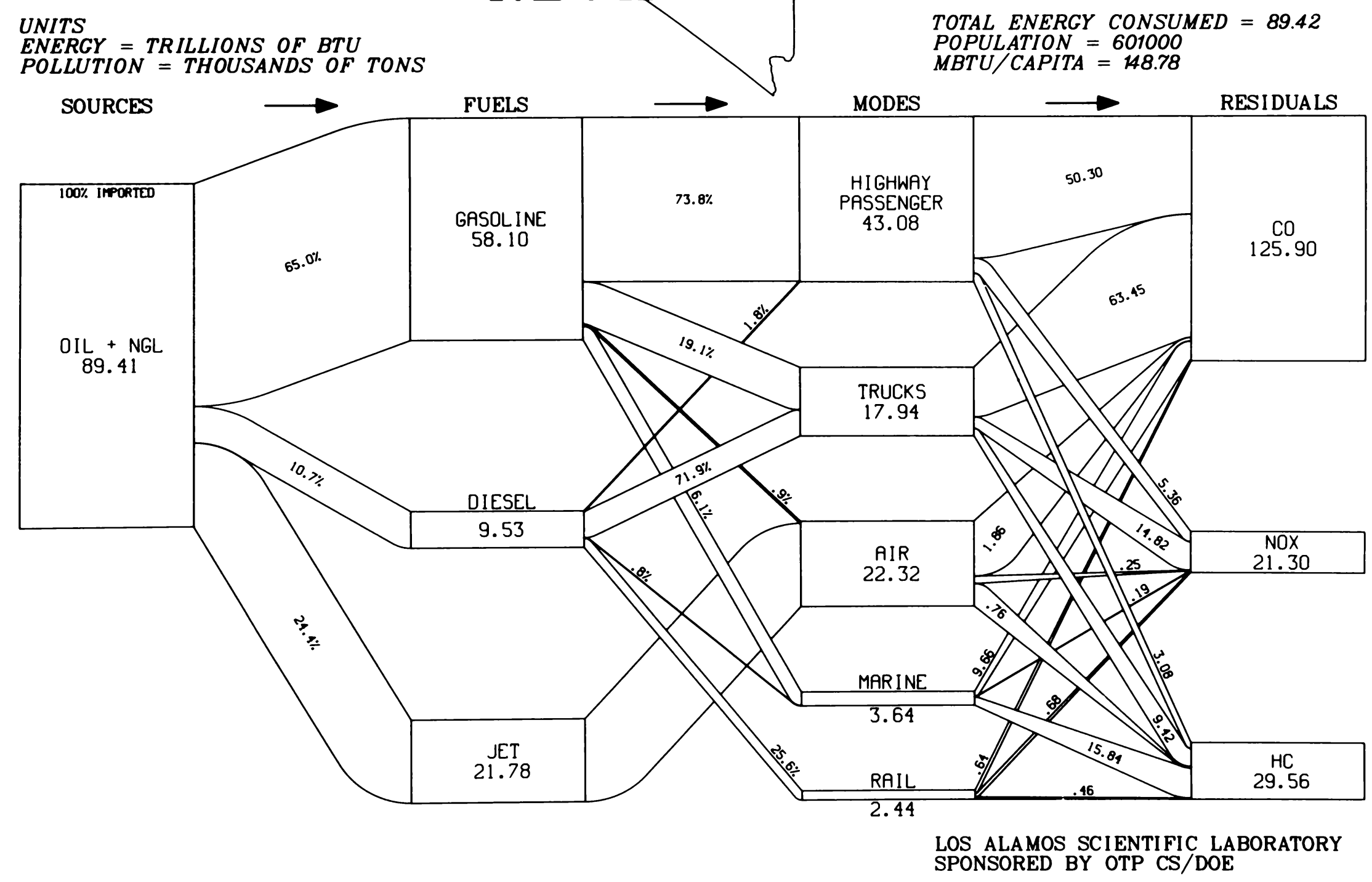




\section{TRANSPORTATION ENERGY FLOWS NEW HAMPSHIRE 1976}

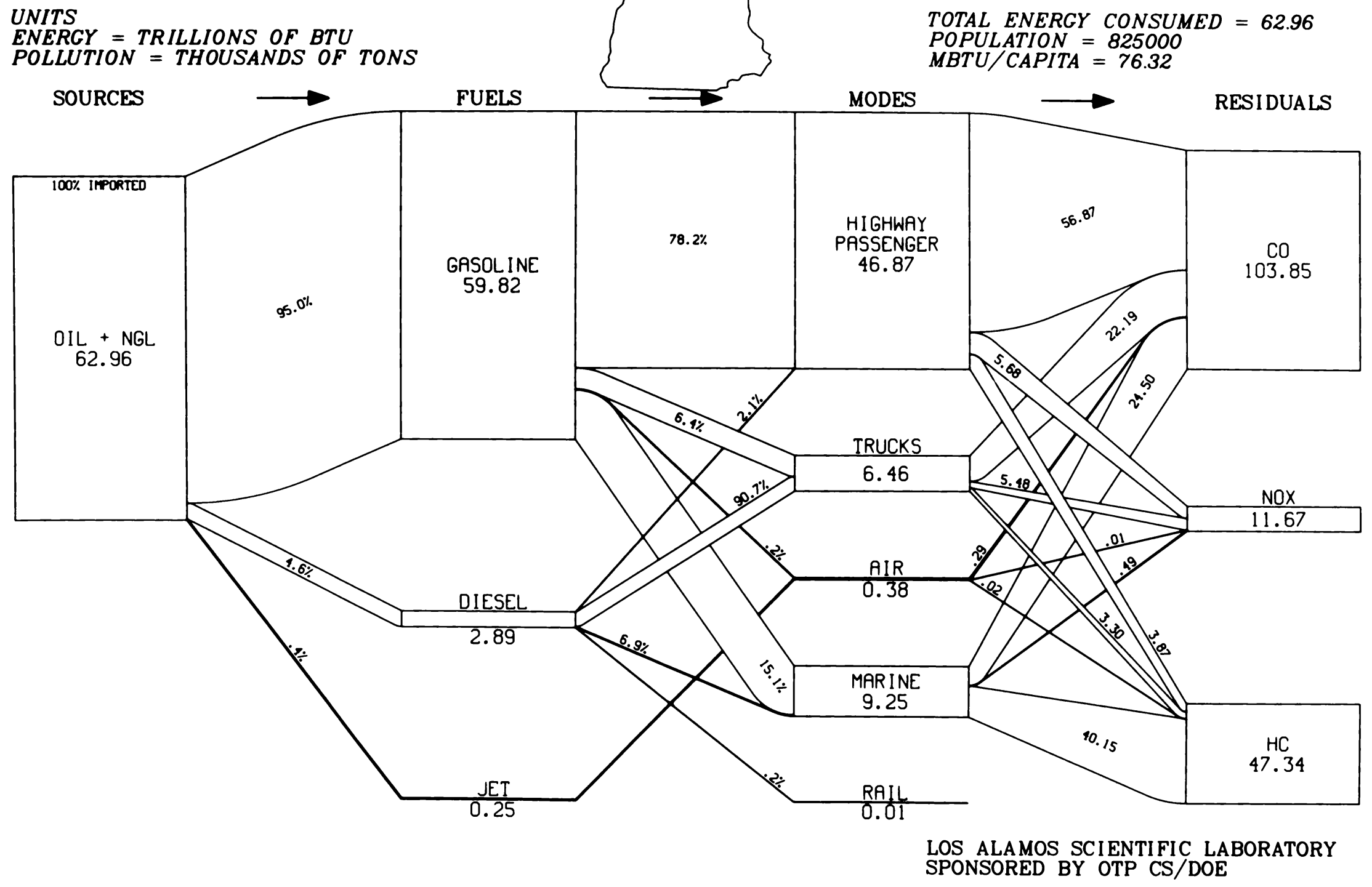




\section{TRANSPORTATION ENERGY FLOWS NEW JERSEY 1976}

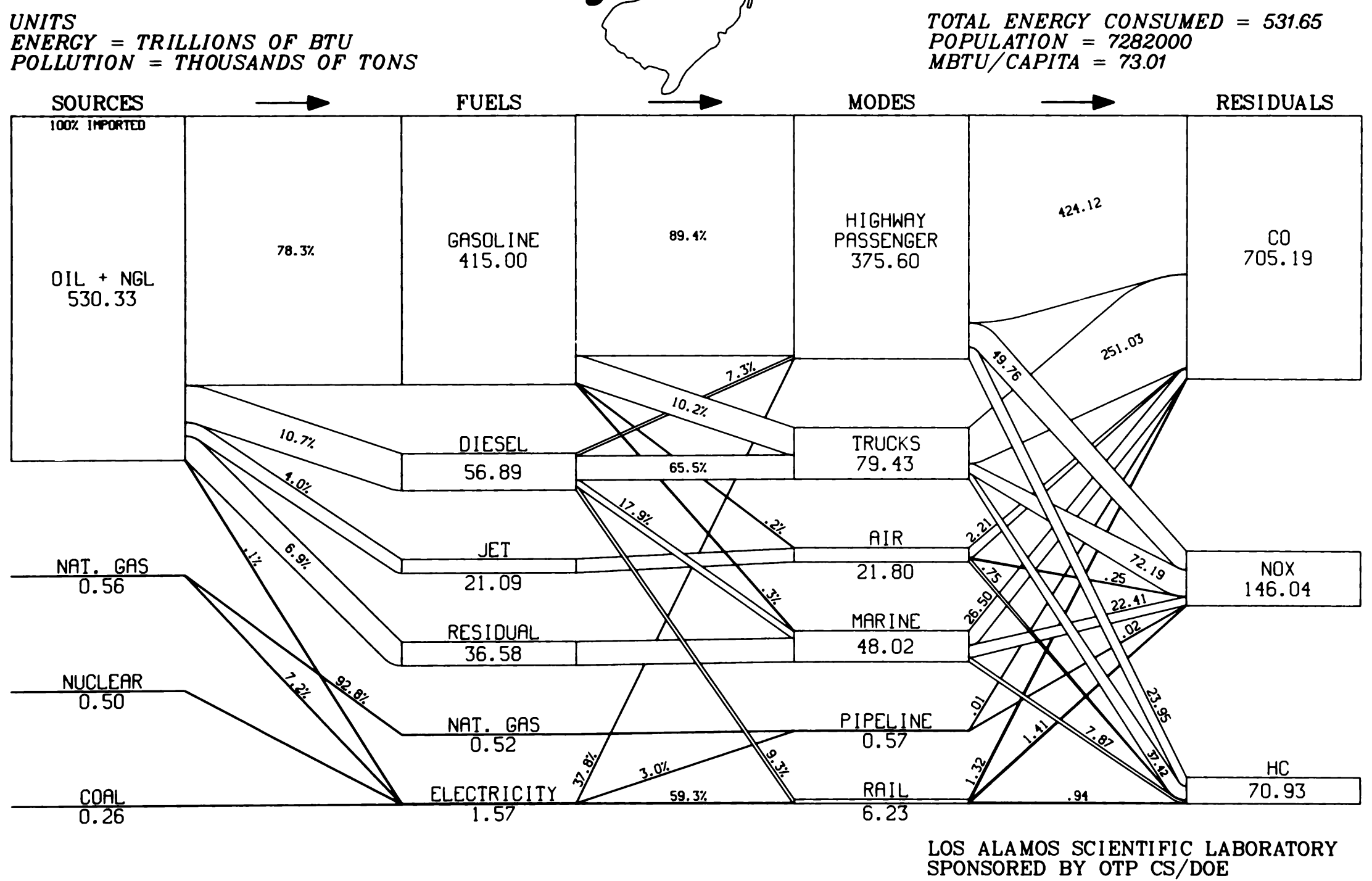




\section{TRANSPORTATION ENERGY FLOWS NEW MEXICO 1976}

\section{UNITS}

ENERCY = TRILLIONS OF BTU

POLLUTION = THOUSANDS OF TONS

SOURCES

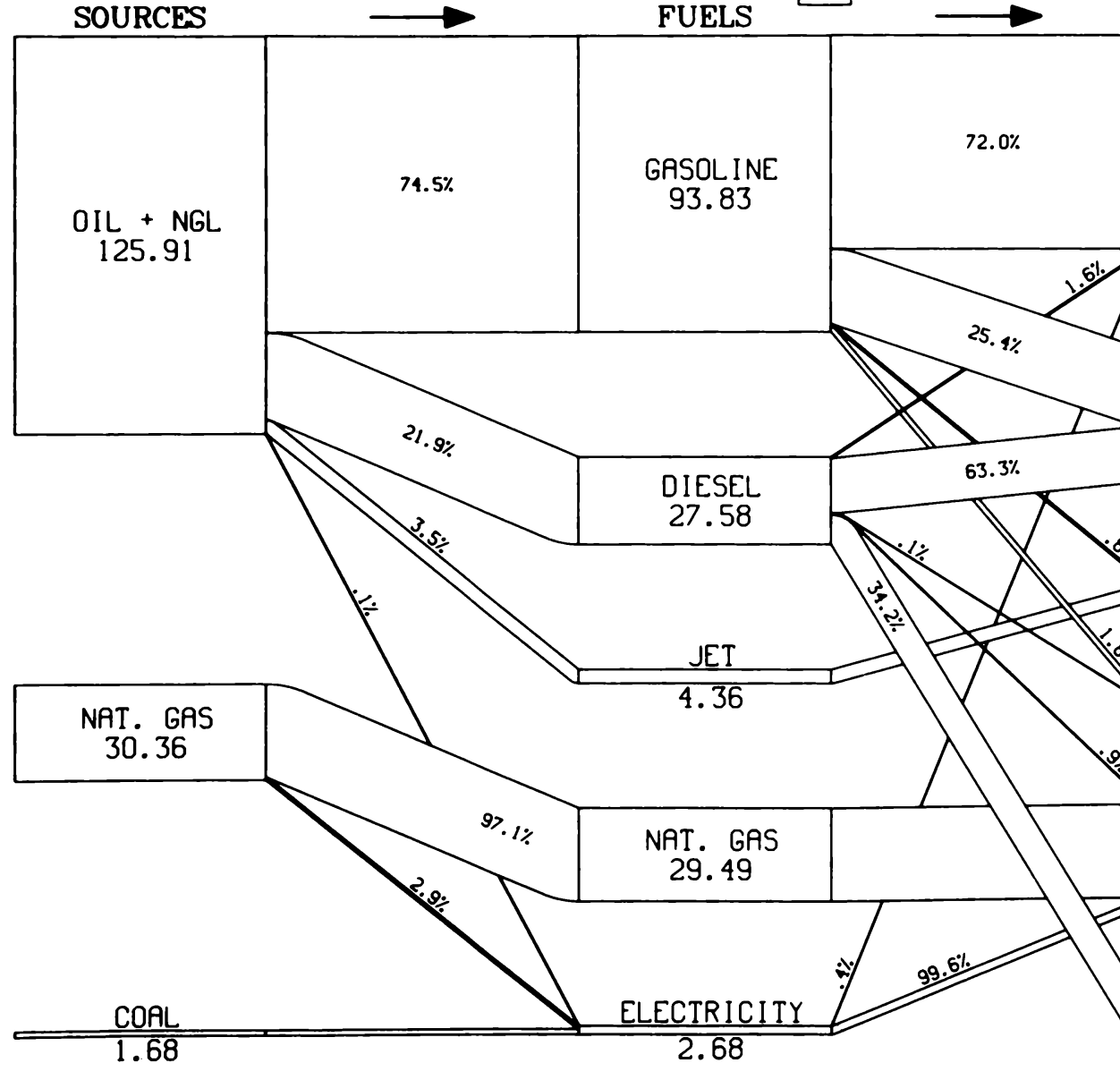

TOTAL ENERGY CONSUMED $=157.94$ POPULATION $=1156000$ $M B T U / C A P I T A=136.63$

MODES $\longrightarrow$ RESIDUALS

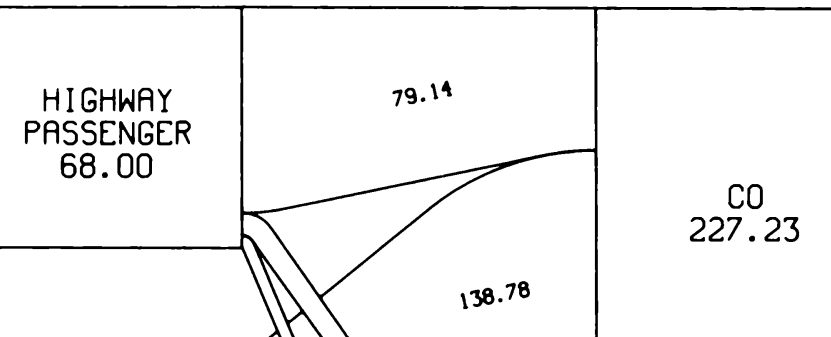

TRUCKS 41.29

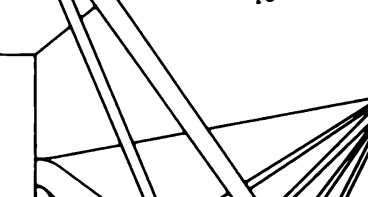

AIR
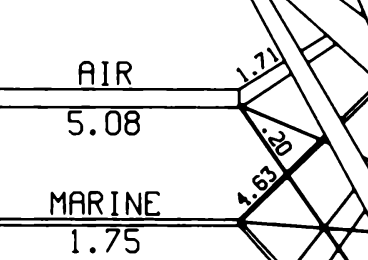

1.75

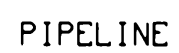
32.39

RAIL
9.43

ALAMOS SCIENTIFIC LABORATORY SPONSORED BY OTP CS/DOE 


\section{TRANSPORTATION ENERGY FLOWS NEW YORK 1976}

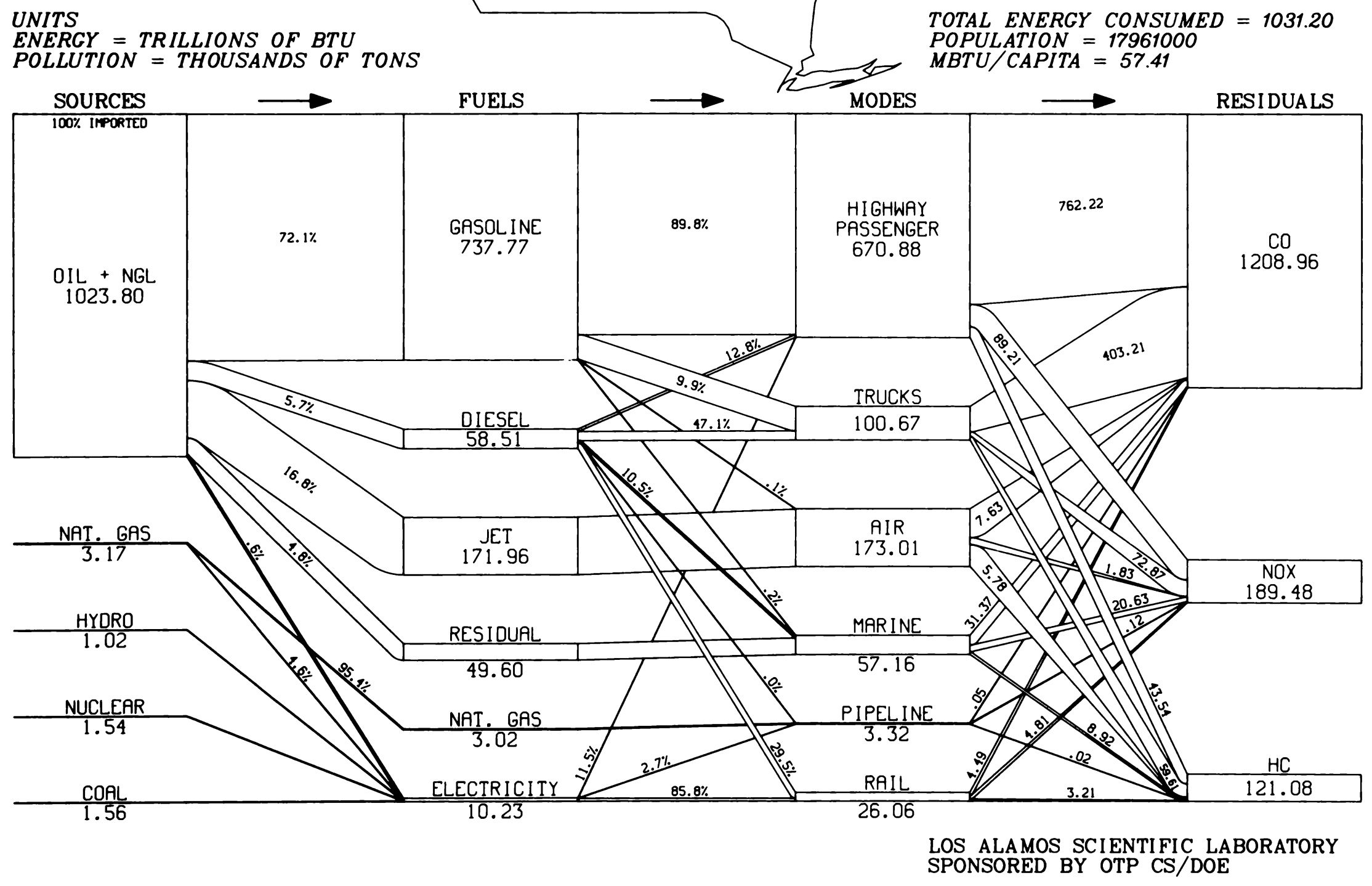




\section{TRANSPORTATION ENERGY FLOWS NORTH CAROLINA 1976}

UNITS

ENERGY = TRILLIONS OF BTU

POLLUTION = THOUSANDS OF TONS

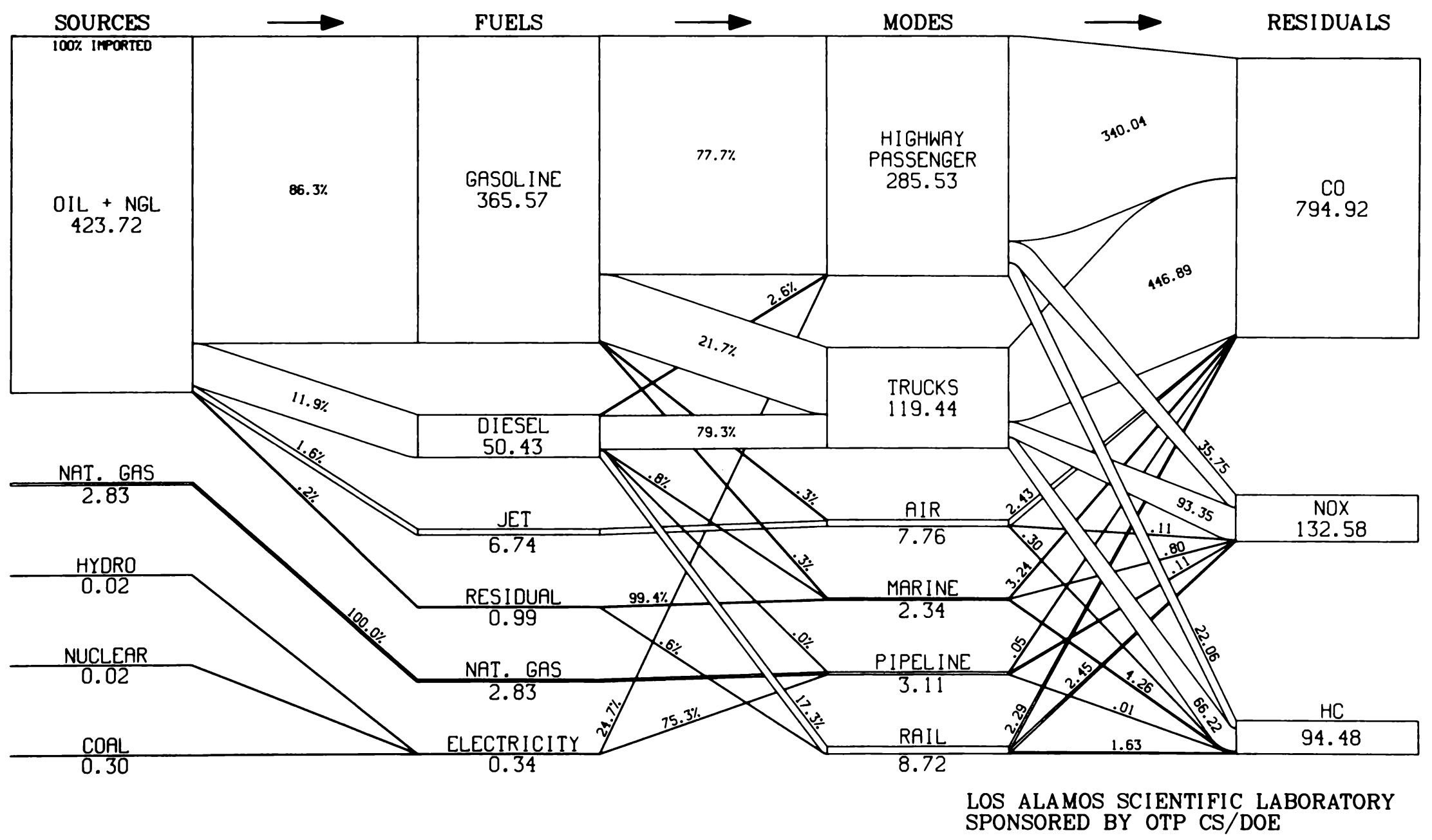




\section{TRANSPORTATION ENERGY FLOWS NORTH DAKOTA 1976}

UNITS
ENERGY $=$ TRILLIONS OF BTU
POLLUTION $=$ THOUSANDS OF TONS

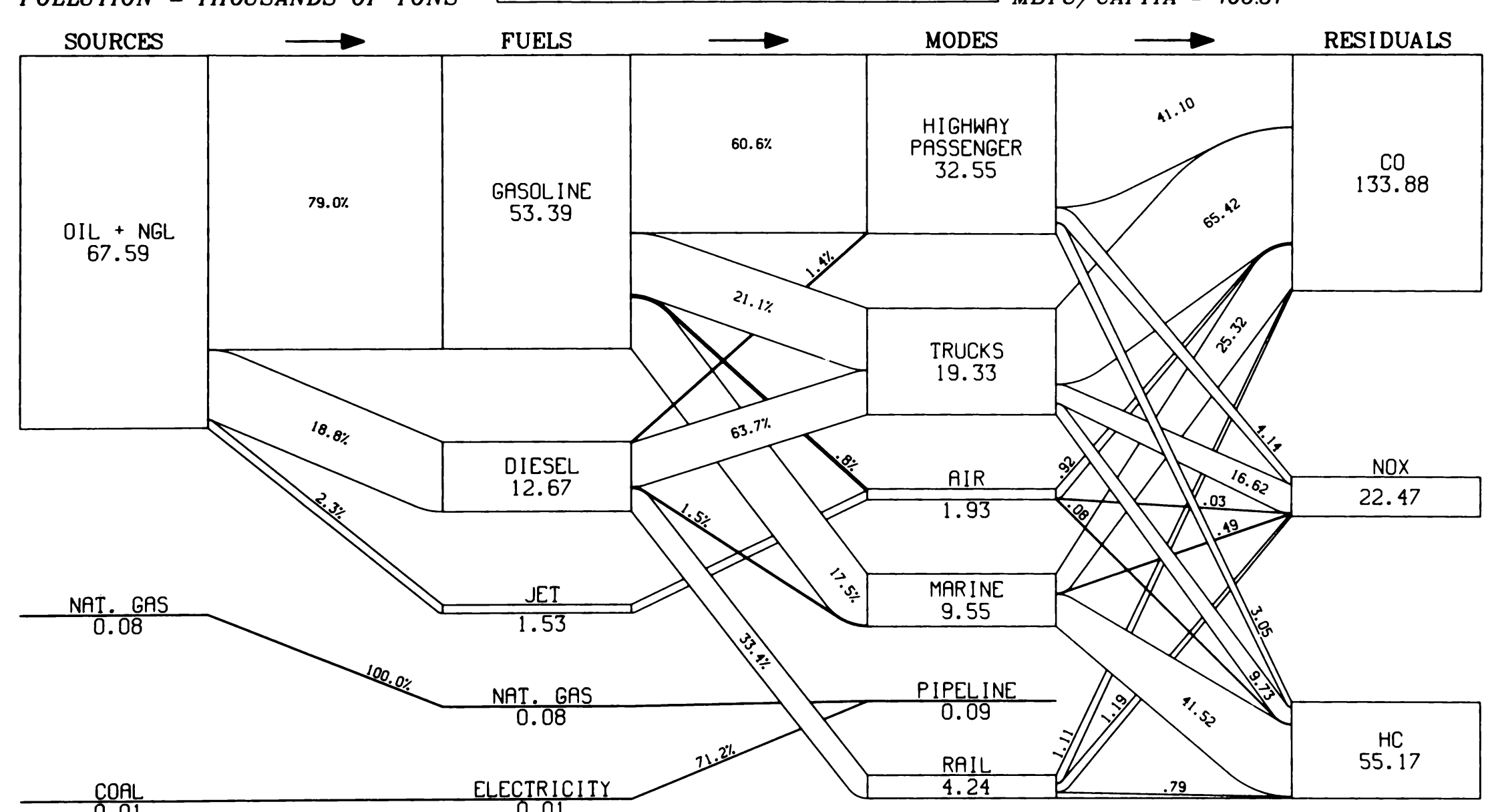




\section{TRANSPORTATION ENERGY FLOWS OHIO 1976}

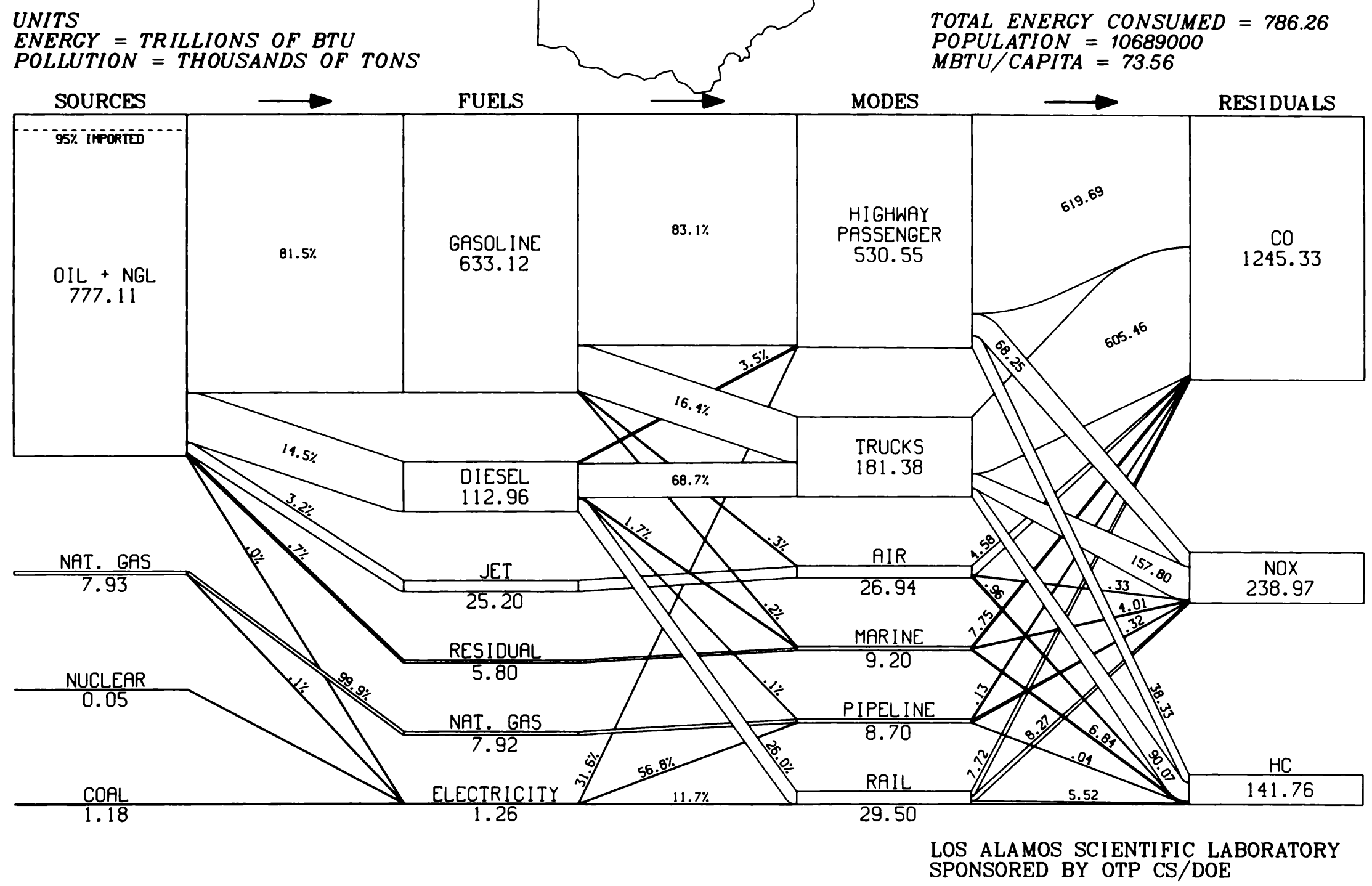




\section{TRANSPORTATION ENERGY FLOWS OKLAHOMA 1976}

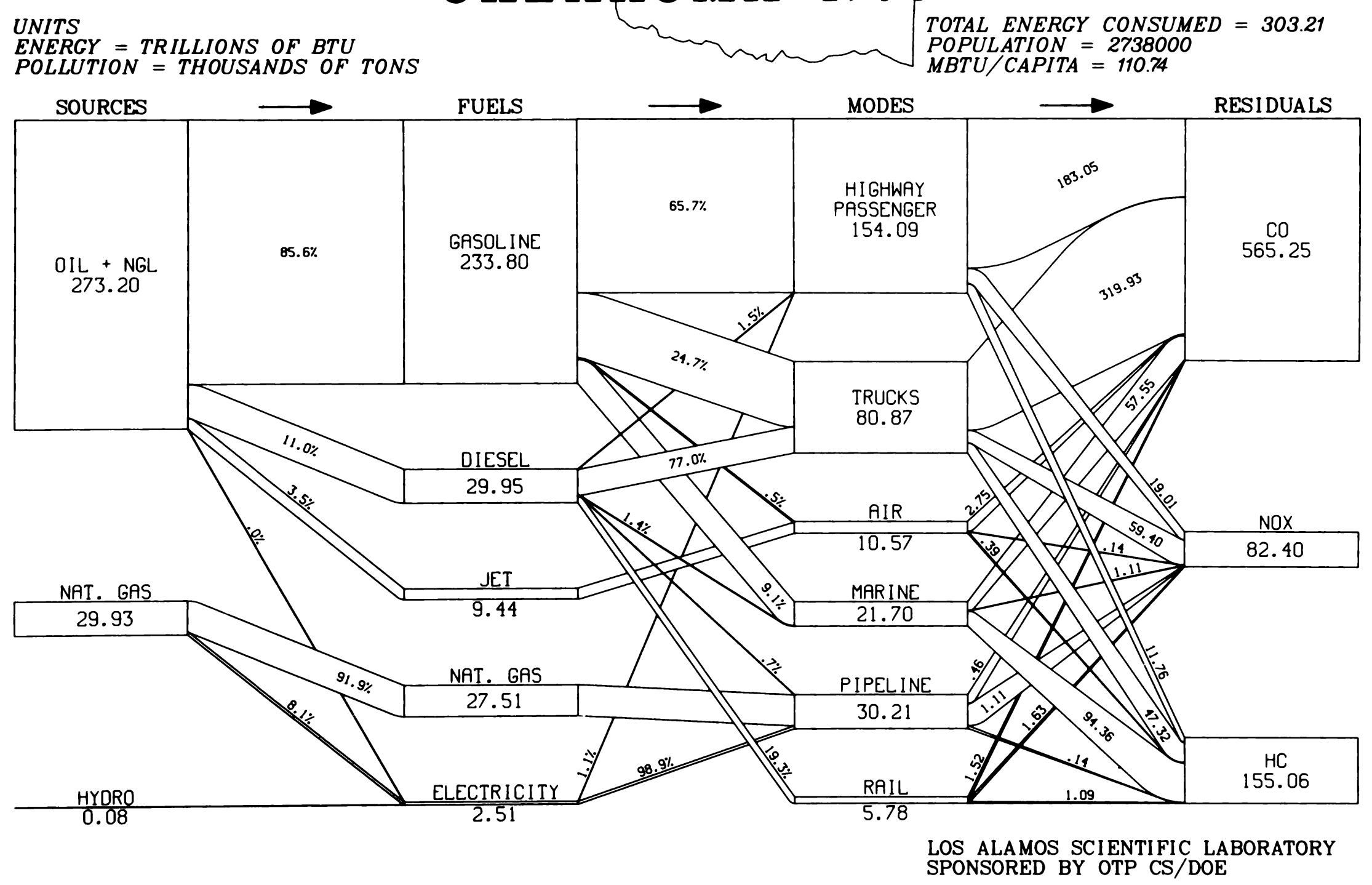




\section{TRANSPORTATION ENERGY FLOWS} OREGON 1976

\section{UNITS}

ENERCY = TRILLIONS OF BTU POLLUTION = THOUSANDS OF TONS
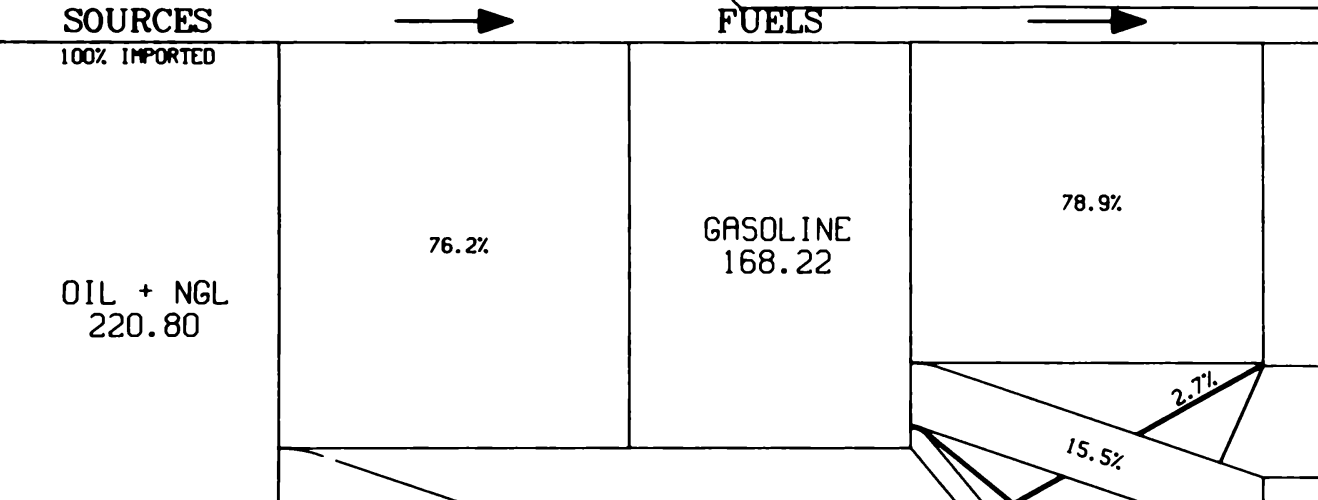

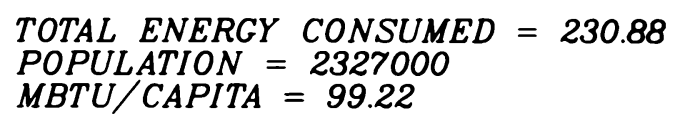

TOTAL ENERGY CONSUMED $=230.88$ POPULATION $=2327000$ $M B T U / C A P I T A=99.22$

MODES
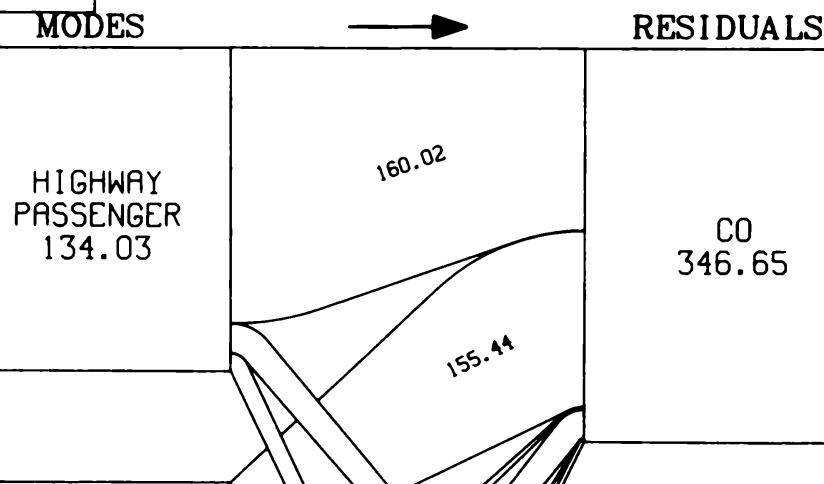

TRUCKS

49.13

$56.0 \%$
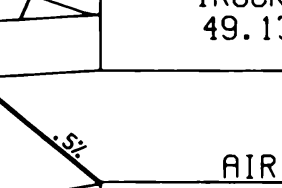

AIR

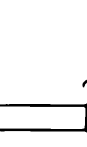

2.)

MARINE

13.24

RESIDUAL

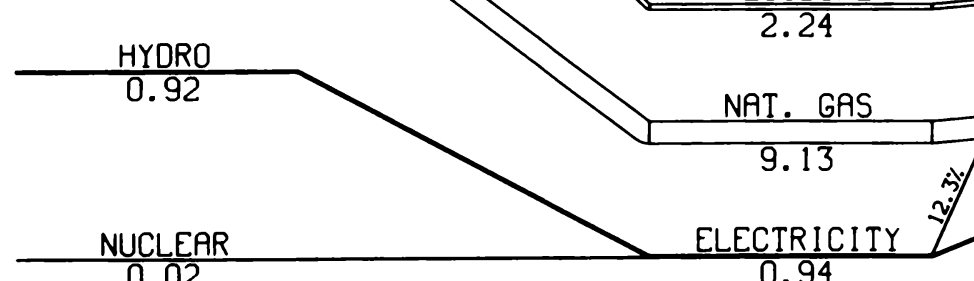




\section{TRANSPORTATION ENERGY FLOWS PENNSYLVANIA 1976}

UNITS
ENERGY $=$ TRILLIONS OF BTU ENERGY $=$ TRILLIONS OF BTU
POLLUTION $=$ THOUSANDS OF TONS

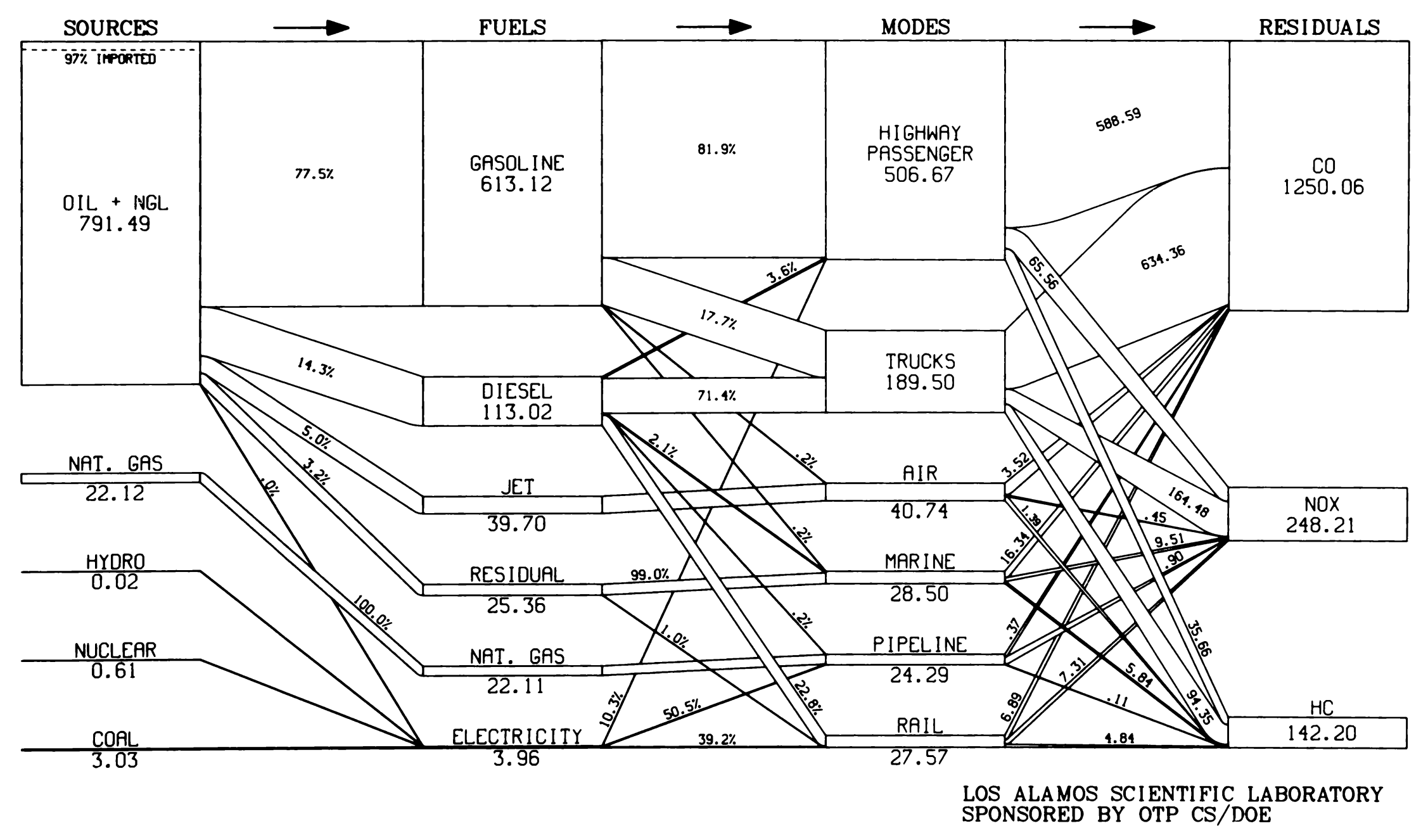
TOTAL ENERGY CONSUMED $=817.27$
POPULATION $=11796000$ $M B T U / C A P I T A=69.28$

SOURCES

FUELS

MODES

RESIDUALS

SPONSORED BY OTP CS/DOE 


\section{TRANSPORTATION ENERGY FLOWS RHODE ISLAND 1976}

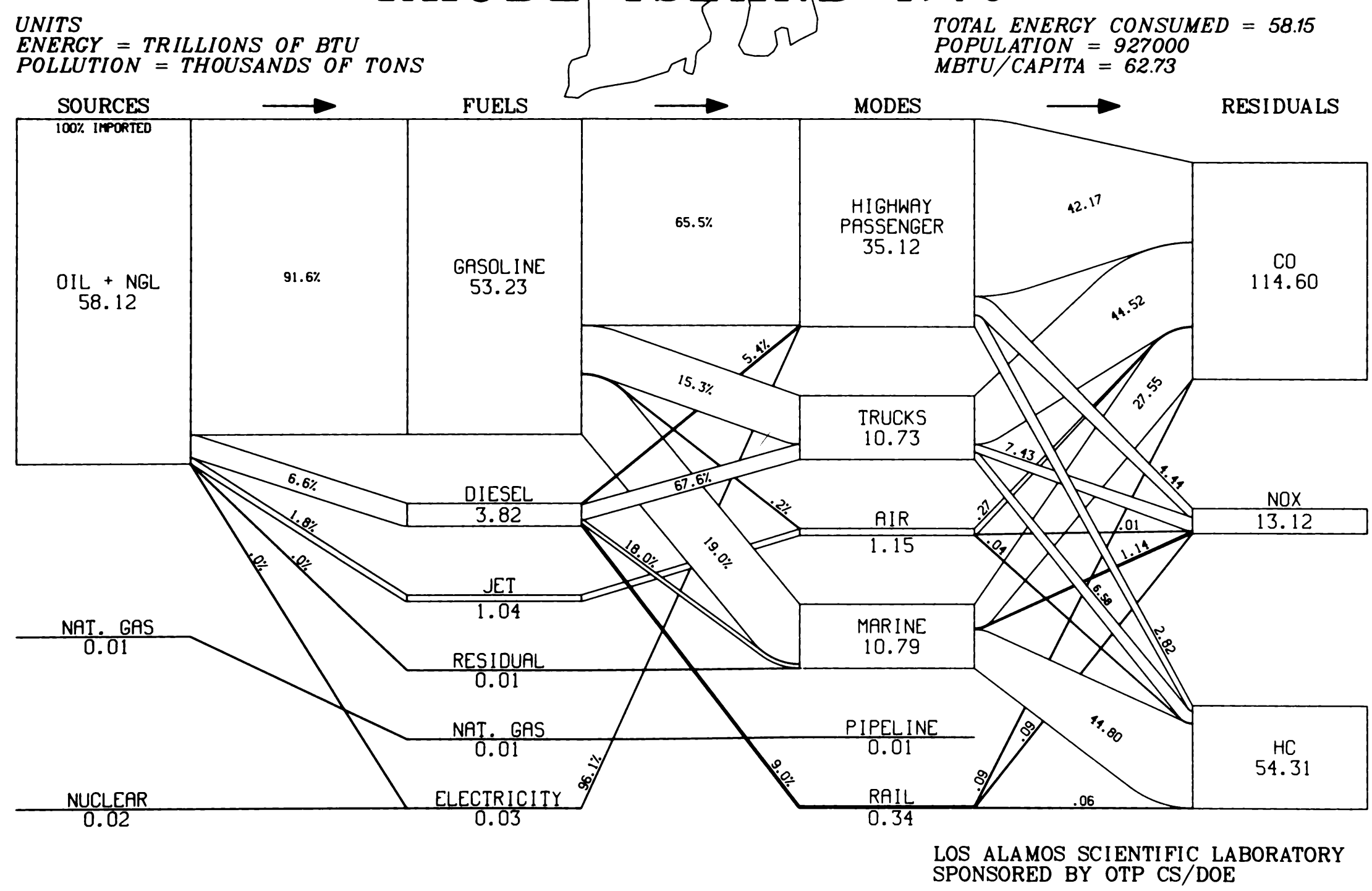




\section{TRANSPORTATION ENERGY FLOWS SOUTH CAROLINA 1976}

\section{UNITS}

ENERGY = TRILLIONS OF BTU

POLLUTION = THOUSANDS OF TONS

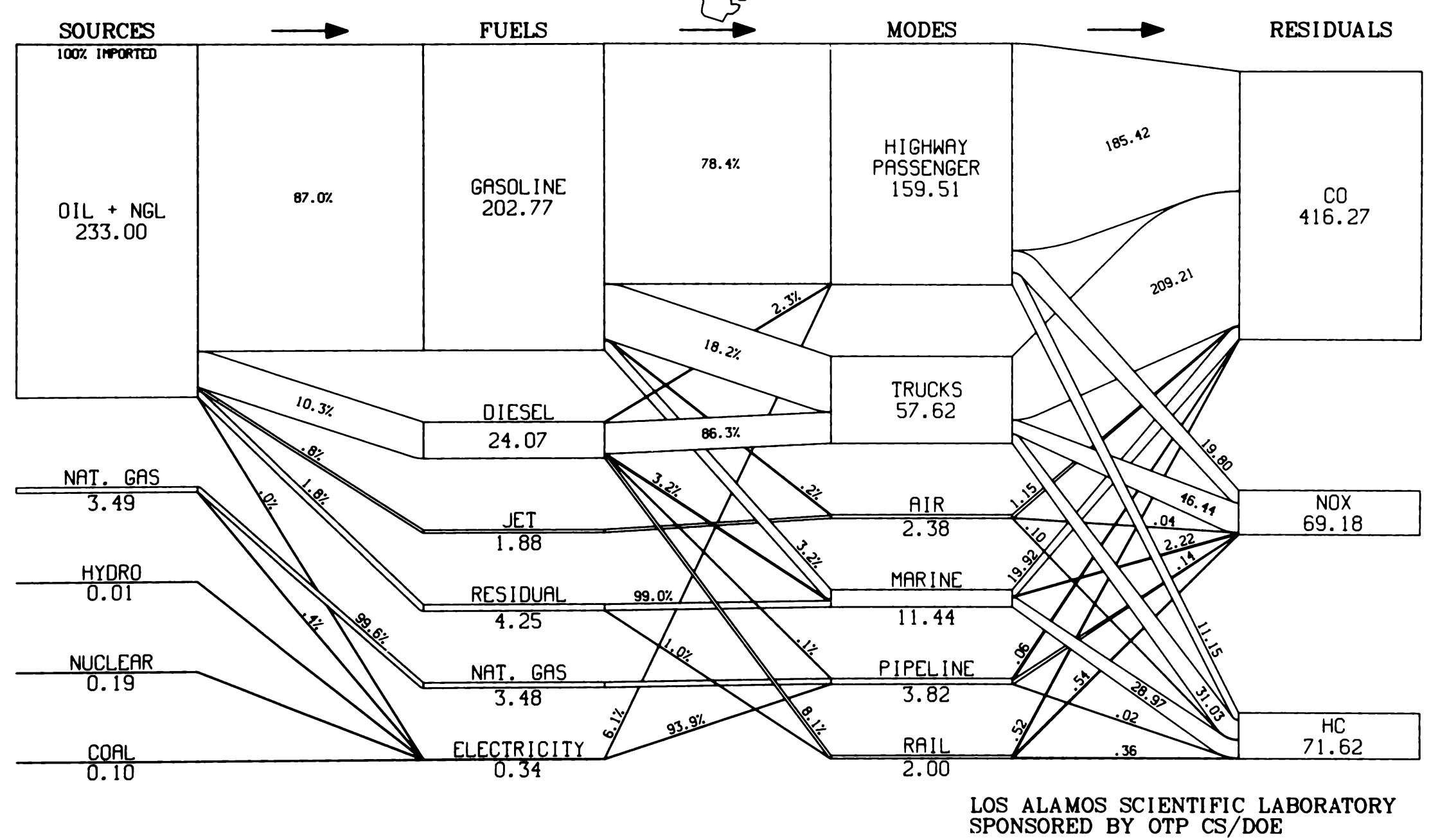

TOTAL ENERGY CONSUMED $=236.78$ POPULATION $=2767000$ MBTU $/$ CAPITA $=85.57$ SPONSORED BY OTP CS/DOE 


\section{TRANSPORTATION ENERGY FLOWS SOUTH DAKOTA 1976}

\section{UNITS}

ENERGY = TRILLIONS OF BTU

POLLUTION = THOUSANDS OF TONS

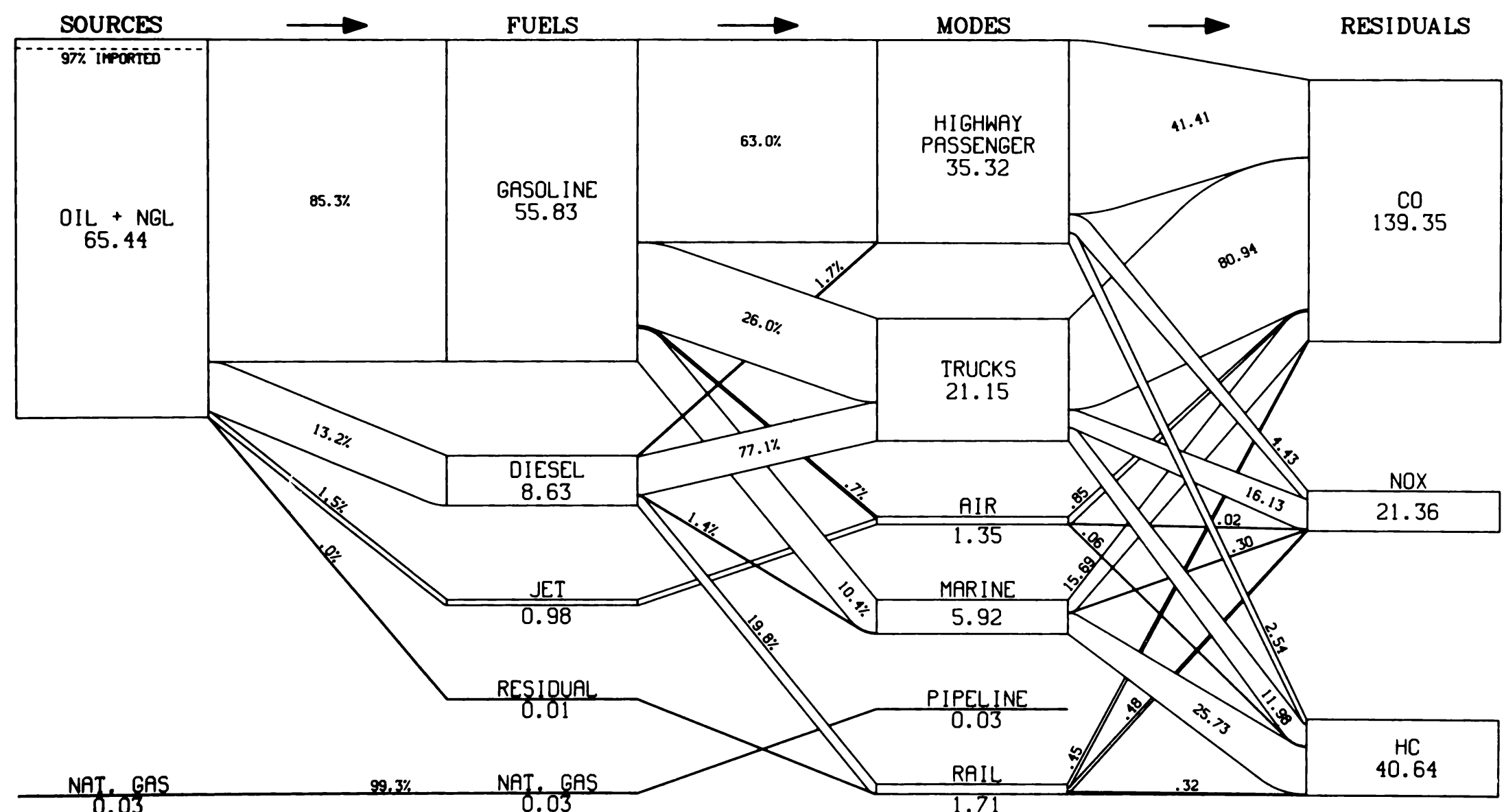

TOTAL ENERGY CONSUMED $=65.47$ POPULATION $=682000$

0.03 


\section{TRANSPORTATION ENERGY FLOWS TENNESSEE 1976}

UNITS
ENERGY = TRILLIONS OF BTU
POLLUTION $=$ THOUSANDS OF TONS

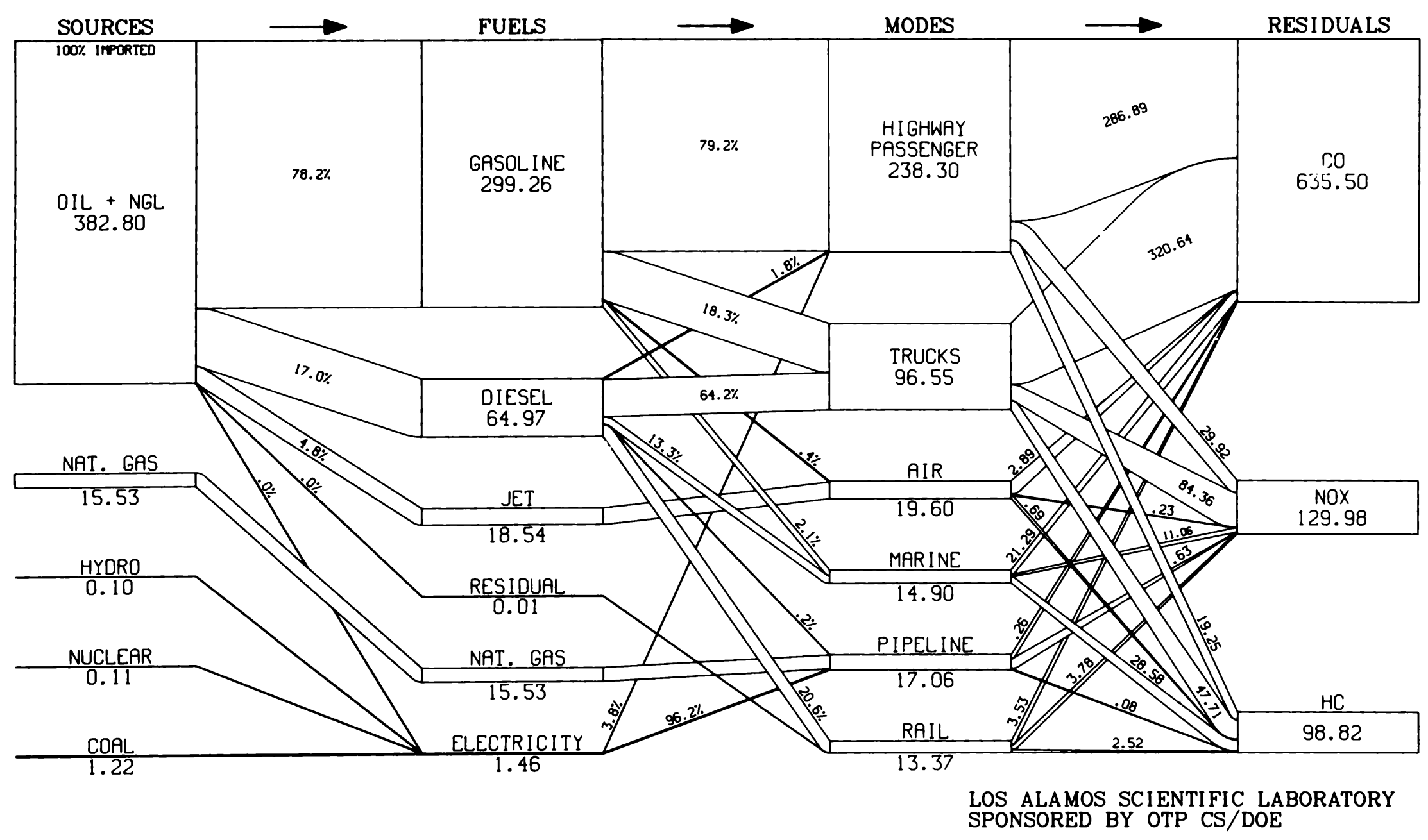

TOTAL ENERGY CONSUMED = 399.77 POPULATION $=4211000$ $M B T U / C A P I T A=94.94$ 


\section{TRANSPORTATION ENERGY FLOWS TEXAS 1976}

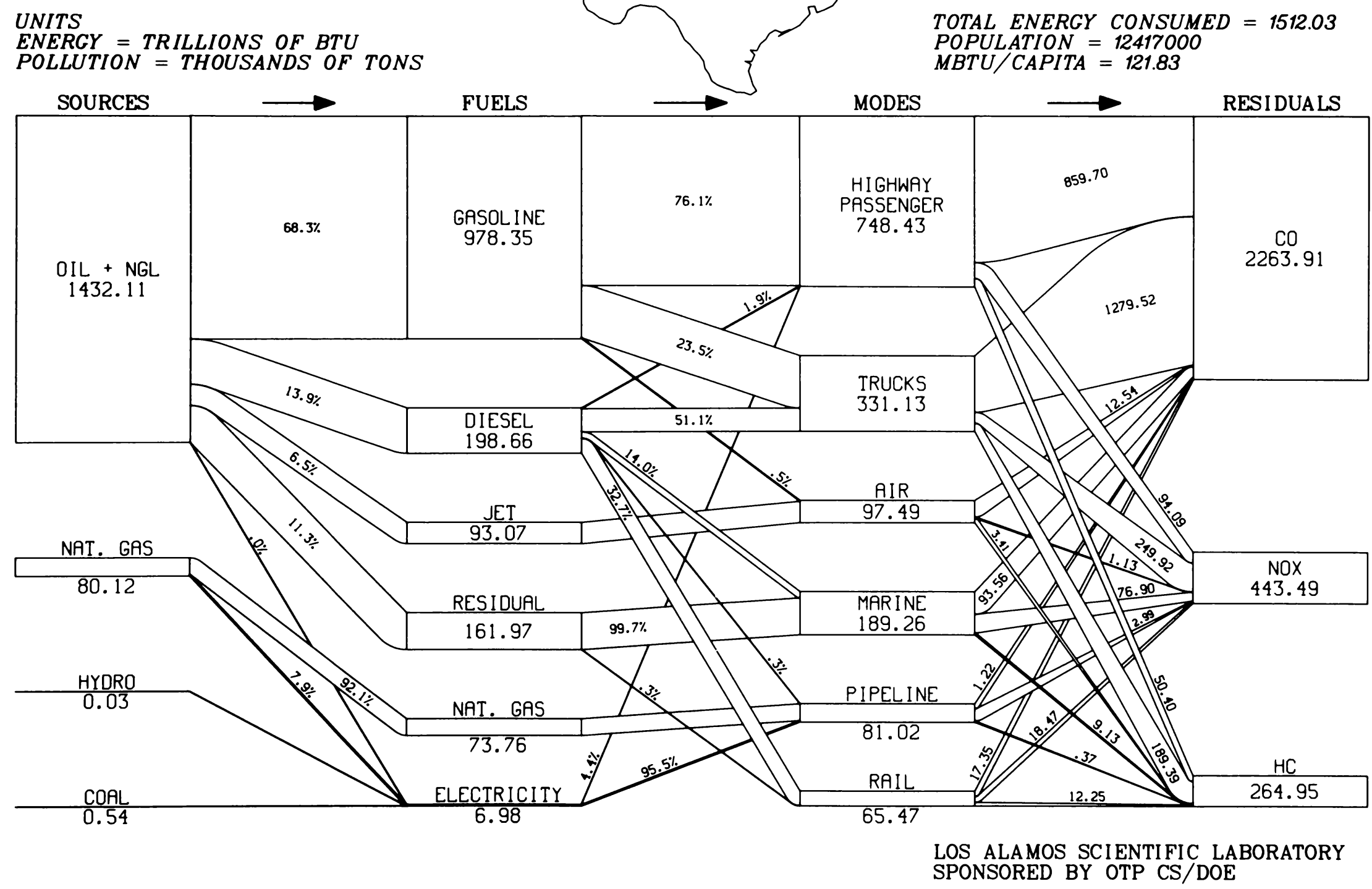




\section{TRANSPORTATION ENERGY FLOWS UT AH 1976}

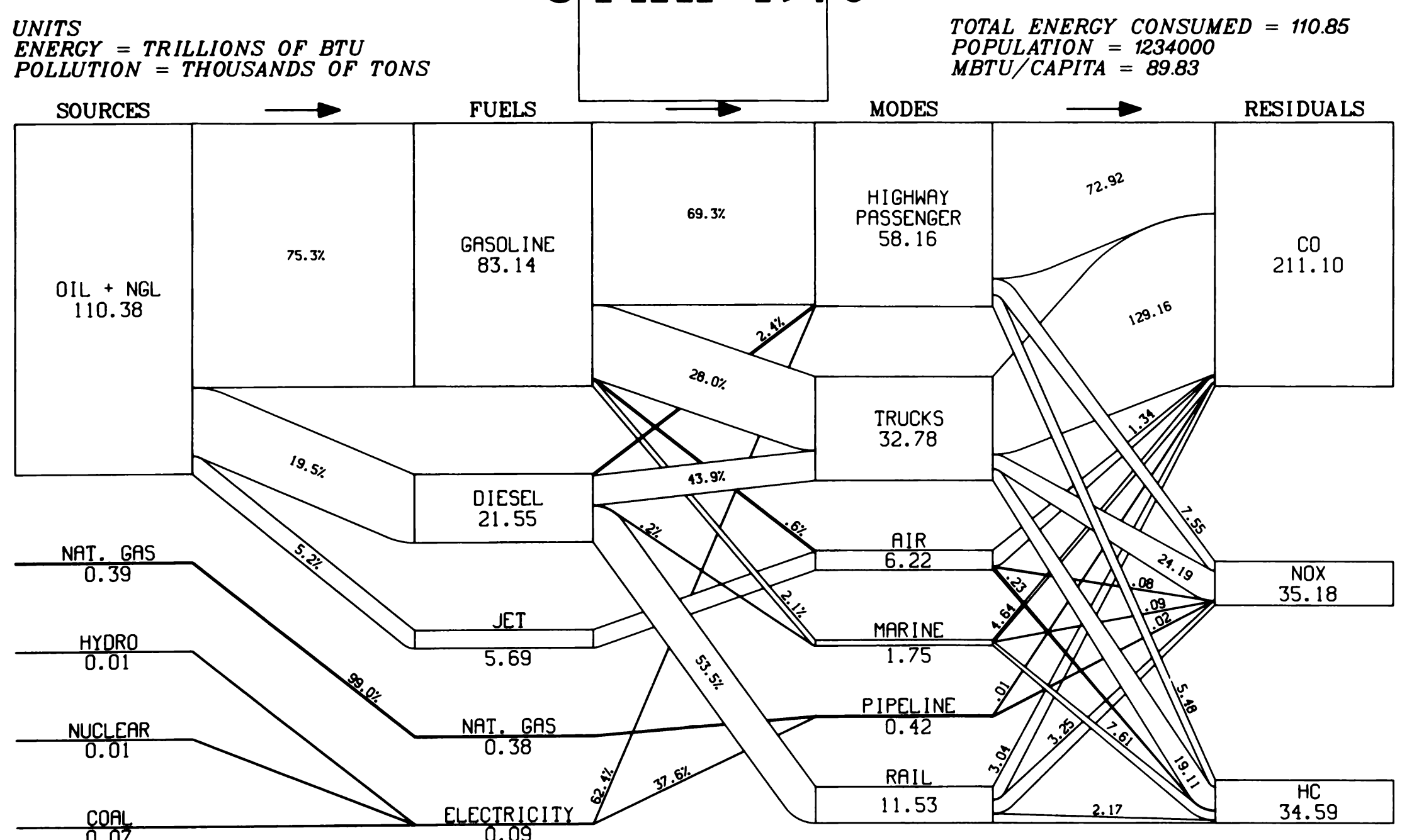




\section{TRANSPORTATION ENERGY FLOWS VERMONT 1976}

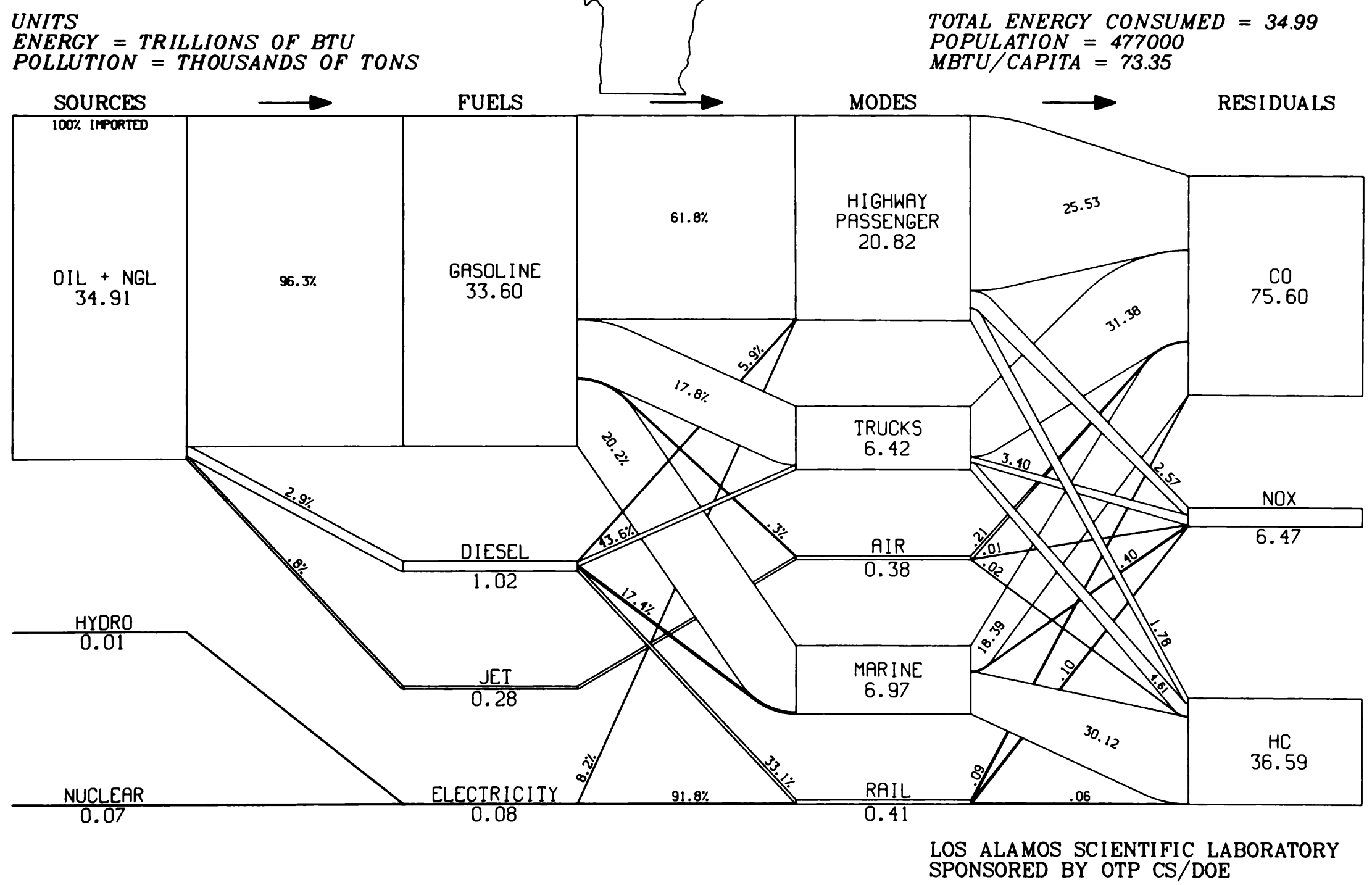




\section{TRANSPORTATION ENERGY FLOWS VIRGINIA 1\% \\ TOTAL ENERGY CONSUMED $=443.52$ POPULATION $=4906000$ MBTU/CAPITA $=90.40$}

UNITS $=$ TRILLIONS OF BTU POLLUTION = THOUSANDS OF TONS

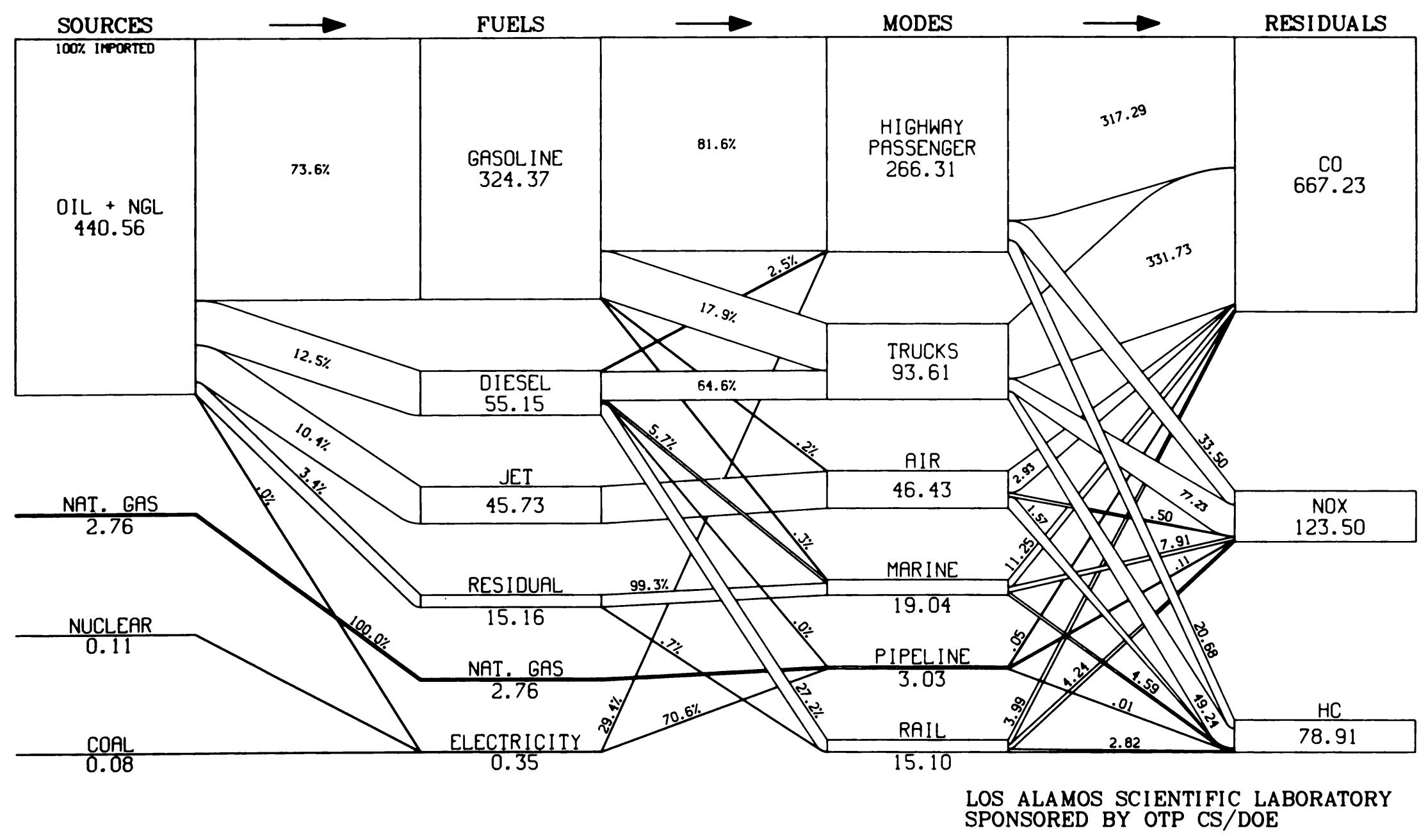




\section{TRANSPORTAGTON ENERGY FLOWS WASHINGTON 1976 \\ TOTAL ENERGY CONSUMED $=348.89$ POPULATION $=3570000$ $M B T U / C A P I T A=97.73$ \&

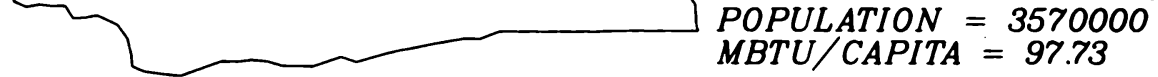

UNITS

ENERCY = TRILLIONS OF BTU

POLLUTION = THOUSANDS OF TONS

SOURCES

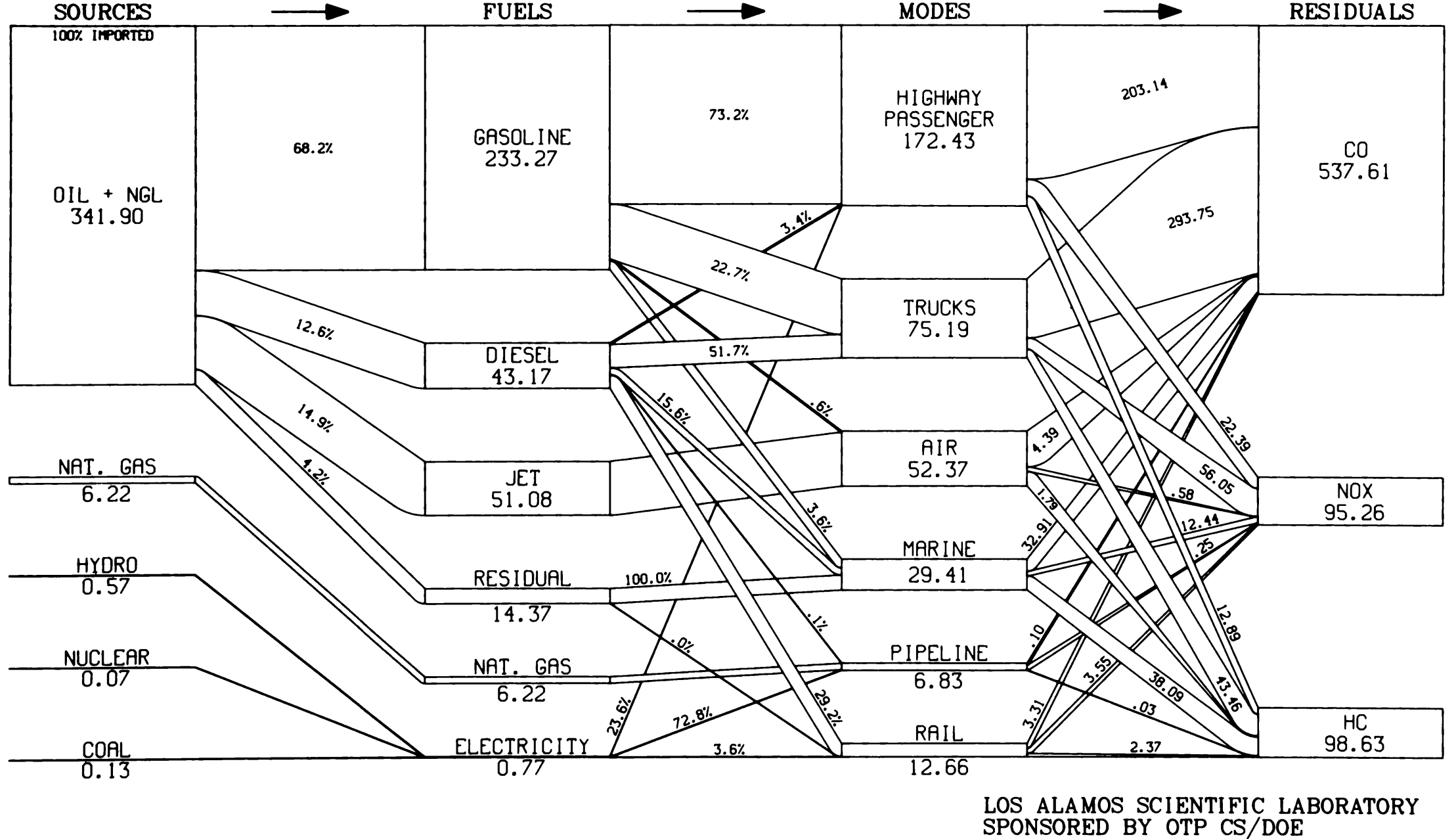




\section{TRANSPORTATION ENERGY FLOWS WEST VIRGINIA 1976}

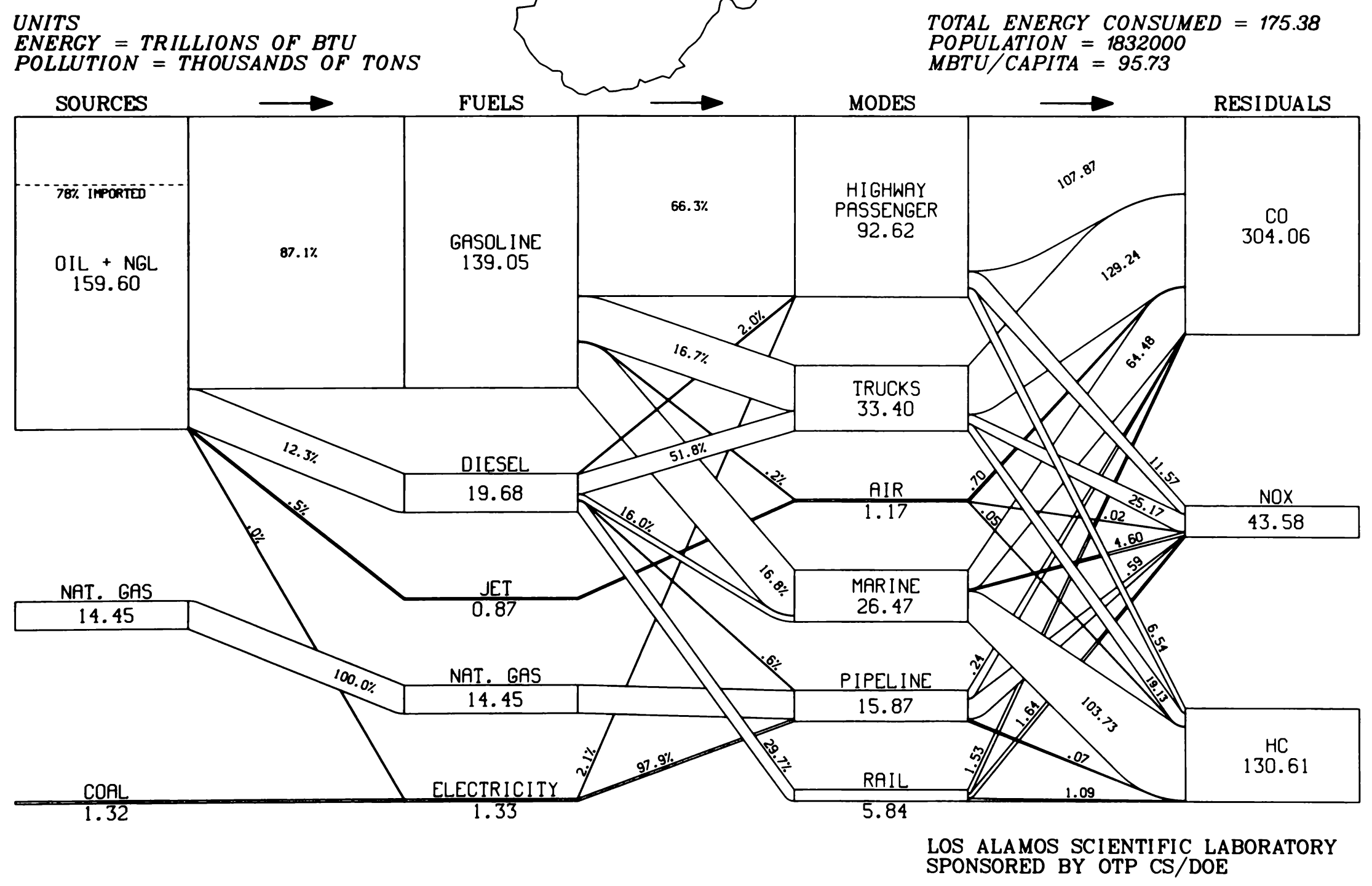




\section{TRANSPORTATION ENERGY FLOWS WISCONSIN 1976}

UNITS

ENERCY = TRILLIONS OF BTU

POLLUTION = THOUSANDS OF TONS

SOURCES

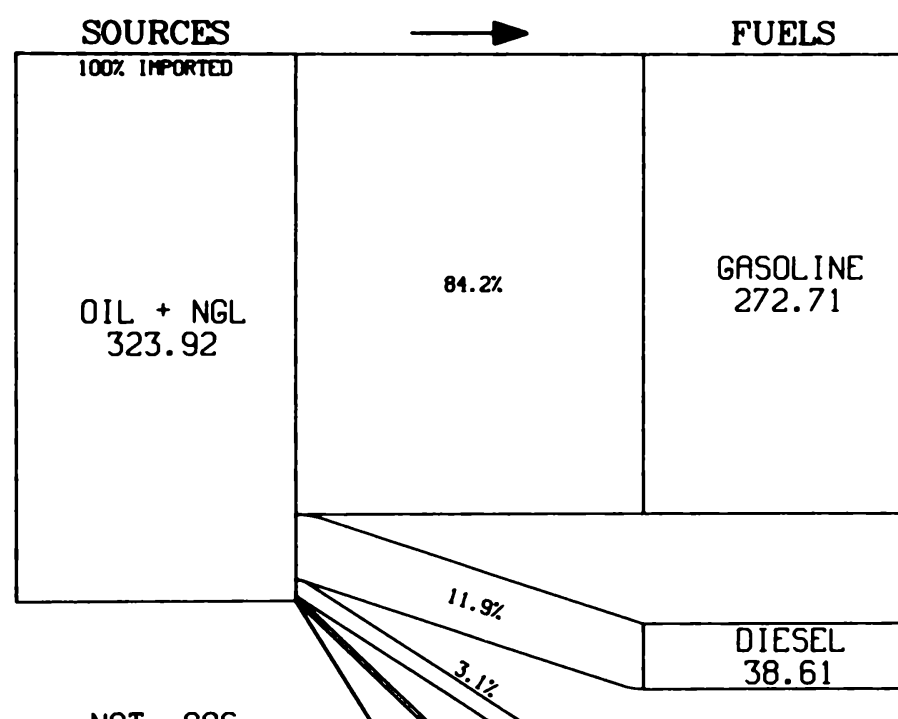

TOTAL ENERGY CONSUMED $=326.61$ POPULATION $=4621000$ $M B T U / C A P I T A=70.68$
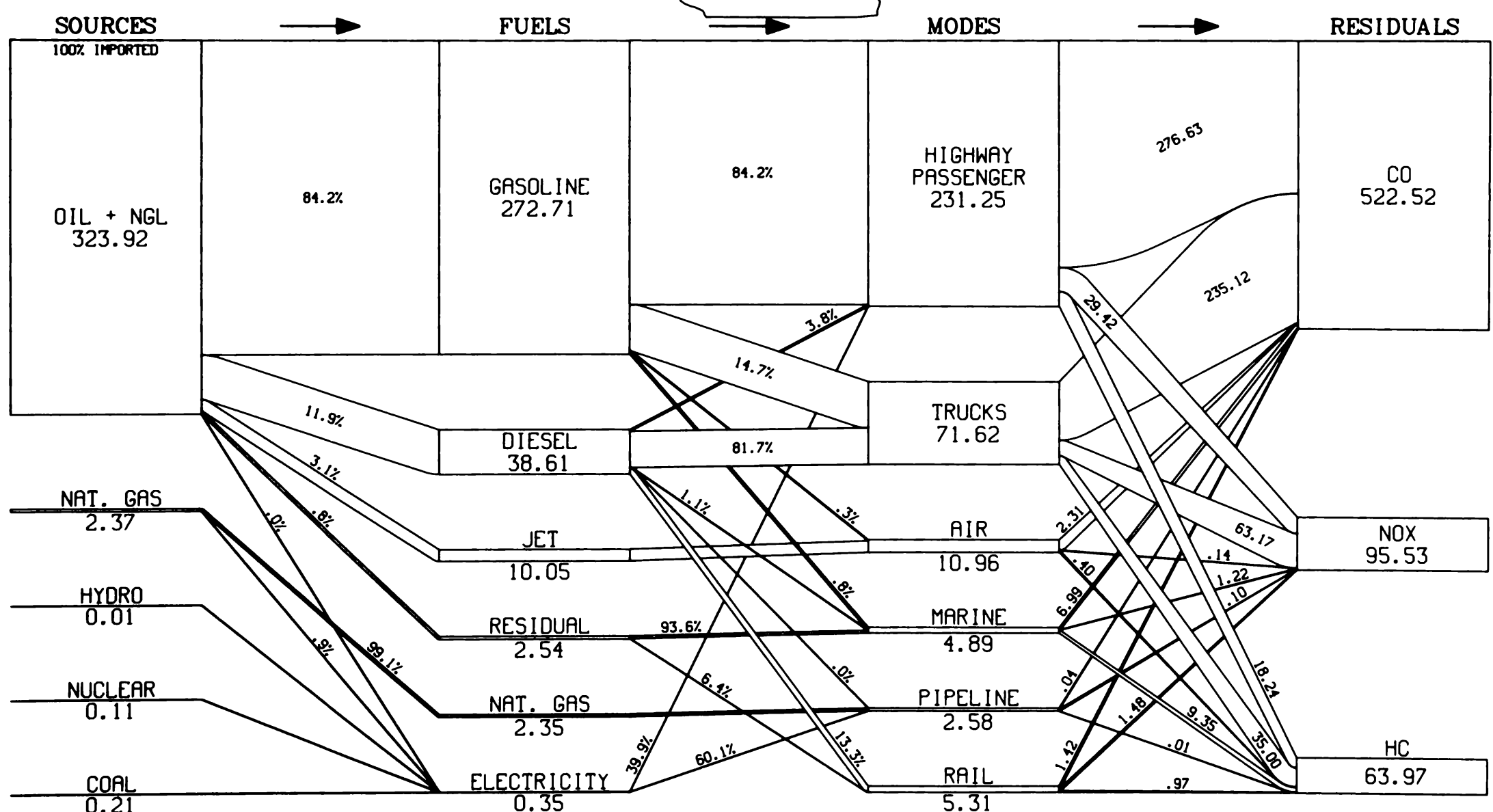

LOS ALAMOS SCIENTIFIC LABORATORY SPONSORED BY OTP CS/DOE 


\section{TRANSPORTATION ENERGY FLOWS WYOMING 1976}

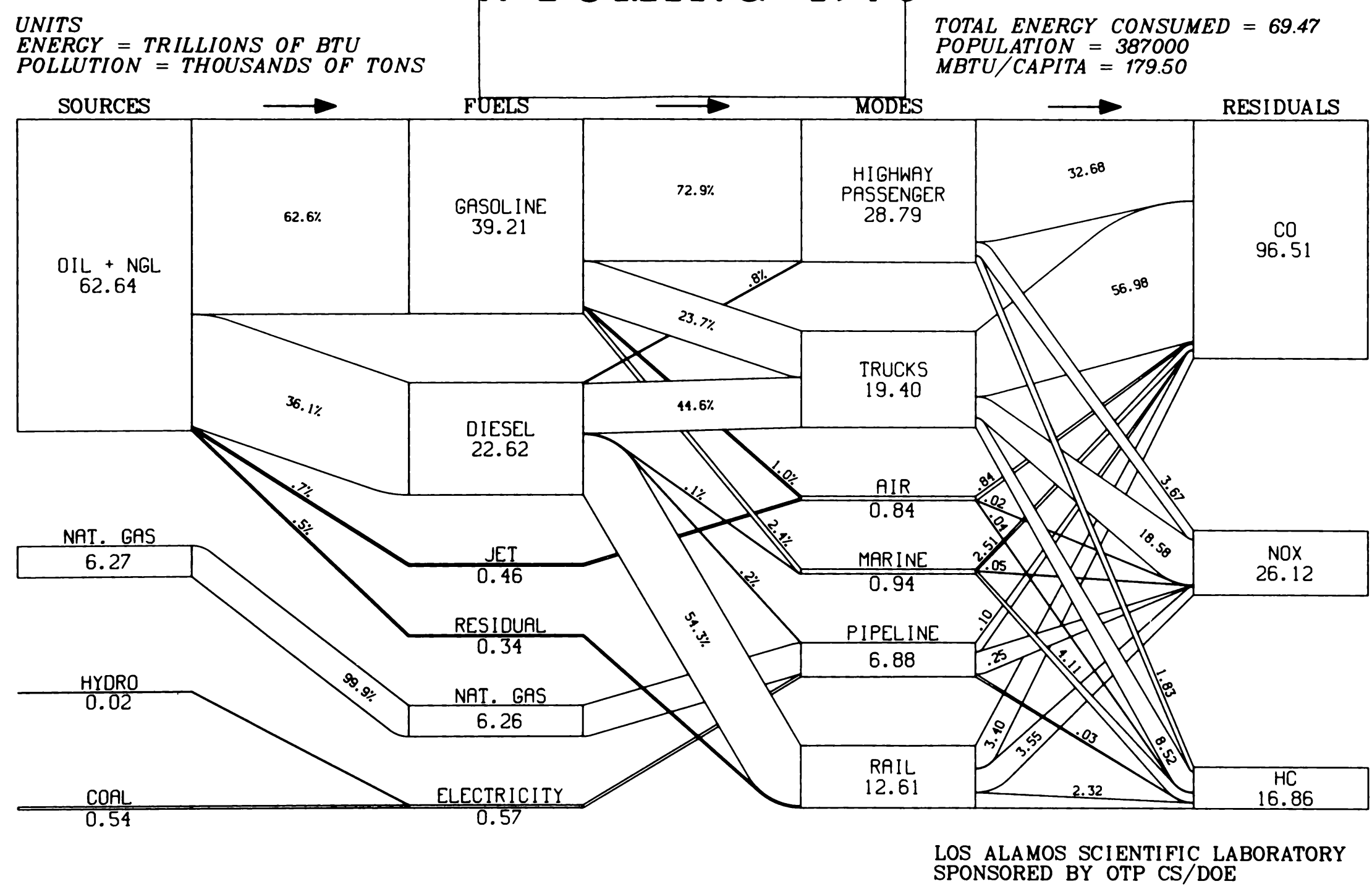




\section{TRANSPORTATION ENERGY FLOWS REGIONII 1976}

UNITS

ENERGY = TRILLIONS OF BTU

POLLUTION = THOUSANDS OF TONS

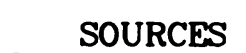

SOURCES

$100 \%$ If

OIL + NGL

843.08
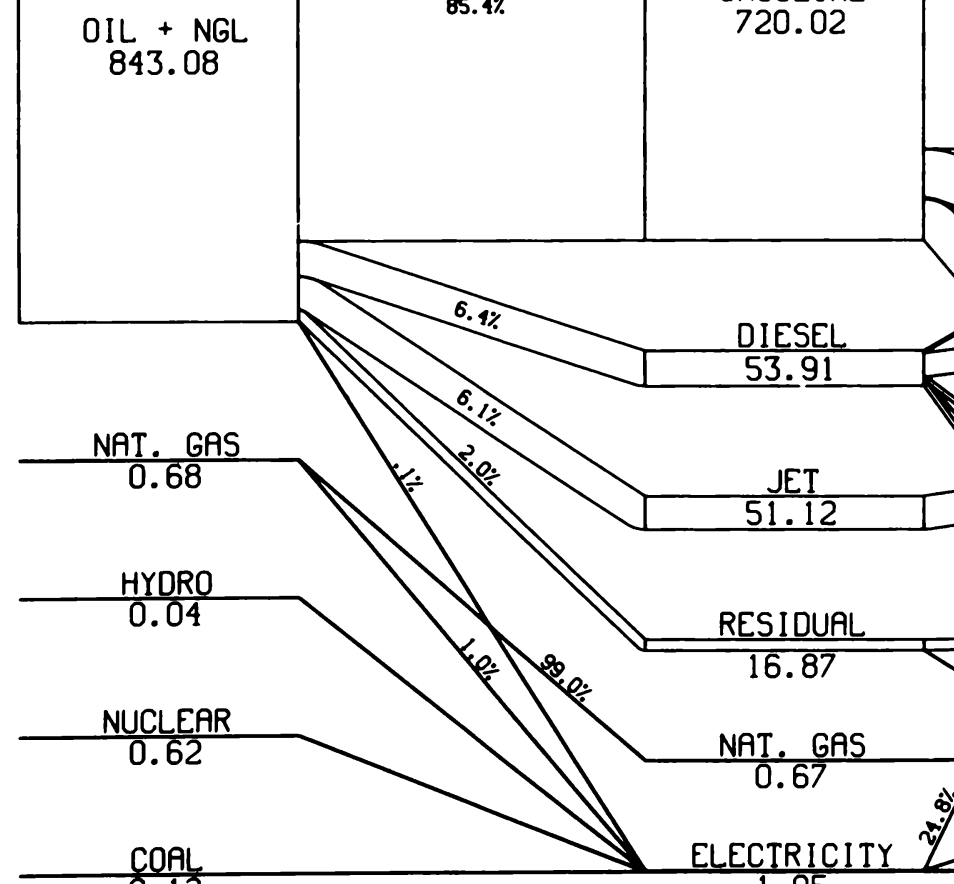

0.12

TOTAL ENERGY CONSUMED $=844.54$ POPULATION $=12135000$ $M B T U / C A P I T A=69.60$
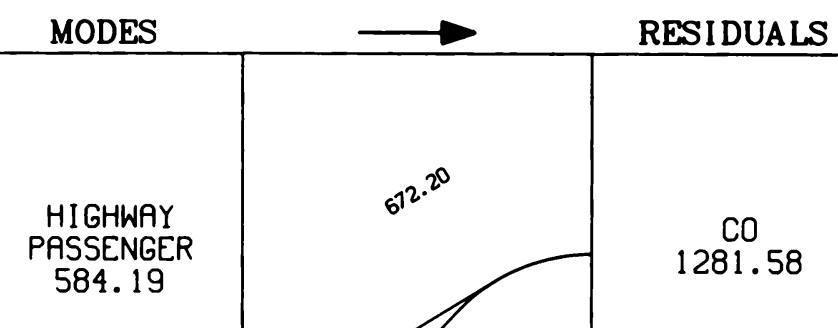


\section{TRANSPORTATION ENERGY FLOWS REGION II 1976}

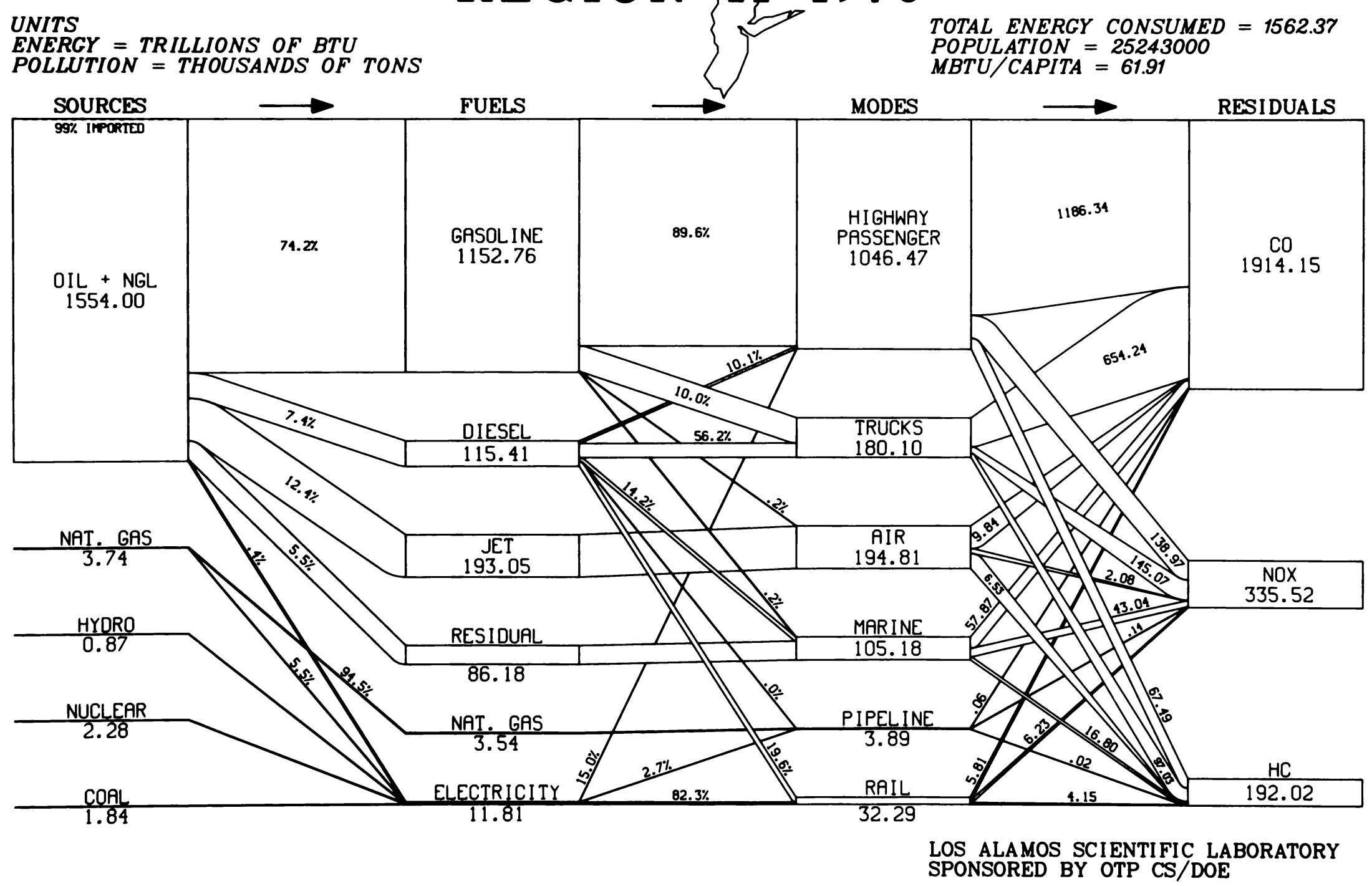




\section{TRANSPORTATION ENERGY FLOWS REGION III 1976}

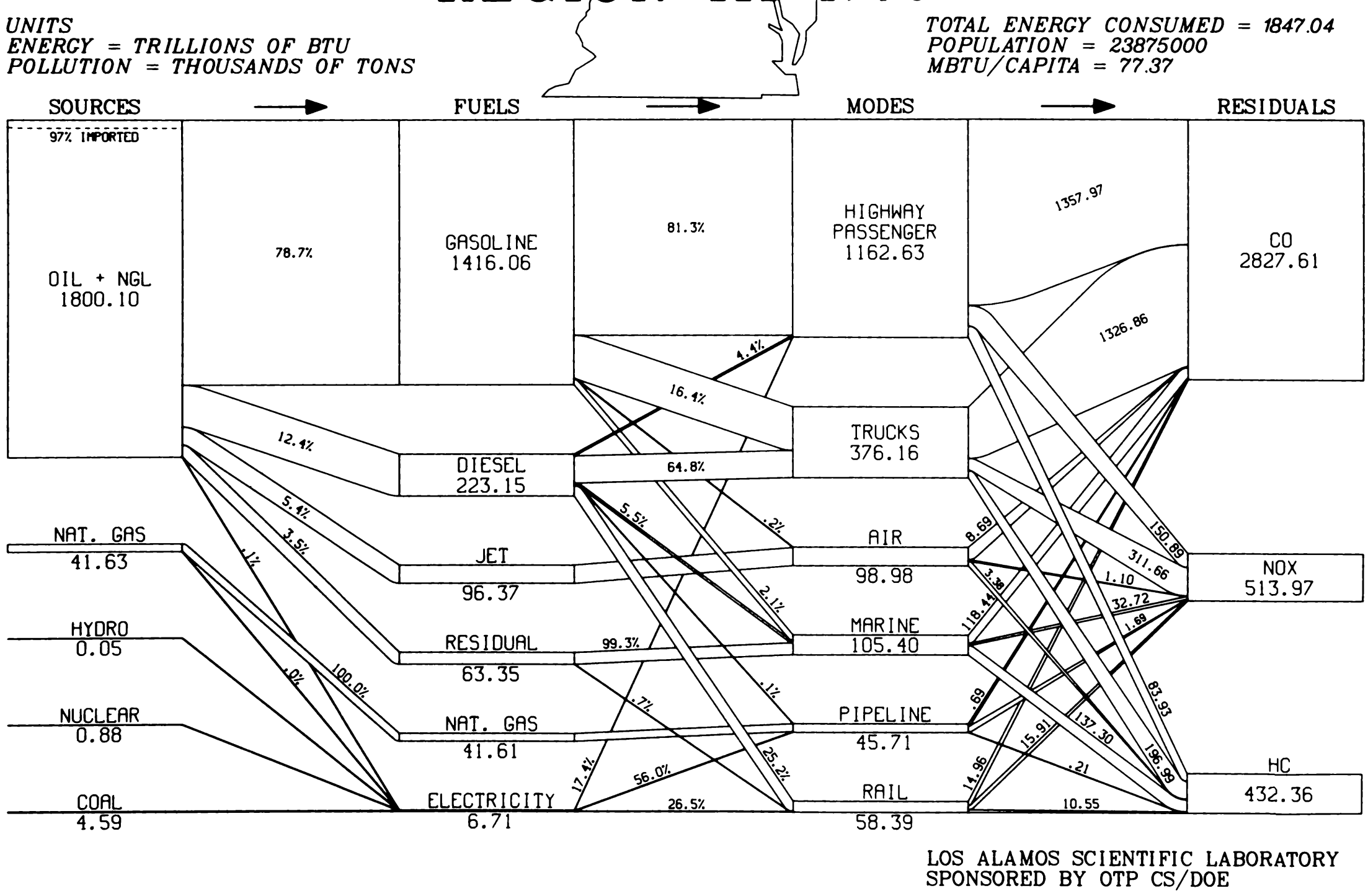




\section{TRANSPORTATION ENERGY FLOWS REGIQN IV 1976}

UNITS

ENERGY = TRILLIONS OF BTU

POLLUTION = THOUSANDS OF TONS



5.39

8.69

TOTAL ENERGY CONSUMED $=3189.80$

POPULATION $=34896000$

MBTU/CAPITA $=91.41$ 


\section{TRANSPORTATION ENERGY FLOWS REGION V 1976}

UNITS

ENERGY = TRILLIONS OF BTU POLLUTION = THOUSANDS OF TONS SOURCES

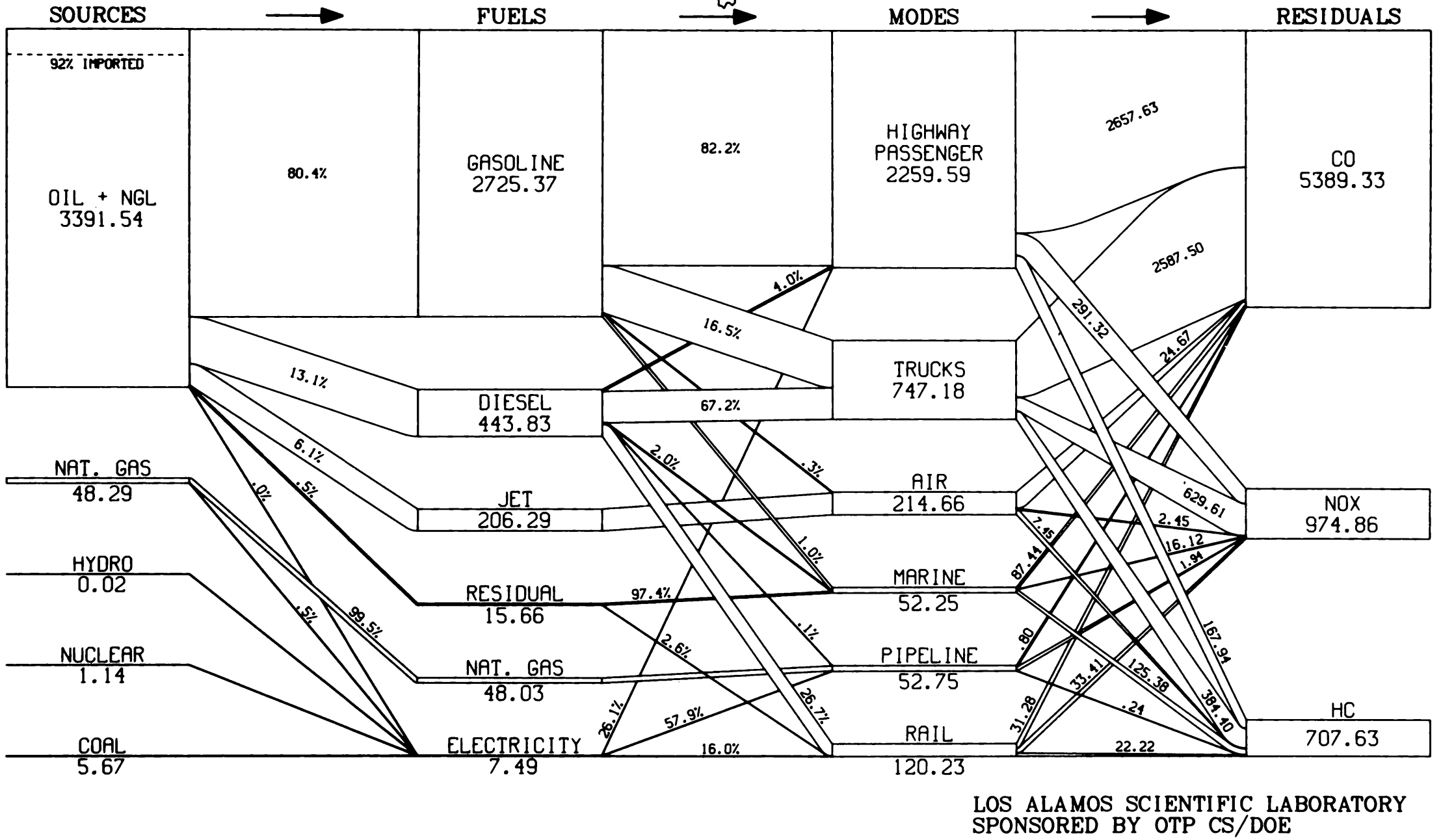

LOS ALAMOS SCIENTIFIC LABORATORY SPONSORED BY OTP CS/DOE

TOTAL ENERGY CONSUMED $=3446.57$ POPULATION $=44854000$ MBTU $/$ CAPITA $=76.84$

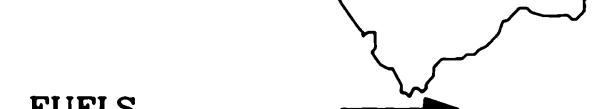

MODES 


\section{TRANSPORTATION ENERGY FLOWS REGION VI 1976}

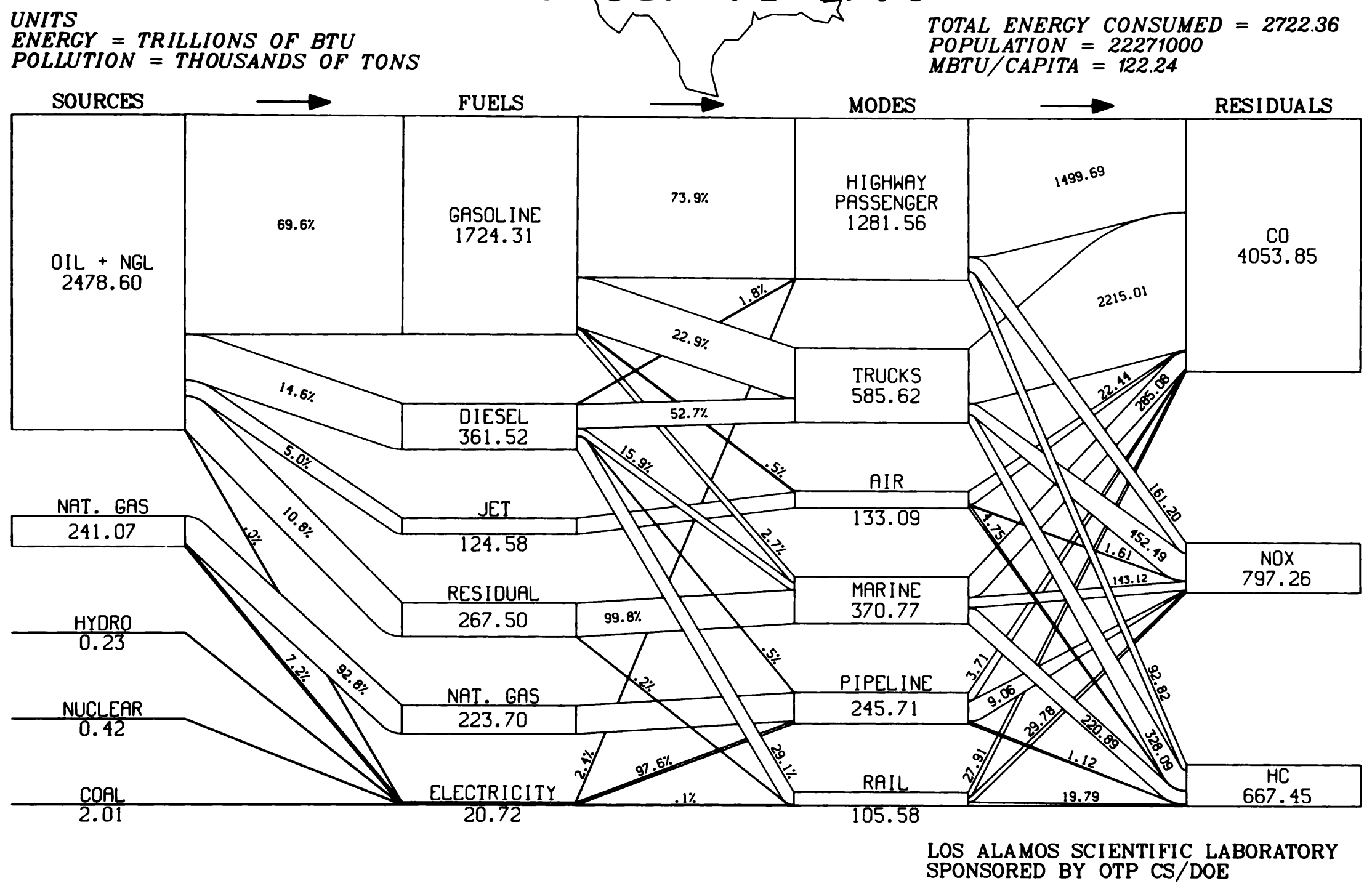




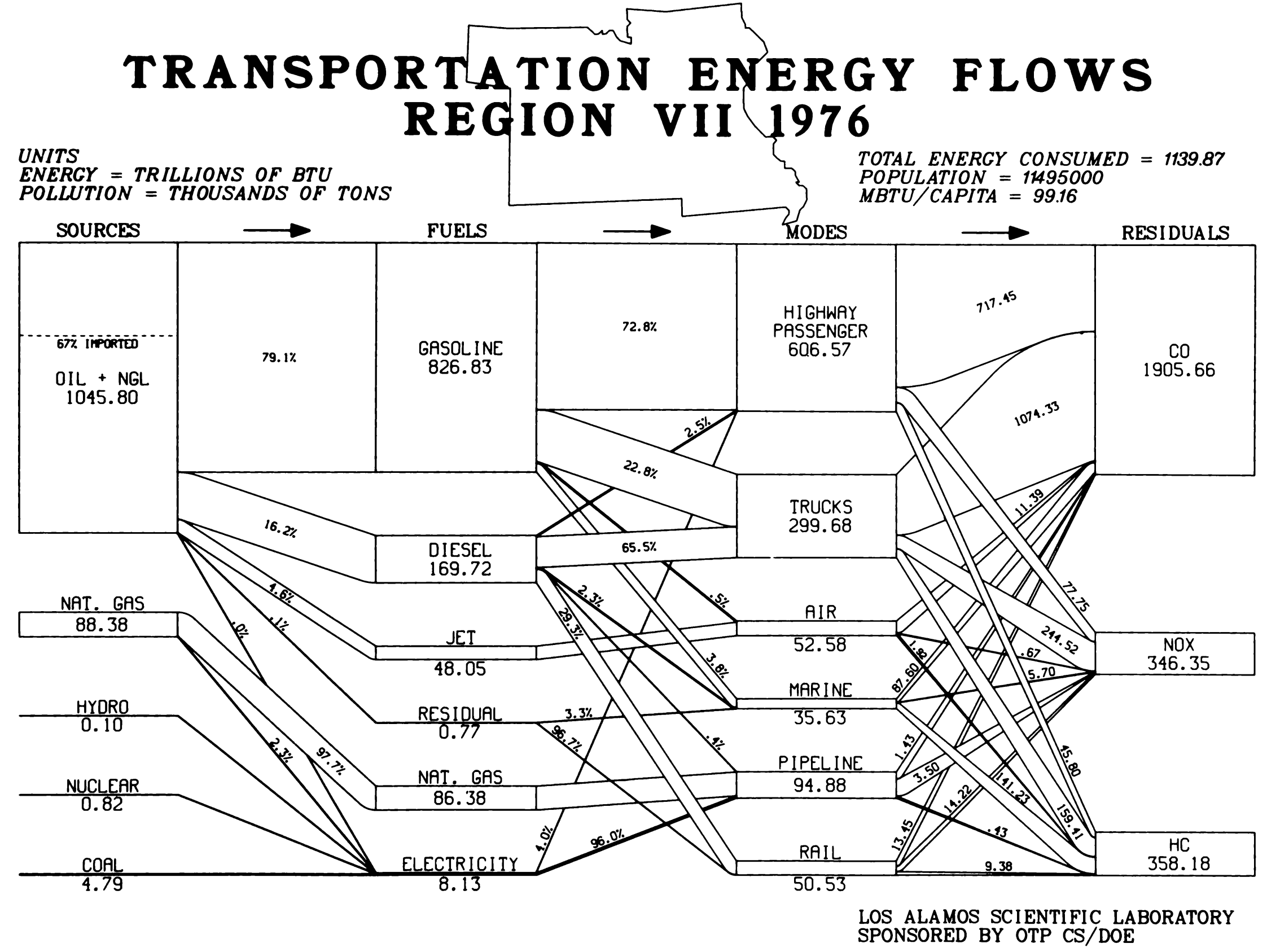




\section{TRANSPORTATION ENERGY FLOWS REGION VIII 1976}

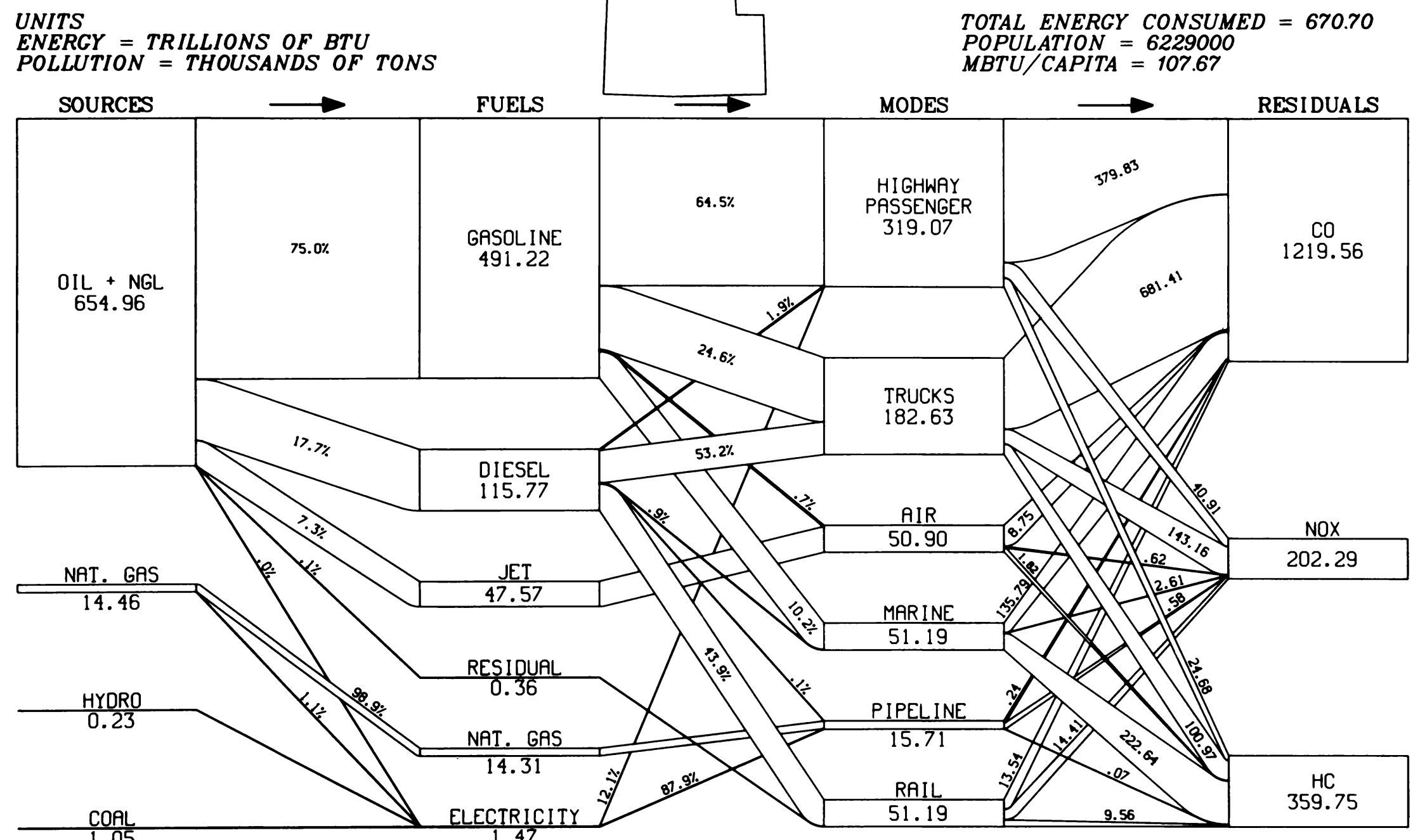




\section{TRANSPORTATION ENERGY FLOWS REGION IX 1976}

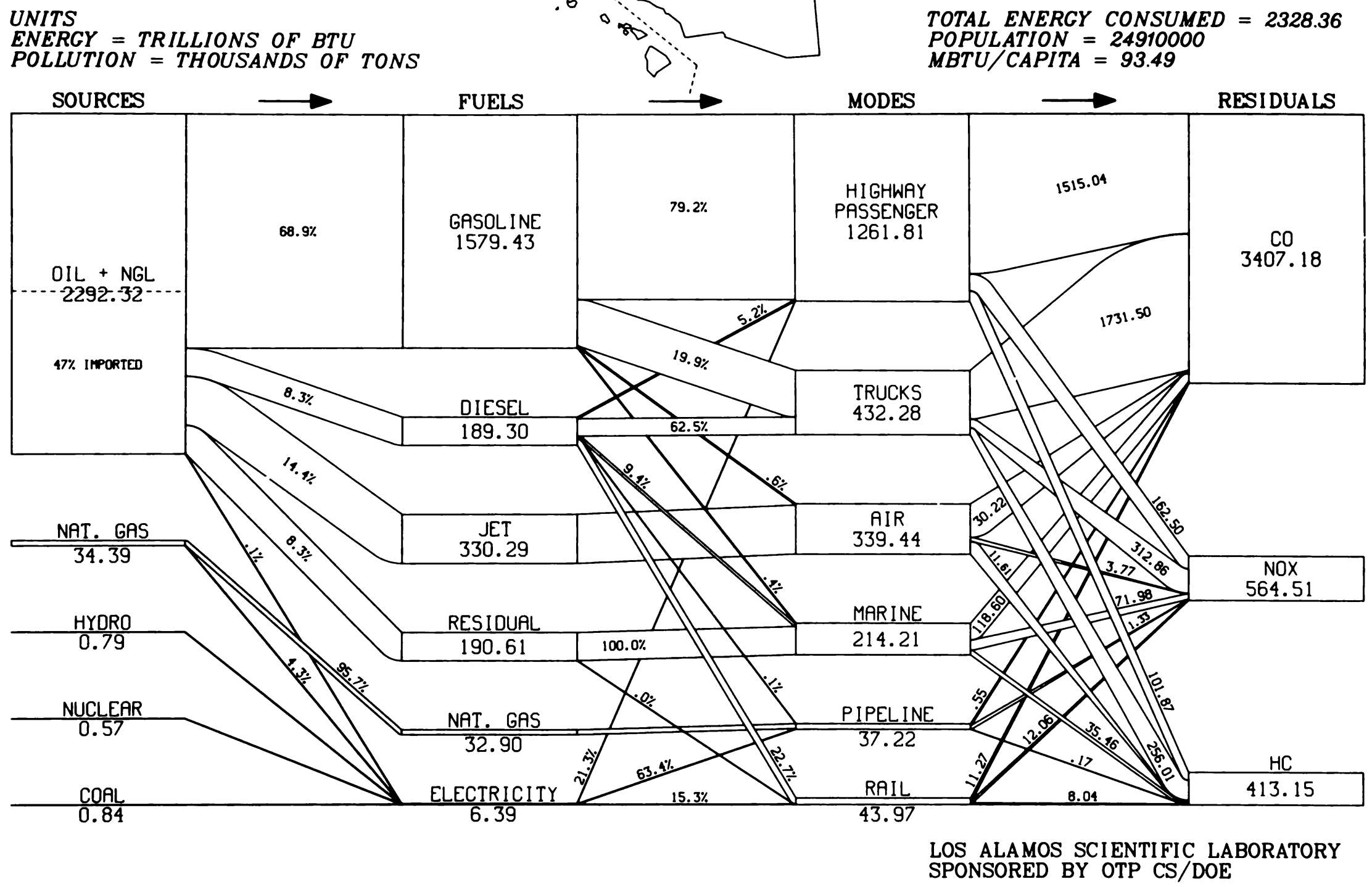




\section{TRANSPORTATION ENERGY FLOWS REGION $X 1976$}

\section{UNITS}

ENERGY = TRILLIONS OF BTU

POLLUTION = THOUSANDS OF TONS

SOURCES

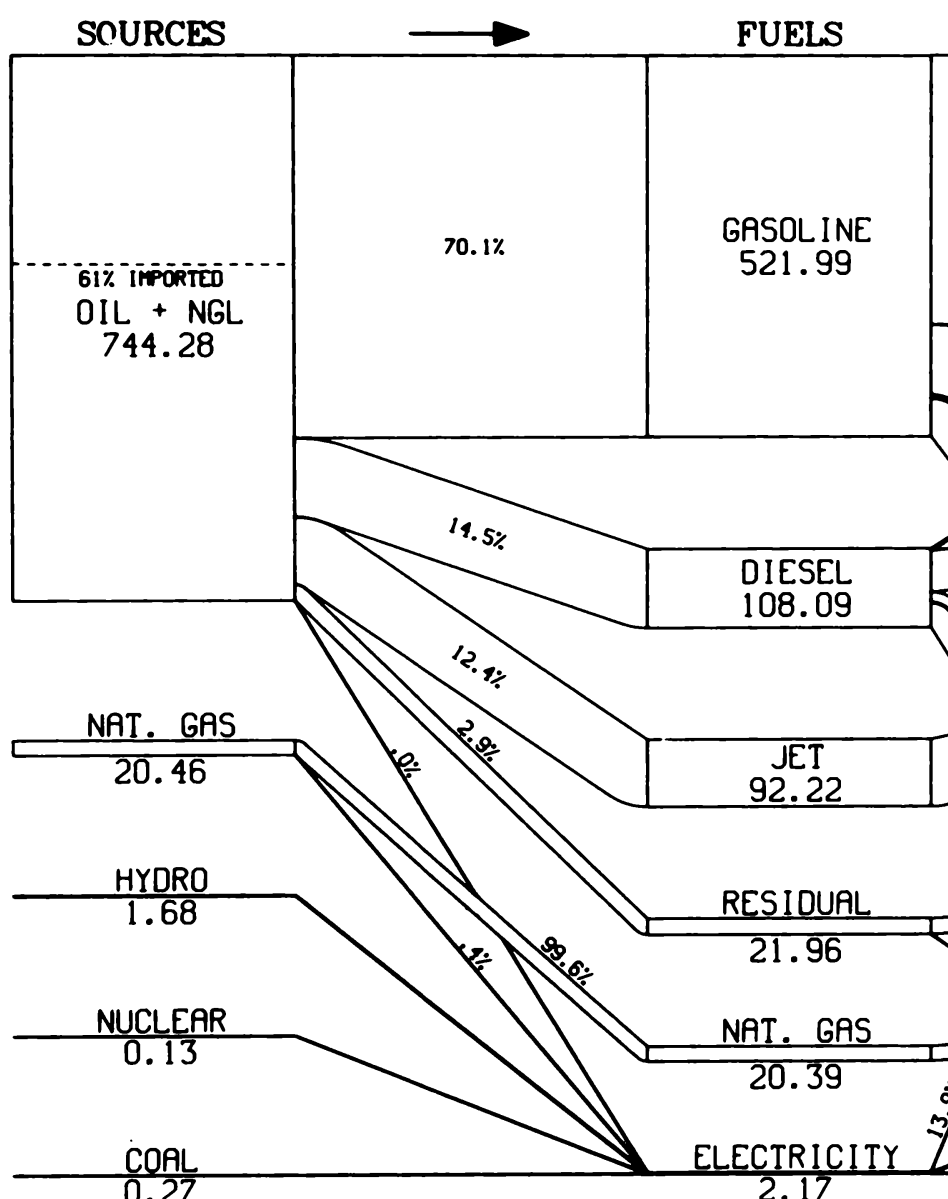

2.17

TOTAL ENERGY CONSUMED $=766.82$

POPULATION $=7107000$

MBTU $/$ CAPITA $=107.90$
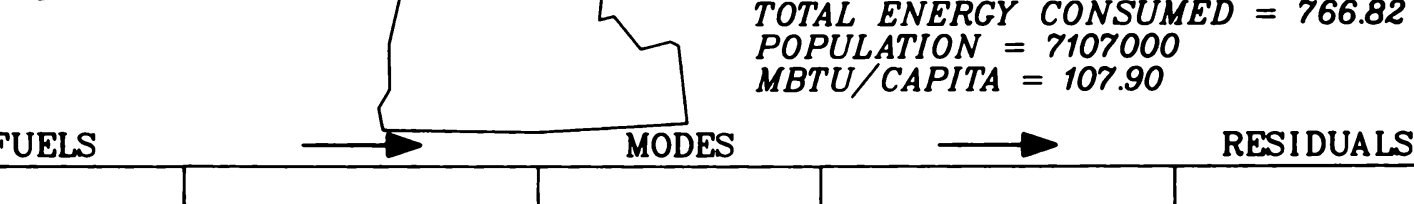

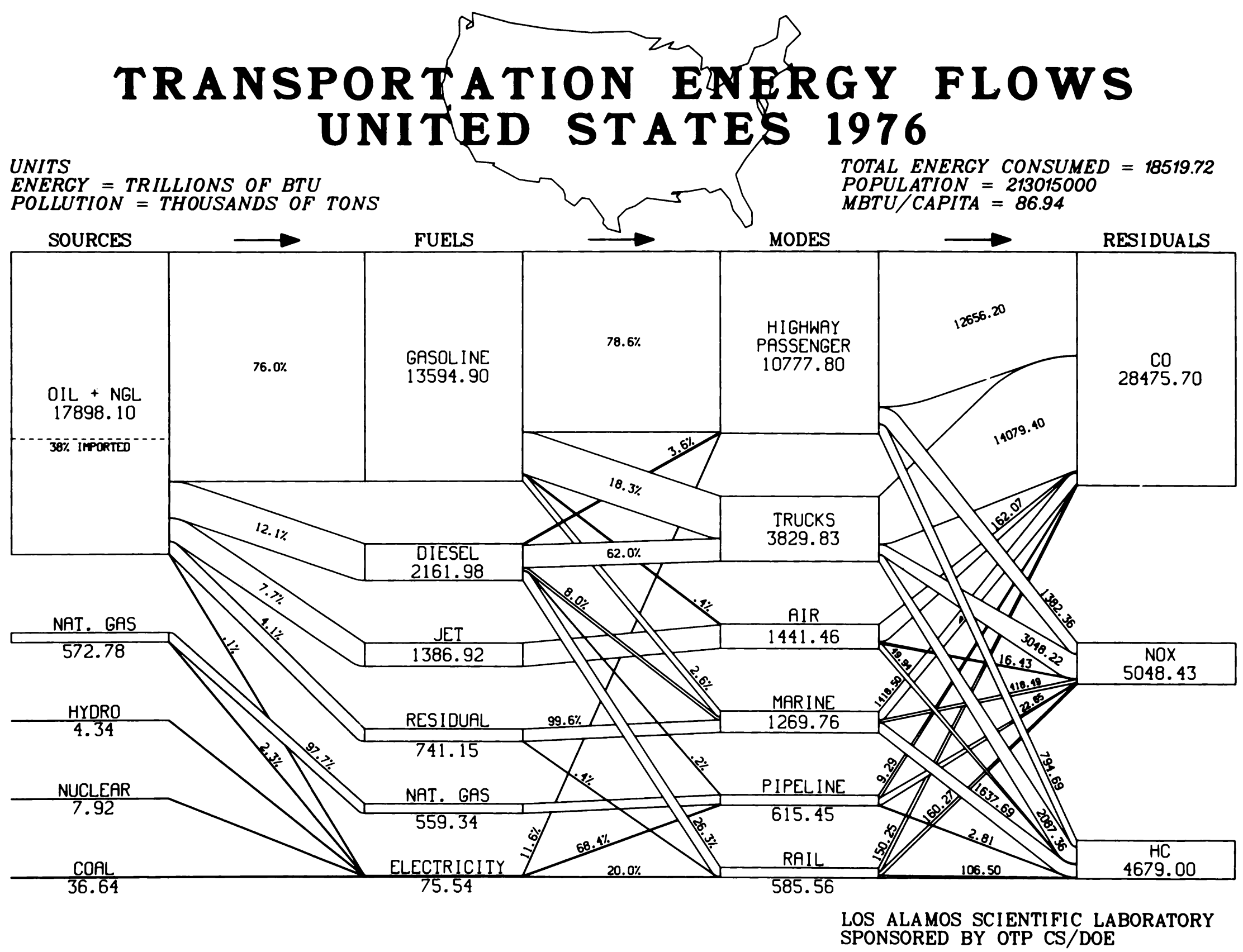


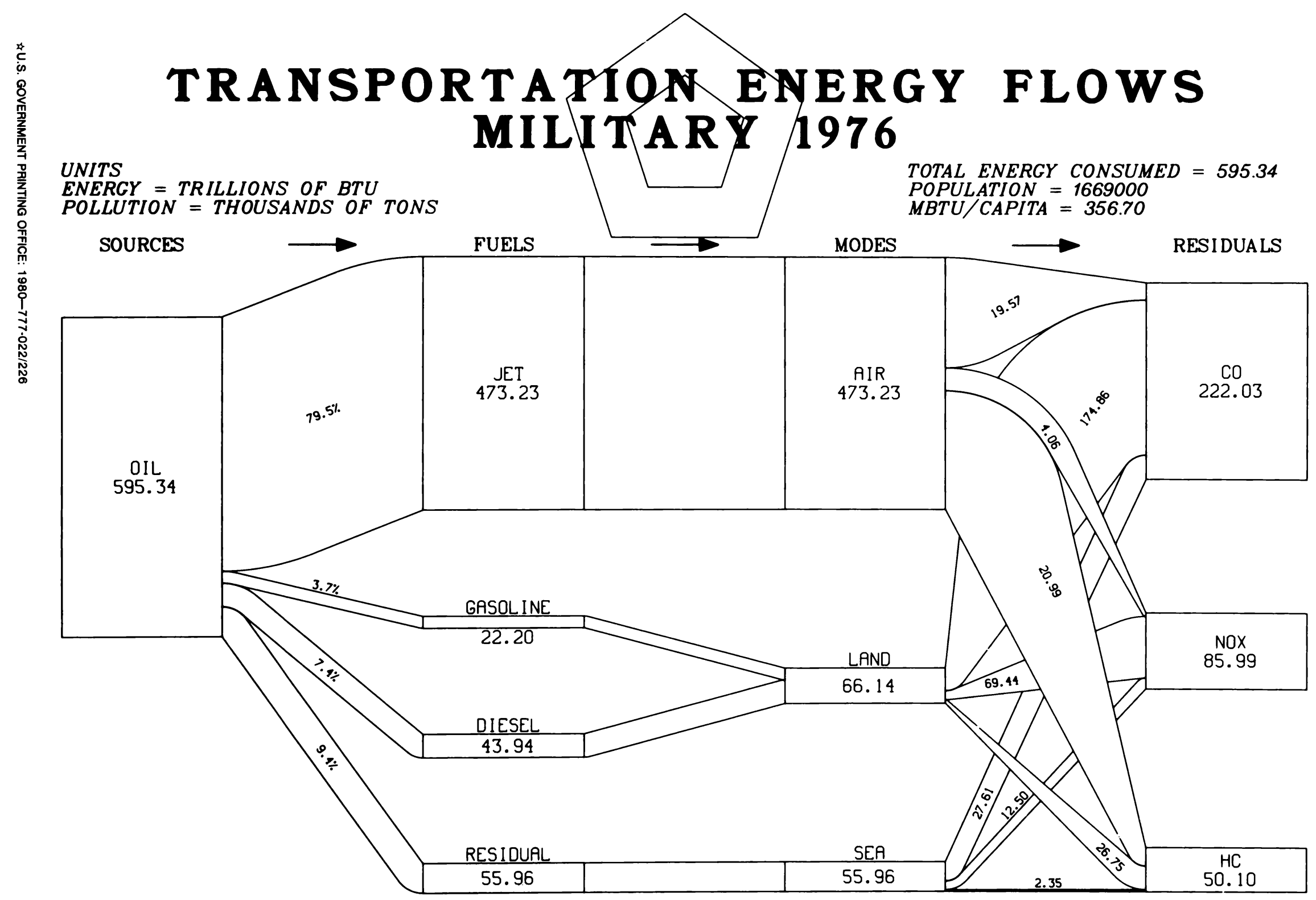

LOS ALAMOS SCIENTIFIC LABORATORY 



\section{TRANSPORTATION ENERGY FLOWS UNITED STATES 1976}

\section{UNITS}

ENERGY = TRILLIONS OF BTU

POLLUTION = THOUSANDS OF TONS

SOURCES

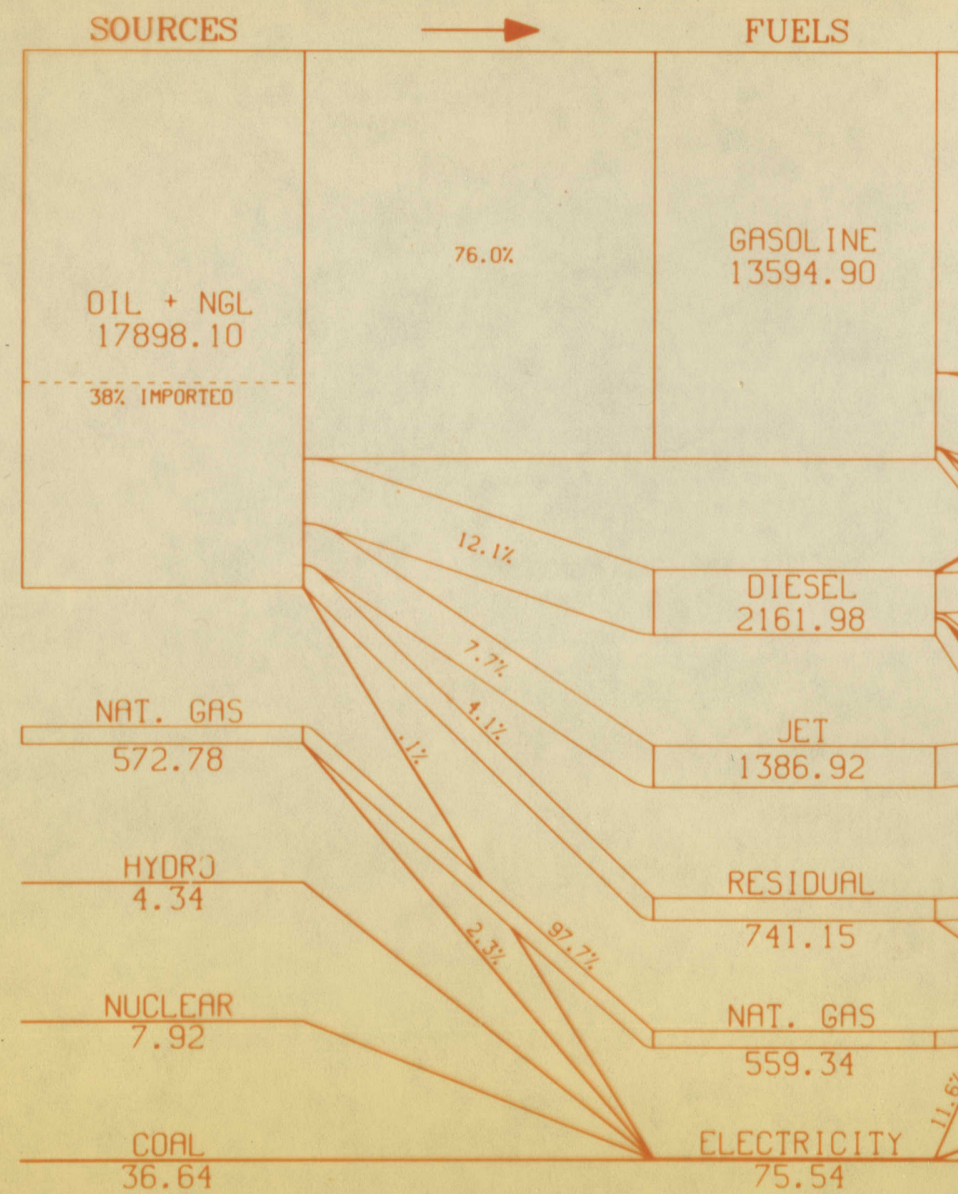

TOTAL ENERCY CONSUMED POPULATION $=213015000$

$M B T U / C A P I T A=86.94$

MODES

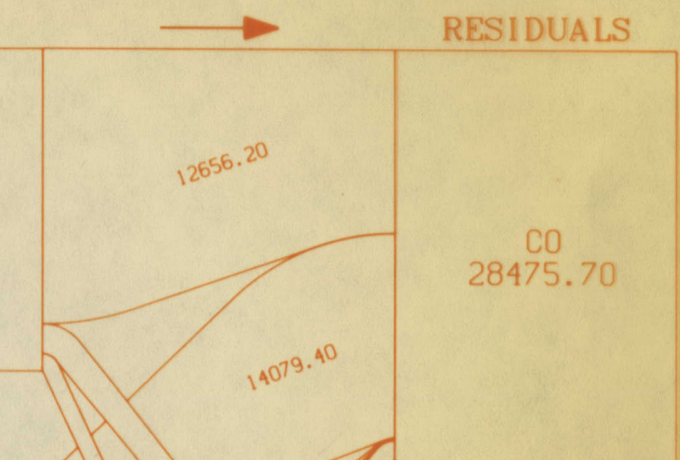





\section{TRANSPORTATION ENERGY FLOWS GEORGIA 1976}

\section{UNITS}

ENERCY = TRILLIONS OF BTU POLLUTION = THOUSANDS OF TONS

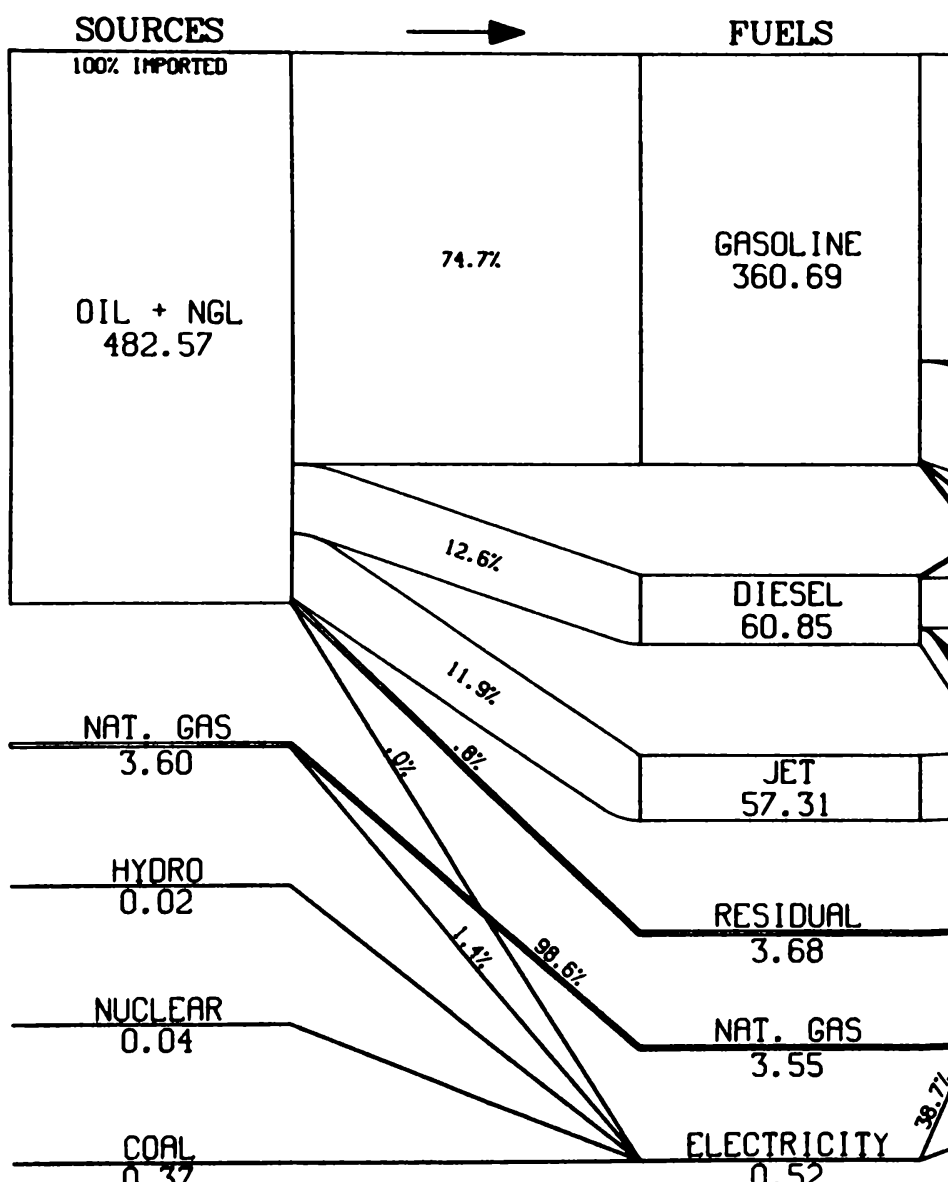

SOURCES

0.37

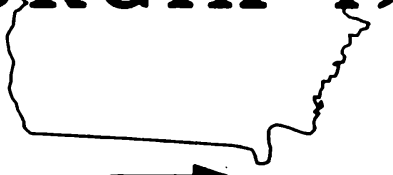

TOTAL ENERGY CONSUMED $=486.60$ POPULATION $=4910000$ MBTU $/$ CAPITA $=99.10$ MODES $\longrightarrow \quad$ RESIDUALS

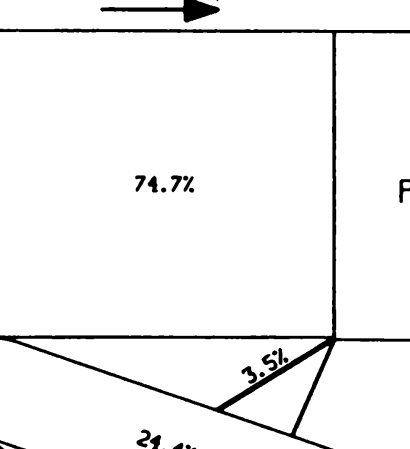

HIGHWAY

PASSENGER

271.61

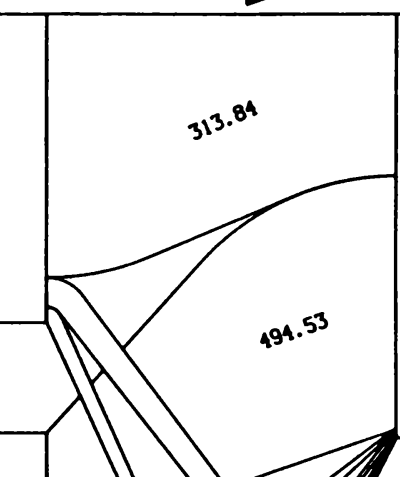
RESIDUALS

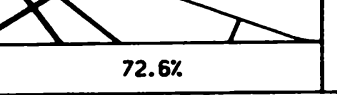

TRUCKS

132.14
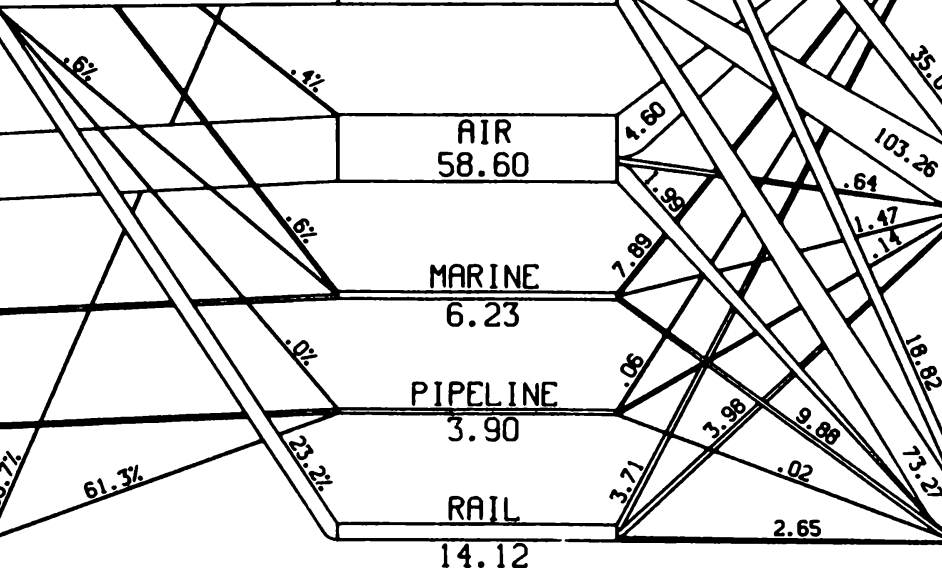

LOS ALAMOS SCIENTIFIC LABORATORY SPONSORED BY OTP CS/DOE 


\section{0 \\ TRANSPORTATION $\triangle$ ENERGY FLOWS HA W AII 1276}

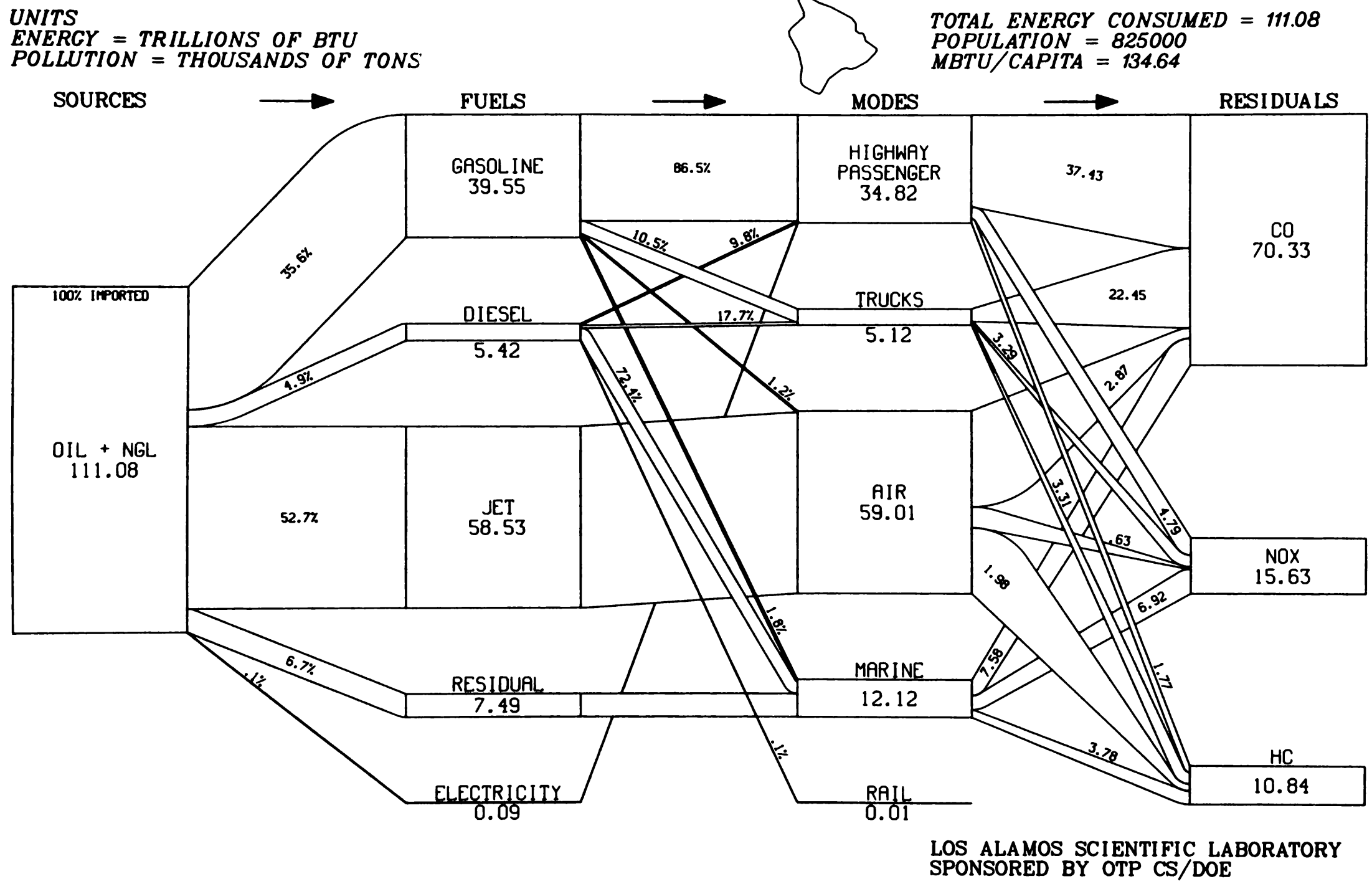




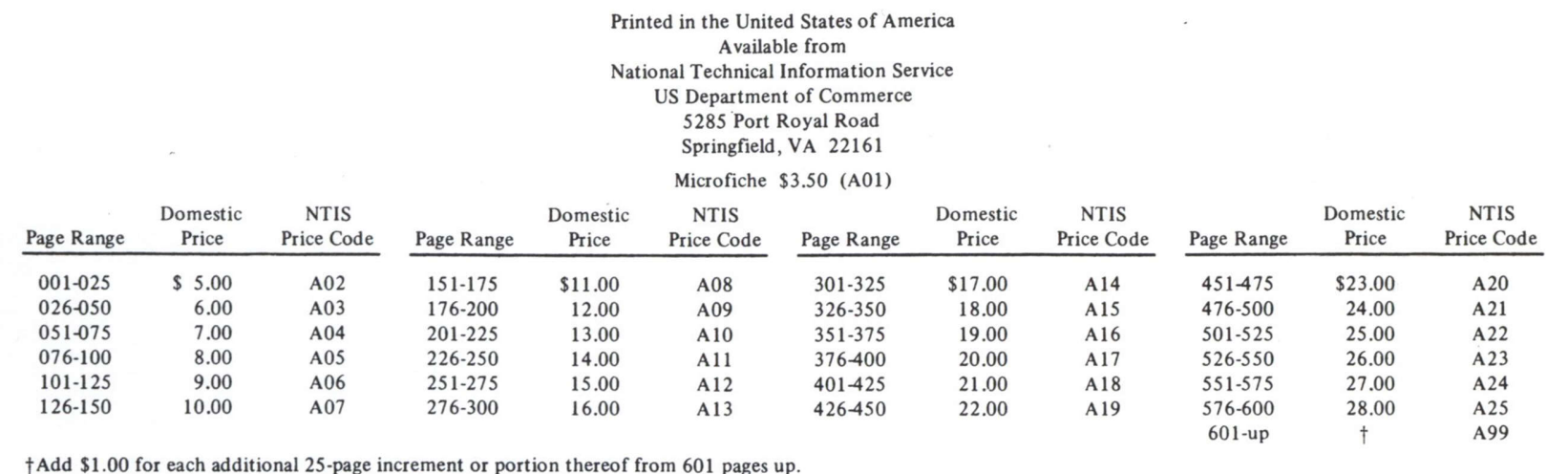


